\title{
THE SUMMER MEETING IN ANN ARBOR
}

The sixtieth Summer Meeting of the American Mathematical Society was held from Tuesday, August 30, through Friday, September 2, 1955, at the University of Michigan, Ann Arbor, Michigan. Several other organizations met in Ann Arbor at about the same time: the Mathematical Association of America, the Institute of Mathematical Statistics, the Econometric Society, the Association for Symbolic Logic, the Society for Industrial and Applied Mathematics, the Industrial Mathematics Society, and Pi Mu Epsilon.

Over 800 people registered for the meeting, including 600 members of the Society. This was a record attendance for a Society meeting.

Professor Nathan Jacobson of Yale University delivered the Colloquium lectures, entitled Jordan algebras, on Tuesday at 11:00 A.M. and Wednesday, Thursday, and Friday at 9:00 A.M. Presiding offiers were Professors R. L. Wilder, A. A. Albert, Marshall Hall, Jr., and T. H. Hildebrandt.

By invitation of the Committee to Select Hour Speakers for Annual and Summer Meetings, Professors Raoul Bott, of the University of Michigan, and Edwin Hewitt, of the University of Washington, addressed the Society. Professor Bott spoke on Some applications of the Morse theory to the topology of manifolds at 1:30 P.M. Tuesday, and Professor Hewitt on Convolution algebras at 11:00 A.M. Wednesday. Presiding officers were Professors G. A. Hedlund and A. D. Wallace, respectively.

There was a joint session of the Society with the Econometric Society on Extremum problems, which was held in two parts on Thursday at 10:15 A.M. and 1:45 P.M. The lecturers were Professors Jacob Wolfowitz, Samuel Karlin, P. C. Rosenbloom, and Leonid Hurwicz. The presiding officers were Professors E. G. Begle and A. W. Tucker.

There was a joint session of the Society with the Industrial Mathematics Society on Friday at 10:15 A.M. There was an address by Dr. Saul Rosen of Wayne University entitled The influence of digital computers on numerical analysis, and four short papers. Professor A. W. Jacobson presided.

There were nineteen sessions for contributed papers. The presiding officers were Professors R. H. Bing, R. H. Bruck, A. H. Copeland, Dr. J. H. Curtiss, Dr. D. A. Flanders, Professors A. M. Gleason, J. W. Green, A. E. Heins, A. W. Jacobson, J. P. LaSalle, C. J. Nesbitt, G. de B. Robinson, H. J. Ryser, A. C. Schaeffer, R. D. Schafer, Seymour Sherman, P. A. Smith, R. M. Thrall, and C. B. Tompkins. 
More papers were read at this meeting than at any other meeting of the Society.

The Committee on Arrangements provided a highly enjoyable program for the entertainment and recreation of the guests of the University. In addition, the running of the business of the meeting was carried on with commendable efficiency. The Associate Secretary takes this opportunity to pay tribute to Professor Wilfred Kaplan, the Chairman of the Committee; the meeting is a monument to his patience and energy.

The Council met on Tuesday evening, August 30, 1955.

The Secretary announced the election of the following forty-one persons to ordinary membership in the Society:

Mr. Abdul Kadir Aziz, University of Maryland;

Mr. Peter Greenough Bartlett, Askania Regulator Co., Chicago, Illinois;

Mr. Errett Albert Bishop, University of California, Berkeley;

Mr. Albert James Brinson, Seton Hall University;

Mr. Clarence Harlan Cook, The Glenn L. Martin Company, Baltimore, Maryland;

Mr. William Edwin Cummins, David Taylor Model Basin, Washington, D. C.;

Mr. Robert Edwin Daniels, Haller, Raymond and Brown, Inc., State College, Pennsylvania;

Mr. John Frank Detlef, Ballistic Research Labs.;

Reverend Edward Dennis Eichman, Epiphany Apostolic College;

Miss Constance Mary Foley, University of New Hampshire;

Dr. Gerald Henry Fraser Gardner, Cornell University;

Dr. Baruch Germansky, Universität Frankfurt, Mainz, Germany;

Mr. Hyman Housman, New York University;

Mr. Bernard Benedict James, Boeing Airplane Co., Seattle, Washington;

Pfc. Floyd Idwal John, United States Army, Dugway, Utah;

Miss June Rose Mary Jensen, Polytechnic Institute of Brooklyn;

Mr. James Kenyon Knowles, Massachusetts Institute of Technology;

Mr. Neophytos Kockinos, University of California, Berkeley;

Mr. Yehiel Lehrer, Weizmann Institute of Science, Israel;

Mr. Ronald Melvin Lewis, University of Southern California;

Mr. Ralph Aloysius Luebbe, University of Cincinnati;

Mr. Jon N. Mangnall, Cornell Aeronautical Laboratory, Buffalo, New York;

Miss Ann Muzyka, Polytechnic Institute of Brooklyn;

Mr. Anthony Joseph Nespole, College of the City of New York;

Mr. Edward Valentine Oborsh, University of Chicago;

Mr. Julius Louis Pallone, Polytechnic Institute of Brooklyn;

Sister Julia Pflum, College of Mount St. Joseph;

Dr. Roger Stanton Pinkham, Duke University;

Mr. David Alexander Pope, University of California, Los Angeles;

Miss Susan Pyeatt, Catholic University of America;

Professor Fazlollah Reza, Massachusetts Institute of Technology;

Dr. Donald Eugene Richmond, Convair, Ft. Worth, Texas;

Mr. Robert Steele Roth, Aberdeen Proving Ground, Maryland;

Dr. Parfeny Pavlovich Saworotnow, Catholic University of America;

Mr. Thomas I. Seidman, AEC Computing Facility, New York; 
Mr. Charles Robert Seliger, Rutgers University;

Mr. James Leroy Snyder, University of Cincinnati;

Mr. Bernard Albert Sobel, Ethyl Corp., Baton Rouge, Louisiana;

Mr. Vytas Francis Volertas, Aircraft Armaments, Inc., Cockeysville, Maryland;

Mr. Edward Joseph Voltaggio, Ballistic Research Laboratories, Aberdeen Proving

Ground, Maryland;

Miss Martha Becket Walton, University of Nancy, Nancy, France.

It was reported that the following eight persons had been elected to membership on nomination of institutional members as indicated:

Brooklyn College: Mr. Abraham Karrass.

University of Cincinnati: Mr. Smbat Abian.

University of Michigan: Mr. Donald Warren Crowe.

Oregon State College: Mr. Robert Lewis Brock, Mr. Robert Wallace Brown, and Mr. Louis Baker Rall.

Santa Clara University: Mr. Samuel A. Francis.

Tulane University: Miss Ruth Evans Biggers.

The Secretary announced that the following had been admitted to the Society in accordance with reciprocity agreements with various mathematical organizations: Deutsche Mathematiker-Vereinigung: Dr. Heinz Bauer, Mathematical Institute, University of Erlangen, Germany; Indian Mathematical Society: Dr. N. Padma, Annamalai University, Annamalainagar, India; Svenska Matematikersamfundet: Professor Karl Gustav Natanael Hössjer, Chalmers University of Technology, Göteborg, Sweden; Unione Matematica Italiana: Mr. Flavio Marani, Banca d'Italia, Roma, Italy.

The following appointments by the President were reported: as Chairman of the Committee on Translations from Russian and Other Foreign Languages for a term of one year beginning July 1, 1955: J. L. Doob; and as members for terms of three years beginning July 1, 1955: R. P. Boas and Irving Kaplansky (Committee now consists of J. L. Doob, Chairman, R. E. Bellman, R. P. Boas, Irving Kaplansky, and Hans Samelson); as Chairman of the Organizing Committee for Summer Institutes for a period of one year beginning July 1, 1955: Edwin Hewitt; and as members for a period of three years beginning July 1, 1955: Nelson Dunford and E. R. Kolchin (Committee now consists of Edwin Hewitt, Chairman, S. S. Chern, Nelson Dunford, F. B. Jones, E. R. Kolchin, and J. J. Stoker; as an ad hoc committee on local arrangements for the 1955 Summer Institute: R. H. Bing, Chairman, R. H. Bruck, R. C. Buck, and A. C. Schaeffer; as auditors for 1955: C. R. Adams and C. H. W. Sedgewick; as a committee on Mathematicians Dismissed for Political Reasons: E. E. Moise, Chairman, W. L. Duren, H. W. Kuhn, R. D. James, and J. B. Rosser; as a committee to study privileges of membership inso- 
far as publications are concerned: A. E. Meder, Jr., Chairman, E. G. Begle, and Einar Hille.

The following appointments to represent the Society were reported: at the 100th anniversary of St. Mary's College and the dedication of St. Mary's Science Hall at Notre Dame, Indiana, on April 23, 1955: Professor A. E. Ross; at the inauguration of President George W. Starcher of the University of North Dakota on April 20, 1955: Professor R. D. Staley; at the inauguration of Arthur B. Bronwell as President of Worcester Polytechnic Institute on April 30, 1955: Professor C. T. Bumer; at the inauguration of Willis McDonald Tate as President of Southern Methodist University on May 5, 1955: Professor C. R. Sherer; at the inauguration of Harold P. Rodes as President of Bradley University on May 6, 1955: Professor Evelyn K. Kinney; at the dedication of the Burlington Nuclear Laboratories on May 23, 1955: Professor J. H. Roberts; at the inauguration of Daryl Chase as President of Utah State Agricultural College on June 3, 1955: Professor C. R. Wylie, Jr.; at the 75th anniversary of the American Society of Mechanical Engineers in Boston, June 19-24, 1955: Professor W. T. Martin; at the dedication of the United States Air Force Academy, Lowry Air Force Base, Denver, Colorado, July 11, 1955: Professor W. J. Thron.

The Secretary reported that Professor Paul Olum had accepted an invitation to deliver an hour address at the meeting to be held at the University of Maryland on October 22, 1955; that Professor P. R. Garabedian will deliver an address at the meeting to be held at the University of Southern California on November 12, 1955; that Professor E. H. Spanier will deliver an address at the meeting to be held at the University of Wisconsin, Milwaukee, on November 25-26, 1955; that Professor Everett Pitcher will address the Society at the meeting to be held at Hunter College on February 25, 1956, and that Professor Fritz John will deliver an address at the Society meeting to be held at Columbia University on April 20-21, 1956.

The Secretary reported that the Agricultural and Mechanical College of Texas had been elected to institutional membership.

The Secretary reported that Professor A. W. Tucker had been elected to complete the term of Professor J. R. Kline on the Committee on Printing and Publishing.

The Council voted to set meetings November 12, 1955, at the University of Southern California; February 25, 1956, at Hunter College, New York City; and April 20-21, 1956, at Columbia University. The Council set the dates of the Annual Meeting in 1956 to be December 27-29. 
The Council voted to grant an annual subvention of $\$ 1000$ a year for three years to a new Illinois Journal of Mathematics.

The Council voted to approve a Summer Research Institute for the summer of 1956 on the topic "Differential Geometry in the Large."

The Council voted to approve a Symposium on Applied Mathematics on the topic "Calculus of Variations and Its Applications," to be held in conjunction with the spring meeting in Chicago in 1956 with the co-sponsorship of the Office of Ordnance Research.

The Council authorized and requested the President to appoint a committee to consider desirable extensions of the Society's activities.

The Council adopted the following resolution:

"It is with a sense of irreparable loss that the Council of the American Mathematical Society records the passing on May 2, 1955, of John Robert Kline, who was Secretary of the Society for ten years and who was a member of this body for all but one of the past twenty-seven years.

"Kline's association with the Society dates from 1915 when he presented as his first paper his Doctoral Dissertation "Double Elliptic Geometry in Terms of Point and Order." Since he did not become a member until the next year, it was necessary that he be "introduced" for this presentation by his teacher, Professor R. L. Moore. Over the next thirteen years the scientific programs of the Society were enriched by some twenty-three papers of his, chiefly on Analytic Topology.

"His official service to the Society seems to have begun in 1925 with membership on a Committee on Summer Meetings. Two years later he became an Associate Editor of the Transactions in which capacity he served for ten years. Soon he was elected to the Council and began twelve years as Associate Editor of the Bulletin and nine years in the same capacity for the American Journal of Mathematics. Later he served for four years on the Editorial Board for the Colloqiuum Publications. He was Associate Secretary from 1933 to 1936 . Among the innumerable committees of which he was a member was a Preliminary Committee on the International Congress of which he became Secretary in September, 1938. This committee began planning to hold a Congress in 1940, but its labors were not to come to fruition until 1950.

"When Dean Richardson retired at the end of 1940, Kline was a natural choice for election as Secretary. He had experience, connections national and international, a love of work which gave him an almost unbelievable capacity for accomplishment, a powerful memory for facts, dates, and people, and thorough familiarity with the status of mathematical activity and personnel in this country. For the next decade he guided the Society's affairs wisely, firmly, gently. For he had those gifts of tact and diplomacy that come only from an innate gentleness of spirit, a spirit unyielding on matters of principle but never giving unnecessary offense.

"In the war years defense needs dominated mathematical activity, while the war's end saw the Society grow with almost alarming speed in numbers, in strength and in the scope of its activities; but Kline met both the variety and the number of demands upon him without wavering. He had a large share in the formation in 1945 of the Policy Committee for Mathematics which he served as Secretary and whose work continued the national emphasis that had arisen in wartime.

"Nor was his influence on the mathematical community due solely to his official acts. Many are the young mathematicians who have profited by his kindly guidance 
in launching their careers; and many are the seasoned department chairmen who have sought his advice on their administrative problems.

"Probably he himself regarded his work as Secretary of the Organizing Committee for the International Congress and then of Congress itself as his most satisfying achievement in mathematical organization. In a sense his entire career had prepared him for this-from his early studies abroad through his manifold administrative experience in university and scientific organizations. Patiently he guided and coordinated the work of the various subcommittees in conqucring the difficulties of passport regulations, travel arrangements, finance, international politics, and the rest. And when the Congress had closed its most successful sessions, he was rightfully entitled to the true satisfaction of a great task well done, as he wound up its affairs and retired from the Secretaryship of the Society-from the Secretaryship but not from the service. In the last five years of his life he served as Trustee, was on several important committees, was a representative on the National Research Council, and was Chairman of the Policy Committee. But his health, which had never been robust though not too many of his associates knew of his physical sufferings, grew steadily worse until his final release from pain.

"To his widow, Mrs. Eunice Story Kline, the Council extends its sympathy and its appreciation of a life of truly devoted service to mathematics and to mathematicians."

(An In Memoriam article on Professor Kline, written by Professor B. P. Gill, will appear in an early issue of the Bulletin.)

A business meeting was held on Wednesday morning, August 31, 1955. Preceding the meeting Professor J. W. Green reported on the Survey of Research Potential and Training in Mathematics and requested the cooperation of all mathematicians in this Survey.

A resolution of thanks to the host institution was adopted.

At the business meeting, the following amendments to the by-laws were approved: To amend Article III, Sections 2, 3, and 4 to read:

Section 2. There shall be seven Editorial Committees as follows: committees for the Bulletin, for the Proceedings, for the Colloquium Publications, for Mathematical Surveys, for Mathematical Reviews, a joint committee for the Transactions and the Memoirs and a committee consisting of the representatives of the Society on the Board of Editors of the American Journal of Mathematics.

Section 3. There shall be a Committee on Printing and Publishing.

Section 4. The size of each Publication Committee shall be determined by the Council.

To amend Article VII, Section 1 to read:

Section 1. The term of office shall be one year in the case of the President Elect and the Ex-President, five years in the case of the Trustees, two years in the case of the President, Vice Presidents, the Secretary, the Associate Secretaries and the Treasurer. The term of office in case of members of the Publications Committees shall be three years, except that when the size of a Publications Committee is 
changed the Council may authorize the election of a member for a term of less than three years.

To add a new Section 9 to Article VII as follows:

Section 9. The following provisions shall govern the election of Trustees in 1955 and 1956. The Trustees in office on January 1, 1955, shall serve until their respective terms expire. At the annual meeting in 1955, one Trustee shall be elected for a term of five years and one for a term of two years. At the annual meeting of 1956, one Trustee shall be elected for a term of five years, one for a term of three years, and one for a term of two years. This section shall be repealed without further action on January 1, 1957.

The abstracts of the papers follow. Those having the letter " $t$ " following the abstract number were read by title. Where a paper, presented in person, has more than one author, the symbol (p) follows the name of the author who presented it. Mr. Block was introduced by Professor A. A. Albert, Mr. Bratton by Professor T. C. Koopmans, Professors Fryer and Halperin by Professor H. W. Ellis, Professor Grothendieck by Professor G. B. Price, Mr. Gutierrez-Burzaco by Professor Ky Fan, Mr. Milnes by Professor G. G. Lorentz, Dr. Pinkham by Professor D. V. Widder, and Professor Wasel by Reverend T. J. Saunders.

\section{Algebra ANd Theory of Numbers}

632. Maurice Auslander (p) and D. A. Buchsbaum: Global dimension of noetherian rings. I.

The authors prove that if a local ring $A$ has finite global dimension, then the Krull dimension of $A$ equals global dimension of $A$. They show that for Krull dimension zero and one, finite global dimension implies $A$ is regular. This leads to the conjecture that a local ring is regular if and only if it has finite global dimension. One consequence of this conjecture is that if $P$ is any prime ideal of the regular local ring $A$, then $A_{P}$ is also regular. A new proof of the Cohen-Macaulay theorem is obtained without the use of structure theorems. It is also shown that if $B$ is a commutative, noetherian ring of finite global dimension, then it is the direct sum of a finite number of integral domains. Thus, if $B$ is also hereditary, then it is the direct sum of a finite number of Dedekind rings. The techniques of homological algebra were employed extensively, in conjunction with standard ideal theory in noetherian rings. (Received July $21,1955$.

\section{3t. E. H. Batho: On noncommutative semi-local and local rings.}

In this paper, the basic structure of a class of rings which reduce in the commutative case to the well-known semi-local and local rings are studied. A semi-local ring $R$ is one having the following properties: (a) $R$ satisfies the maximum condition for left ideals; (b) $R$ modulo its Jacobson radical $J$ satisfies the minimum condition for left ideals; (c) $\bigcap_{i=0}^{\infty} J^{i}=(0)$. $R$ becomes a topological ring with respect to the topology induced by the powers of $J$ and the basic topological and ideal-theoretic properties 
of $R$ and its completion are studied. It is shown that a complete semi-local ring (or the completion of any semi-local ring) is isomorphic to a vector space direct sum of a finite number of full matrix rings over completely primary rings plus a subgroup of $J$ and certain refinements of this decomposition are discussed. It is shown that a complete local ring (or the completion of any local ring) is isomorphic to a full ring of matrices over a completely primary ring and this structure is shown to characterize complete local rings. Certain concordancy conditions are discussed and a modified chain condition for a certain class of ideals is introduced. Some of the above results are applied to integral extensions of commutative local rings (Curtis, Proc. Amer. Math. Soc. vol. 4 (1953) pp. 945-955) and some examples are discussed. (Received July $18,1955$.

\section{I. A. Barnett and Smbat Abian (p): A note on skew-symmetric} elements of a ring.

Let $\left({ }^{\prime}\right)$ denote an involutionary anti-automorphism of a ring $R^{*}$. Let $A, C \in R^{*}$ where $C$ is symmetric such that $A-C$ possesses an inverse $B$. Then $A$ is skew-symmetric if and only if: $2 B^{\prime} C B+B^{\prime}+B=0$. In particular if $\left({ }^{\prime}\right)$ denotes transposition in the ring of finite real $n$ by $n$ matrices and if $a_{i j}+a_{i i}=0$, the preceding relation will imply $\left|a_{i j}-c \delta_{i j}\right|\left(A_{i j}+A_{j i}\right)+2 c \sum_{1}^{n} A_{i r} A_{j r} \equiv 0$ where $c$ is a real number and $\left(A_{i j}\right)$ is the adjoint matrix of the matrix $\left(a_{i j}-c \delta_{i j}\right)$. Again in the algebra of the operators $(a, K)$ over real numbers, consider $(a, K)^{\prime} f(x)=a f(x)+\int_{0}^{1} k(y, x) f(y) d y$, where $k(x, y)$ is the kernel of the operator $(a, K)$. If $b(x, y)$ is the reciprocal of $k(x, y)$, in the sense of Fredholm, then a necessary and sufficient condition that $k(x, y)$ be skew-symmetric is that: $2 \int_{0}^{1} b(s, x) b(s, y) d s+b(y, x)+b(x, y) \equiv 0$. From this follows that the reciprocal of a skew-symmetric kernel is never skew-symmetric. (Received July 18, 1955.)

\section{D. W. Blackett: The near-ring of affine transformations.}

A few familiar facts about the affine transformations of a vector space over a field are: 1 . The nonsingular affine transformations form the "affine group." 2. The translations are a normal subgroup of the affine group. 3. The quotient group of the affine group modulo the translation group is isomorphic to the group of nonsingular linear transformations. Unfamiliar analogues of these facts are: I. The affine transformations (singular ones included) form a near-ring with commutative addition. II. The constant transformations form the unique maximal two-sided ideal of the near-ring of affine transformations. III. The quotient near-ring of affine transformations modulo constant transformations is isomorphic to the simple ring of linear transformations. (Received June 27, 1955.)

\section{Richard Block: Some properties of new simple Lie algebras.} Preliminary report.

Several classes of new simple Lie algebras of characteristic $p$ are examined for the properties of being restricted and having a nondegenerate trace form. The simple algebras $S_{n}(n>2)$ [M. S. Frank, Proc. Nat. Acad. Sci. U.S.A. vol. 40 (1954)] of all truncated derivations of divergence zero of the polynomial algebra $B_{n}=F\left[x_{1}, \cdots, x_{n}\right]$ $\left(x_{i}^{p}=0\right)$ are restricted and any trace form for any of them is identically zero. The simple algebras $V_{m}(n=2 m, p>2)$ of dimension $p^{2 m}-2$ consisting of all derivations $D(\phi)=\left(a_{1}, \cdots, a_{m} ; b_{1}, \cdots, b_{m}\right)$ where $a_{i}=\partial \phi / \partial x_{i+m}, b_{i}=-\partial \phi \partial x_{i}$ and $\phi$ has no term in $\left(x_{1}, \cdots, x_{2 m}\right)^{p-1}$ are restricted and have a nondegenerate trace form defined in terms of the form for the monomials $\phi=x_{1}^{i_{1}} \cdots x_{2 m}^{i_{2 m}}, \psi=x_{1}^{j_{1}} \cdots x_{2 m}^{j_{2 m}}$ by making $\tau(D(\phi), D(\psi))=1$ if $i_{1}+j_{1}=\cdots=i_{2 m}+j_{2 m}=p-1$ and zero otherwise. For $m=1$, these 
are the only trace forms, up to scalar multiples. The simple algebras $T_{n}(n, p>2)$ of all derivations with divergence equal to coordinate sum are not restricted. (Received July 18, 1955.)

637t. J. L. Brenner: Bounds for determinants. Conditions that a matrix of constants be regular.

This paper concerns a condition for the nonsingularity of a matrix $A=\left(a_{i j}\right)$ of complex numbers. The condition reads as follows. Define the $p$ th power average $L_{i, p}$ for the $i$ th row by $L_{i, p}=\left[\sum^{\prime}\left|a_{i j}\right|^{p}\right]^{1 / p}, p^{-1}+q^{-1}=1$. If $S_{p}=\sum\left(1+\left|a_{i i}\right|^{q} L_{i, p}^{-q}\right)^{-1}$ is less than unity $\left(0^{q} 0^{-q}=0\right)$, then $\operatorname{det} A$ is not 0 . (The condition is due to Ostrowski, Univ. Roma Ist. Naz. Alta Mat. Rend. Mat. e Appl. (5) vol. 10 (1951) pp. 156-168; Mathematical Reviews vol. 14, p. 125.) Two new proofs of the validity of this condition are given, and upper and lower bounds for $|\operatorname{det} A|$ are found. From them, a nontrivial lower bound for the absolute value of the determinant of an arbitrary matrix is obtained, which is adapted for use with automatic computers. Credit is due the Office of Ordnance Research for sponsoring this work. (Received July 8, 1955.)

638t. Leonard Carlitz: A degenerate Staudt-Clausen theorem.

Put $x\left\{(1+\lambda x)^{\mu}-1\right\}^{-1}=\sum_{0}^{\infty} \beta_{m}(\lambda) x^{m} / m$ !, where $\lambda \mu+1$, so that $\beta_{m}(\lambda)$ is a polynomial in $\lambda$ with rational coefficients. The following results are proved. 1 . Let $\lambda=a / b$, $(a, b)=1$. Then $\beta_{2 m}(\lambda)=A_{2 m}-\sum p^{-1}$, where $A_{m}$ is a rational number whose denominator contains only primes occurring in $b$, and the summation is over primes $p$ such that $p-1|2 m, p| a$. 2. Let $\lambda$ be integral $(\bmod p), p \nmid a b, p^{r} \mid m$. Then $\beta_{m}(a \lambda / b)$ $\equiv(a / b)^{m} \beta_{m}(\lambda)\left(\bmod p^{r}\right)$. 3. Let $p \geqq 3, p^{r}|m, p| \lambda$. Then $\beta_{m}(\lambda) \equiv 0\left(\bmod p^{r}\right)$ if $p-1 \nmid m$, while $\beta_{m}(\lambda)+p^{-1}-1 \equiv 0\left(\bmod p^{r}\right)$ if $p-1 \mid m$. (Received June 27,1955 .)

\section{9t. Leonard Carlitz: A further note on Dedekind sums.}

Let $\bar{B}_{r}(x)$ denote the Bernoulli function. Put $c_{r, s}(h, k)=\sum_{\mu} \bar{B}_{r}(\mu / k) \bar{B}_{s}(h \mu / k)$ and $f_{m}(h, k ; \tau)=\sum_{s=0}^{m}\left(\begin{array}{c}m \\ s\end{array}\right)(k \tau-h)^{m-8-1} c_{m-s, s}(h, k)$. A transformation formula of Apostol (Duke Math. J. vol. 17 (1950) pp. 147-157 leads to the formula $f_{m}(H, K ; \tau)=(-1)^{m}$ $\cdot(k t-h)^{m-2} f_{m}\left(h_{1}, k_{1}, \tau_{1}\right)+f_{m}(h, k, \tau)$, where $\tau_{1}=\left(h^{\prime} \tau+k^{\prime}\right)(k \tau-h), h h^{\prime}+k k^{\prime}+1=0$, $h_{1} h_{1}^{\prime}+k_{1} k_{1}^{\prime}+1=0$ and $\left(\begin{array}{c}H^{\prime} K^{\prime} \\ K-H\end{array}\right)=\left(\begin{array}{l}h_{1}{ }^{\prime} k_{1}^{\prime} \\ k_{1}-h_{1}\end{array}\right)\left(\begin{array}{l}h^{\prime} k^{\prime} \\ k-k\end{array}\right)$. An elementary proof of this formula is given. It is then shown that for $m=2$ and $\left(k, k_{1}\right)=1$, the formula reduces to a threeterm relation for Dedekind sums recently proved by Rademacher (Duke Math. J. vol. 21 (1954) pp. 391-397). (Received June 27, 1955.)

\section{0t. Leonard Carlitz: An extension of Bauer's congruence.}

Extending the well known congruence of Bauer, the author shows that $Q(z)$ $=\prod_{r_{i}=0}^{p^{m}=1}\left(z+r_{1} u_{1}+\cdots+r_{k} u_{k}\right) \equiv\left\{\Delta\left(u_{1}, \cdots, u_{k}, z\right) / z \Delta\left(u_{1}, \cdots, u_{k}\right)\right\}^{p^{k(m-1)}}\left(\bmod p^{m}\right)$, where in the product $\left(r_{1}, \cdots, r_{k}, p\right)=1$ and $\Delta\left(x_{1}, \cdots, x_{k}\right)$ is the determinant $\left|x_{i}^{p i}\right|(1 \leqq$ $i \leqq k, 0 \leqq j \leqq k-1)$. If $Q(z)=z^{n}+C_{1} z^{n-1}+\cdots+C_{n}$, where $n=p^{k m}-p^{k(m-1)}$, it follows that $C_{r} \equiv 0\left(\bmod p^{m}\right)$ for $\left.p-1\right\} r ;$ moreover $C_{2 r+1} \equiv 0\left(\bmod p^{2 m-1}\right)$ for all $r$ and $C_{2 r+1} \equiv 0$ $\left(\bmod p^{2 m}\right)$ for $p-1+2 r$. For small values of $r$ these results can usually be improved. Put $R_{t}=\sum_{0}^{p^{m-1}}\left(r_{1} u_{1}+\cdots+r_{k} u_{k}\right) t$. Then $R_{t} \equiv 0\left(\bmod p^{m k-k^{\prime}}\right)$ where $k^{\prime}=[k /(p-1)]$. In particular it follows that $C_{t} \equiv 0\left(\bmod p^{\min (m k, m k-k+t)}\right.$ for $1 \leqq t<p-1$. We have also $R_{t} \equiv p^{m k} B_{t}^{(k)}\left[u_{1}, \cdots, u_{k}\right]\left(\bmod p^{m k+m-k^{\prime}}\right)$, where $B_{t}^{(k)}\left[u_{1}, \cdots, u_{k}\right]$ is a Bernoulli number of order $k$ in the notation of Nörlund. (Received June 27, 1955.)

\section{1t. Leonard Carlitz: A sum connected with quadratic residues.}

Let $p=2 k+1$ be a prime, $m \geqq 1, a$ arbitrary. Put $S_{m}(a)=\sum_{r=0}^{m}(-1)^{m-r}$ 
$\cdot((r+a) / p)\left(\begin{array}{r}m \\ r\end{array}\right)$. Define $h$ and $l$ by means of $(h-1)(p-1)<m-(p-1) / 2 \leqq h(p-1)$, $m+l=h(p-1)+(p-1) / 2$. Then $S_{m}(a)$ is divisible by $p^{h}$ at least; also the residue $(\bmod p)$ of $p^{-h} S_{m}(a)$ is obtained. In particular $p^{-h} S_{m}(a) \equiv-(-1)^{k} / k !(\bmod p)$ for $l=0, p^{-h} S_{m}(0) \equiv-(-1)^{k} m / 2 \cdot k !(\bmod p)$ for $l=1$, etc. (Received June 27,1955 .)

\section{2t. Leonard Carlitz: The coefficients of $\sinh x / \sin x$.}

Put $\sinh x / \sin x=\sum_{0}^{\infty} \beta_{2 m} x^{2 m} /(2 m)$ !, so that the $\beta$ 's are rational numbers. In seeking a theorem of the Staudt-Clausen type for $\beta_{2 m}$ the following result was obtained. The denominator of $\beta_{2 m}$ contains only primes $p \equiv 3(\bmod 4)$. Let $p^{r} \mid(2 m+1)$, $p^{r+1} \nmid(2 m+1)$ and define $u, v$ by means of $2 m+1 \equiv u+v p\left(\bmod p^{2}-1\right), 0 \leqq u \leqq p-1$; $0 \leqq v \leqq p-1$. Then $\left.(2 m+1) \beta_{2 m} \equiv 1-\sum(-1)^{s}{ }_{s(p-1)}^{s M+1}\right)\left(p^{-1}-1\right)\left(\bmod p^{r}\right), 0<s(p-1)$ $<2 m+1$. If $u+v \geqq p$ the denominator of $\beta_{2 m}$ is divisible by exactly $p^{r}$; if $u+v<p$ the denominator of $\beta_{2 m}$ is divisible by $p^{r}$ at most. Like results hold for the coefficients in the expansion of $\left(e^{\omega x}-1\right) /\left(e^{x}-1\right)$, where $\omega$ is a primitive $n$th root of unity. (Received June 27, 1955.)

\section{3t. Eckford Cohen: A class of arithmetical functions. II. Applica-} tions.

In Part I of this paper (Bull. Amer. Math. Soc. Abstract 61-4-446) it was shown that a function $f(n)=f(n, r)$ of period $r \geqq 1$ is even $(\bmod r)$ if and only if it has a Fourier expansion, $f(n, r)=\sum_{d \mid r} \alpha(d) c(n, d), c(n, d)=\sum_{(x, d)=1} \exp (2 \pi i n x / d)$, or equivalently, an arithmetical representation, $f(n, r)=\sum_{d \mid(n, r)} g(d, r / d)$. In this paper the theory of Part I is applied to several functions. (1) The number of solutions $N(n, r, t)$ of the congruence $n \equiv x_{1} y_{1}+\cdots+x_{t} y_{t}(\bmod r)$ is deduced, furnishing a unified treatment, in a particular case, of a congruence problem considered previously (Bull. Amer. Math. Soc. Abstracts 61-3-388, 389). (2) Carmichael's sum, $c^{(8)}(n, r)=\sum_{d \mid(n, r)} d^{s} \mu(r / d)$, is represented in two different forms, from which the Ramanujan and Hölder evaluations of $c(n, r)$ are shown to result. (3) Completely even functions $(\bmod r)$, defined by the property $f(n, r)=h((n, r))$, are introduced, and the cases $h(m)=\phi_{s}(m) / m^{s}$, $h(m)=\sigma_{s}(m), s$ arbitrary, are studied. (4) As corollaries, a number of arithmetical identities are derived, some of which are known while others appear to be new. (Received July 15,1955 .)

\section{4t. C. W. Curtis: On commuting rings of endomorphisms.}

A simple abstract method of proof is given for the results of Weyl (Duke Math. J. vol. 3 (1937) pp. 200-212) on the invariant subspaces relative to the centralizer $C$ of a finite group $G$ of semi-linear transformations in a finite dimensional vector space $M$ over a field $K$. The method applies to the more general situation obtained by replacing $K$ by an arbitrary division ring $\Delta$, and by removing the hypotheses that $M$ is finite dimensional over $\Delta$, and that $M$ is completely reducible relative to $G$. The result states that there exists a (1-1) correspondence, preserving direct sums, intersections, and isomorphism relations, between the $C$-direct summands of $M$ and those right ideal direct components of the semi-linear group ring $A$ of $G$ which are contained in a certain two-sided ideal $A_{0}$ in $A$. If $M$ is finite dimensional over $\Delta$, then every indecomposable $C$-direct summand $R$ of $M$ possesses a unique maximal submodule $S$, and two indecomposable $C$-direct summands $R_{1}$ and $R_{2}$ are $C$-isomorphic if and only if $R_{1} / S_{1}$ and $R_{2} / S_{2}$ are $C$-isomorphic. By means of a natural duality in the theory, it is also shown how the structure of $M$ as a $G$-module determines the ideal theoretic structure of the centralizer $C$. (Received July 14, 1955.) 
645. C. W. Curtis: On the structure and representation theory of Lie algebras of characteristic $p>0$.

Let $L$ be a semi-simple Lie algebra over the field of all algebraic numbers, and let $H$ be a Cartan subalgebra of $L$. Then $L$ has a basis $\left(X_{i}\right)$, called an admissible basis of $L$ relative to $H$, with certain special properties. With $\left(X_{i}\right)$ is associated a number field $K$ containing the constants of structure determined by the basis $\left(X_{i}\right)$. A set of prime ideals in $K$ is defined, which contains almost all prime ideals in $K$. For a fixed prime ideal $P$ in the set, let $O$ be the valuation ring, and $\bar{K}$ the residue field. Then $\Sigma=\Sigma O X_{i}$ is a Lie ring, and $\bar{L}=\Sigma / P \Sigma$ is a Lie algebra over $\bar{K}$ with nondegenerate Killing form. The natural mapping $T: \Sigma \rightarrow \bar{L}$ maps $H$ onto a Cartan subalgebra of $\bar{L}$, and sets up a (1-1) correspondence between the root diagram of $L$ relative to $H$ and the root diagram of $\bar{L}$ relative to $H T$. In a natural way, $\bar{L}$ becomes a restricted Lie algebra in the sense of Jacobson. A theory of weights, corresponding to the CartanWeyl theory in the classical case, is developed for restricted representations of $\bar{L}$. Every irreducible representation $U$ of $L$ determines a restricted representation $\bar{U}$ of $\bar{L}$, and various properties of the correspondence $U \rightarrow \bar{U}$ are established. (Received July $14,1955$.

646. R. A. Dean: Completely free lattices generated by partially ordered sets.

Let $P$ be a partially ordered set with elements $p_{i}$ and order relation ( $\left.\leqq\right)$, and let the set, $C F(P)$, of lattice polynomials (words) be ordered by: (i) $p_{i} \leqq p_{j}$ if and only if $p_{i} \leqq p_{i}$ in $P$, and, (ii) recursively, $A \leqq B$ if and only if one or more of the following hold: (1) $A \equiv A_{1} \cup A_{2}$ and $A_{1}$ and $A_{2} \leqq B_{1}$, (2) $A \equiv A_{1} \cap A_{2}$ and $A_{1}$ or $A_{2} \leqq B$, (3), (4), the dual conditions. Identifying words $A$ and $B$ when $A \leqq B$ and $B \leqq A, C F(P)$ becomes the completely free lattice generated by $P$. The word problem is solved in $C F(P)$ and $C F(P)$ can be mapped homomorphically into any lattice in which $P$ may be embedded. Results analogous to those obtained for the free lattice on unordered elements by Whitman (Ann. of Math. vol. 42 (1941) pp. 325-330 and vol. 43 (1942) pp. 104-115) show the existence of canonical forms and give criteria for completely free sublattices. In general $C F(P)$ preserves neither least upper nor greatest lower bounds existing in $P$, but in special cases $C F(P)$ is isomorphic to the free lattice, $F L(P)$, generated by $P$ and preserving those bounds of pairs of elements. In particular it is shown that when $P=[a, b, c, d ; a>b, c>d]$ and $Q=[a, b, c, d, e ; a>b>c>d$, e], $C F(P)(\cong F L(P))$ contains infinite chains and is a sublattice of $C F(Q)(\cong F L(Q))$. (Received June 23, 1955.)

\section{7t. G. D. Findlay and Joachim Lambek: Calculus of bimodules.}

In addition to the ordinary tensor product of two bimodules $A$ and $B$ it is natural to define two tensor quotients: $A$ over $B$ and $A$ into $B$. The former is written $A / B$ and means Hom $(B, A)$, where $B$ and $A$ are regarded as right modules, turned into a bimodule in the natural way. For "associative laws" involving the three tensor operations are then valid, for example $(A / B) / C \cong A /(C \cdot B)$. Four others hold conditionally, thus $A \cdot(B / C) \cong(A \cdot B) / C$ if $A$ or $C$ is direct summand of a right module with finite base. This condition on $A$ can also be expressed by saying that there exists an identity matrix over $A$ regarded as a right module. By the matrix ring over the right module $A$ is understood the tensor product of $A$ with its dual, turned into a ring in the natural way. The calculus of bimodules is applied to the extension of operator rings. In fact, the remaining eight associative laws depend on a generalization of 
Frobenius extensions. Modules are not assumed to be unitary. (Received June 30, 1955.)

\section{Malcolm Goldman: Subalgebras of certain Banach algebras.}

In Annals of Mathematics (2) vol. 53 (1951) pp. 235-249 Kaplansky defines the concept of an $\mathrm{AW}^{*}$-algebra and the concept of type for such algebras. In American Journal of Mathematics vol. 75 (1953) pp. 839-858, he proves that an $\mathrm{AW}^{*}$-algebra $B$ of type $I$ is the algebra of all continuous endomorphisms of a certain kind of module. Using this spatial theory and methods of Kaplansky and von Neumann, the author proves: (1) an isomorphism between two maximal abelian (self-adjoint) subalgebras of $B$ which leaves the center fixed can be extended to a unitary element of $B$; (2) if $A$ is a self-adjoint subalgebra of $B$ which is a double commutant and of type $J$ (I, II, or III), then the commutant of $A$ is of type $J$. (Received July 18, 1955.)

649. Franklin Haimo: Component preserving automorphisms of semidirect products. Preliminary report.

Let $G$ be a semi-direct product of a normal subgroup $H$ by a subgroup $K$. It is proved that those automorphisms of $G$ which do not alter the components in $H$ of each element of $G$ form a subgroup of the group of automorphisms of $G$, and that this subgroup is itself a semi-direct product of an abelian group by a group of automorphisms of $K$. Let $A$ be an automorphism of $G$ which carries each element of $G$ onto an image with the product of the components in $H$ of element and image equal to the identity. Then $A$ carries the centralizer of $H$ in $G$ onto a (direct) product $H L$ where $L$ is that subgroup of $K$ such that the inner automorphisms of $G$ generated by the elements of $L$ are precisely those inner automorphisms of $G$ generated by elements of $K$ which reduce to the identity on $H$. Likewise $A$ carries $H L$ onto the centralizer of $H$ in $G$. See W. H. Mills, Trans. Amer. Math. Soc. vol. 74 (1953) pp. 428-443, where, in the case of the holomorph of $H, A(H)$, the conjoint of $H$, is this centralizer. This work has been supported in part by the OSR, USAF. (Received July 18, 1955.)

\section{0t. Bruno Harris: Centralizers of simple Jordan algebras. Pre- liminary report.}

Let $A$ be an involutorial ring $L(M, M)$ (all linear transformations on the vector space $M$ over a division ring $D$ having adjoints relative to a nondegenerate scalar product in $M$ ). Let $J$ be the Jordan ring of self-adjoint elements of $A, K$ a Jordan subring of $J$ containing $F_{1} \cdot 1, F_{1}$ being the self-adjoint elements of the center $F$ of $D$, and $J(K)$ the linear transformations in $J$ commuting with every linear transformation in $K$. Theorem: Let char. $D \neq 2$. Let $K$ be simple finite-dimensional over $F_{1} \cdot 1$, $B$ its enveloping associative ring. Assume (a) $B \supseteq F \cdot 1$, (b) dimension of $M$ over $D>2$-dimension over $F$ of a minimal right ideal of $B$. Then: 1 . If $B$ is simple, $J(K)$ is the set of self-adjoint elements of an involutorial ring $L(N, N)$. 2. If $B$ is not simple, there exist a pair of dual spaces $N_{1}, N_{2}$ such that $J(K)$ is the set of self-adjoint elements of $L\left(N_{1}, N_{2}\right) \oplus L\left(N_{2}, N_{1}\right)$ relative to the involution mapping elements of $L\left(N_{i}, N_{j}\right)$ on their adjoints in $L\left(N_{i}, N_{i}\right)$. Also, unless $K$ is of type $D$ (i.e. space of elements of degree $\leqq 1$ of a Clifford algebra), $J(J(K))=K$. (Received July 18,1955.)

651. Melvin Henriksen: Concerning adequate rings and elementary divisor rings. III. 
A commutative ring $S$ with unit is called an elementary divisor ring if, for every matrix $M$ over $S$, there exist nonsingular matrices $K, L$ such that $K M L$ is diagonal. $S$ is adequate if (i) all finitely generated ideals are principal and (ii) for every $a, b \in S$, $a \neq 0$, there exist $r, s \in S$ such that $a=r s,(b, r)=S$, and for every non-unit divisor $t$ of $s,(t, b) \neq S$. The following improvements of results announced in Bull. Amer. Math. Soc. Abstract 61-2-182 have been obtained: 1. If $S$ is adequate, every proper prime ideal of $S$ is contained in a unique maximal ideal. 2. There exist integral domains that are elementary divisor rings, but are not adequate. 3. If $S$ satisfies (i) and each element of $S$ outside its Perlis-Jacobsen radical is in at most a finite number of maximal ideals, then $S$ is an elementary divisor ring (but need not be adequate). (The author was supported by the National Science Foundation, contract no. NSFG1129.) (Received July 18, 1955.)

652t. J. H. Hodges: An exponential sum for symmetric matrices in a finite field. Preliminary report.

For $\alpha \in G F(q), q$ odd, let $e(\alpha)=e^{2 \pi i t(\alpha) / p}$, where $t(\alpha)=\alpha+\alpha^{p}+\cdots+\alpha^{p^{n-1}}$. If $A=\left(\alpha_{i j}\right)$ is a square matrix with elements in $G F(q)$, let $\sigma(A)=\sum \alpha_{i i}$. Consider the sum $S=S(B, \Lambda, A)=\sum e\left\{\sigma\left(\Lambda^{\prime} X+X^{\prime} \Lambda\right)\right\}$, where $A$ is a nonsingular symmetric matrix of order $m, B$ is a symmetric matrix of order $t, \Lambda$ is an arbitrary $m \times t$ matrix, and the sum is over all $m \times t$ matrices $X$ satisfying the equation $X^{\prime} A X=B$, all matrices having elements in $G F(q)$. If $\Lambda=0, S$ is the number of solutions $X$ of $X^{\prime} A X=B$, which is given in (L. Carlitz, Representations by quadratic forms in a finite field, Duke Math. J. vol. 21 (1954) pp. 123-128). In Weighted quadratic partitions over a finite field (Canadian J. of Mathematics vol. 5 (1953) pp. 317-323) L. Carlitz has considered $S$ for $t=1$. Explicit values for this $S$ are given in all except one case and in all cases $S$ is given in terms of certain Kloosterman sums over $G F(q)$. In the present paper it is shown that the general sum $S$ can be expressed in terms of Kloosterman sums defined for symmetric matrices over $G F(q)$. A number of the properties of these Kloosterman sums are also given. (Received July 15, 1955.)

653. R. E. Johnson: Structure theory of faithful rings. II. Restricted rings.

A ring $R$ is (left) faithful if $a R \neq 0$ for every nonzero $a \in R$. Designate by $\mathcal{L}(R)$ the lattice of right ideals of $R$ and by $\mathcal{F}(R)$ the set of all ideals $A$ of $R$ for which $A \cap A^{l}=0$ and $A=A^{l l}$, where $A^{l}$ is the left annihilator of $A$. The set $\mathcal{F}(R)$ is shown to be a Boolean algebra if the union operation $\bigcup$ is defined by: $A \bigcup_{B}=(A+B)^{l l}$. If $\mathcal{F}(R)=\{0, R\}, R$ is called irreducible. Associated with $R$ is a unique maximal extension ring $\bar{R}$ that contains $R$ as an ideal and is such that $\bar{R}^{l}=0$ in $\bar{R}$. The Boolean algebras $\mathcal{F}(R)$ and $\mathcal{F}(\bar{R})$ are shown to be isomorphic. The ring $R$ is called restricted if $\mathcal{F}(R)$ is a complete Boolean algebra. A closure operation $f$ is induced on $\mathcal{L}(R)$ by $\mathcal{F}(R)$ if $R$ is restricted. If $f$ is atomic and if the union $B$ of the atoms of $\mathcal{F}(R)$ is such that $B^{l}=0$, then there exists a set $\left\{A_{i}\right\}$ of irreducible rings such that $\sum_{i} A_{i} \subseteq R$ $\subseteq \sum_{i}^{*} \bar{A}_{i}$, where $\sum\left(\sum^{*}\right)$ designates the discrete (full) direct sum. An analysis is made of the structure of a right $R$-module $M$ induced by an atomic closure operation on $\mathcal{L}(R)$. The extended centralizer of $R$ over $M$ is a full direct sum of primitive rings. (Received June 8, 1955.)

\section{Irving Kaplansky: Rings resembling operator algebras.}

An $A W^{*}$-algebra can be defined as a $C^{*}$-algebra $A$ in which every annihilator is 
of the form $e A$ with $e$ an idempotent. The latter condition is meaningful in any ring, and in this paper its consequences are studied. In due course it becomes advisable to postulate an involution and add two axioms concerning projections and square roots. The theory can be pushed to the point just before one introduces a trace. Some of the proofs are shorter than the old ones. (Received July 29, 1955.)

\section{J. H. B. Kemperman: Complexes in arbitrary groups.}

Consider two finite nonempty subsets $A$ and $B$ of a multiplicative group $G$ and let $C=A B$ be the set of all the elements $c=a b$ with $a \subset A, b \subset B$. Let $k=[A]+[B]$ $-[A B]$, where $[D]$ denotes the number of elements in $D$. Let $c_{0}=a_{0} b_{0}\left(a_{0} \subset A, b_{0} \subset B\right)$ be a fixed element of $C$. Conjecture: $\left(^{*}\right) c_{0}$ admits at least $k$ different representations of the form $c_{0}=a b$ with $a \subset A, b \subset B$. This is trivially true when $k \leqq 1$. It is shown that: (1) Assertion $\left({ }^{*}\right)$ holds when $[B] \leqq k+2$, hence, when $[B] \leqq 4$. (2) Let $b_{0}, b_{1}, \cdots, b_{p}$ $(p \geqq k-1)$ be the set of all those elements $b$ in $B$ for which $a_{0} b b_{0}^{-1} \subset A$. Then $\left(^{*}\right)$ holds when $b_{i} b_{0}^{-1} b_{j}=b_{j} b_{0}^{-1} b_{i}(i, j=1, \cdots, p)$. Thus $p=1$ implies $\left(^{*}\right)$ (also $p=2$ and $[B] \leqq k+3 \leqq 6)$. (3) From (2), $\left(^{*}\right)$ holds for each element $c_{0}$ in $C$ when the elements of $B$ commute with each other, hence, when $G$ is abelian. An equivalent form of conjecture $\left({ }^{*}\right)$ is: $\left(^{* *}\right)$ When some element $c_{0}$ of $A B$ admits at most $k_{0}$ representations $c_{0}=a b$, then $[A B] \geqq[A]+[B]-k_{0}$. From (3), $\left({ }^{*}\right)$ holds when $G$ is abelian. For $k_{0}=1$, this was shown by L. Moser and P. Scherk, Amer. Math. Monthly vol. 62 (1955) p. 46. (Received July 18, 1955.)

\section{L. A. Kokoris: On a class of almost alternative algebras.}

A finite dimensional algebra $\mathfrak{A}$ over a field $\mathfrak{F}$ is called an algebra of $(\gamma, \delta)$-type if it satisfies the identities $z(x y)=(z x) y+\gamma(x z) y-\gamma x(z y)+\delta(y z) x-\delta y(z x)$ and $(x y) z$ $=x(y z)+\gamma(x z) y-\gamma x(z y)+(\delta-1)(y z) x-(\delta-1) y(z x)$ where $\gamma$ and $\delta$ are elements of $\mathfrak{F}$ satisfying $\gamma^{2}-\delta^{2}+\delta=1$. These algebras are a type of almost alternative algebras which arise in the study of almost alternative algebras relative to quasi-equivalence. When $\mathfrak{F}$ has characteristic $\neq 2,3,5, \mathfrak{A}$ is power-associative. If also $\delta \neq 0,1$ and $\mathfrak{A}$ has an idempotent $e, \mathfrak{A}$ may be decomposed relative to $e$ in the same way as an associative algebra. The radical of $\mathfrak{A}$ is defined to be the maximal nilideal of $\mathfrak{A}$ and it is then proved that a simple algebra is either associative or has a unity quantity which is absolutely primitive. A semisimple algebra is shown to be a direct sum of simple algebras. (Received July 13, 1955.)

657t. Walter Ledermann and B. H. Neumann: On the order of the automorphism group of a finite group. I.

A function $f(n)$ is found with the property that every finite group whose order is greater than or equal to $f(n)$ has at least $n$ automorphisms. The least such bound satisfies $f(1)=1, f(2)=3, f(3)=f(4)=7$, and $f(n) \leqq(n-1)^{n+(n-2)\left[\log _{2}(n-1)\right]}$ when $n>4$. (Received July $7,1955$. )

658t. C. S. Lin and H. B. Mann: A generalization of a theorem of Cauchy. Preliminary report.

Let $G$ be a cyclic group of prime power order, $A, B$ sets of elements in $G$. Put $C=A \oplus B=\{a \oplus b ; a \in A, b \in B\}$, where $\oplus$ denotes the addition in $G$. Let $H$ be the largest subgroup of $G$ for which $A \oplus B \oplus H=A \oplus B$ and let $(A)$ denote the number of elements in $A$. Then $(C)+(H) \geqq(A)+(B)$. The corresponding theorem for sets of 
nonnegative integers is not true. The methods are those introduced by H. B. Mann in several previous papers. (Received May 27, 1955.)

\section{9t. R. C. Lyndon: A nonspecial operator Lie ring.}

A Lie ring $L$, over a commutative ring $R$ of operators, is constructed, which is not $R$-isomorphic to a Lie subring of any associative $R$-ring $A$. (The present example seems to be of a quite different nature from that given by Shchirshchov, Usp. Mat. Nauk vol. 8 (1953) pp. 173-175.) $R$ is the ring of polynomials over the integers in indeterminates $\xi_{1}, \xi_{2}, \xi_{3} . L$ has generators $x_{1}, x_{2}, x_{3}$, and defining relations $\xi_{i} x_{j}=\xi_{j} x_{i}$. Let $u=\sum \xi_{i}\left[x_{j}, x_{k}\right]$, summed cyclically. Then $u \neq 0$ in $L$ (although $2 u=0$ ), while $u$ vanishes under any $R$-homomorphism of $L$ into associative $A$. (Received July 19, 1955.)

660t. P. J. McCarthy: Sufficient conditions for a genus of indefinite ternary quadratic forms to contain only one class. II.

Let $f$ be a ternary quadratic form with integral matrix $A$. Let $\Omega$ be the g.c.d. of the two-rowed minor determinants of $A$, and let $\Delta$ be the integer determined by $|A|=\Omega^{2} \Delta$. Let $F$ be the reciprocal form of $f$, i.e., the form with matrix $\Omega^{-1}|A| A^{I}$. The genus of $f$ contains only one class if the following conditions are satisfied: $\Omega \equiv 4$ $(\bmod 8)$ and $\Delta$ is odd, $(\Omega, \Delta)=1,(-1)^{(f-1) / 2}=(-1)^{(\Delta-1) / 2}$, and the congruence $F \equiv-\Omega / 4(\bmod 4)$ is solvable. This extends a theorem of A. Meyer (see L. E. Dickson, Studies in the theory of numbers, Chapter 4). (Received July 13, 1955.)

\section{P. J. McCarthy: Witt's cancellation theorem in valuation rings.}

Let $R$ be a valuation ring in which 2 is a unit. It is not assumed that $R$ is complete. Two $n$-ary quadratic forms over $R$ are said to be congruent in $R$ if there is a unimodular transformation (i.e., a transformation whose determinant is a unit in $R$ ) taking one of the forms into the other. A short and quite simple proof is given of the following theorem: if $f\left(x_{1}, \cdots, x_{r}\right)+g\left(x_{r+1}, \cdots, x_{n}\right)$ and $f\left(x_{1}, \cdots, x_{r}\right)+h\left(x_{r+1}\right.$, $\left.\cdots, x_{n}\right)$ are congruent in $R$, then $g$ and $h$ are congruent in $R$. This generalizes the cancellation theorem of Witt (J. für Math. vol. 176). It has been proved by Durfee with the added assumption that $R$ is complete (Duke Math. J. vol. 11). (Received July 13, 1955.)

662. T. S. Motzkin and E. G. Straus (p): Some combinatorial extremum problems.

For a class of functions $F\left(x_{1}, \cdots, x_{n}\right)$ including Gauss brackets (denominators of continued fractions) of positive integers and lengths of routes through certain point sets in metric spaces the arrangements of the given $n$ numbers which maximize and minimize $F$ are $\cdots a_{5} a_{3} a_{1} a_{2} a_{4} \cdots$ and $a_{1} a_{n} a_{3} a_{n-2} \cdots a_{4} a_{n-1} a_{2}$ where $a_{1} \geqq a_{2}$ $\geqq \cdots \geqq a_{n}$. Related results are obtained for cyclic and infinite problems. (Received June 10, 1955.)

\section{L. J. Paige: A class of simple Moufang loops.}

Let $R$ be a simple alternative, not-associative ring possessing an idempotent not its unit element. Let $L$ be the loop of all regular elements of $R$ and let $Z$ be the center of $L$. Then either $L / Z$ is a simple, not-associative Moufang loop or $L / Z$ contains a simple, not-associative Moufang subloop. This result yields finite simple Moufang loops whose possible orders are $\left(2^{7 n}-2^{3 n}\right)$ or $2^{-1}\left(p^{7 n}-p^{3 n}\right)$ if $p$ is an odd prime. The 
corresponding result for the Cayley-Dickson division algebra over the real field $R^{*}$ is valid but is not true for all fields. (Received July $5,1955$. )

664. Irving Reiner: Integral representations of cyclic groups of prime order.

Let $G$ be a cyclic group of prime order $p$. It is shown here that $G$ has exactly $1+2 h_{p}$ inequivalent indecomposable rational integral representations, where $h_{p}$ is the class number of the cyclotomic field of $p$ th roots of unity over the rationals. In matrix terminology, this shows that every $n \times n$ integral matrix solution of $X^{p}=I$ is integrally similar to a direct sum of matrices chosen from a certain set of $1+2 h_{p}$ such solutions. This generalizes results of Latimer and MacDuffee (Ann. of Math. vol. 34 (1933) pp. 313-316) for the case $n=p-1$, and of Hua and Reiner (Trans. Amer. Math. Soc. vol. 71 (1951) pp. 331-348) for the case $p=2$. The proof depends upon (i) the work of Latimer and MacDuffee (op. cit.) on classes of nonderogatory matrices with given characteristic polynomial, (ii) Zassenhaus' results (Abh. Math. Sem. Hansischen Univ. vol. 12 (1938) pp. 276-288) connecting rational and integral equivalence of representations, and (iii) a generalization of Maschke's theorem due to Gaschütz (Math. Zeit. vol. 56 (1952) pp. 376-387). (Received April 18, 1955.)

665t. Irving Reiner and J. D. Swift: Congruence subgroups of matrix groups.

Let $\mathcal{D}$ be the ring of integers in an algebraic number field and $G$ the group of $2 \times 2$ matrices over $\mathcal{D}$ with determinant +1 . Let German letters denote ideals in $\mathcal{D}$ and define $G(\mathfrak{N})$ to be the subgroup of all matrices $\left(a_{i j}\right) \in G$ for which $a_{21} \in \Re$. It is shown that if $H$ is a group satisfying $G(\mathfrak{M N}) \subset H \subset G(\mathfrak{R})$ where $(\mathfrak{M}$, (6)) $=(1)$, then $H=G(\mathfrak{D N})$ for some $\mathfrak{D} \supset \mathfrak{M}$. This generalizes a theorem of M. Newman (Duke Math. J. vol. 22 (1955) pp. 25-32) for the case where $\mathcal{D}$ is the ring of rational integers. Examples are given to show that the restriction $(\mathfrak{M},(6))=(1)$ is not superfluous. A second generalization of Newman's theorem is obtained by considering matrices of higher order. Let $M_{r}^{+}$denote the modular group consisting of all integral $r \times r$ matrices with determinant +1 . Define groups $C_{m}=\left\{\left(a_{i j}\right) \in M_{r}^{+} ; m \mid a_{i 1}\right.$ for $\left.i>1\right\}$, and $R_{n}=\left\{\left(a_{i j}\right) \in M_{r}^{+}\right.$; $n \mid a_{r j}$ for $\left.j<r\right\}$. It is proved that if $H$ is a group satisfying $\left(C_{a m} \cap R_{b n}\right) \subset H \subset\left(C_{m} \cap R_{n}\right)$, where $(a m, b n)=1$, then $H=C_{\alpha m} \cap R_{\beta n}$ with $\alpha|a, \beta| b$. (Received May 25, 1955.)

\section{D. W. Robinson: Continuity of a matric function.}

Let $T$ be an element of the space of finite square matrices over the field of complex numbers. Let $f$ be a complex valued function of a complex variable, defined in a neighborhood of each of the eigenvalues of $T$ and analytic at the eigenvalues of index greater than one. Then using the classical definition of a matrix function and the ordinary notion of a limit of a matrix, the following theorem is proved: The matric function $f$ is continuous at $T$ if and only if $f$ is continuous at the eigenvalues of $T$ and analytic at each repeated eigenvalue of $T$. (Received July 15, 1955.)

667t. Alex Rosenberg and Daniel Zelinsky: Cohomology into the ring of all linear transformations. Preliminary report.

Let $C$ be the algebra of all linear transformations on a vector space $V$ over a division algebra $K$. Then $\left(^{*}\right) H^{n}(C, C)=H^{n}(K, K)$. When $n=1$, this is a theorem on derivations which will appear in a forthcoming book by N. Jacobson. In the course of proving $\left({ }^{*}\right)$ we also show that if $B$ is any subalgebra of $C$ then $\left({ }^{*}\right) H^{n}(B, C)$ 
$=\mathrm{Ext}_{B}^{n} \otimes_{K}(V, V)$. In case $n=1,(* *)$ yields two more theorems in Jacobson's book. First, if the base field is the center of $K$ and $B$ is simple of finite order, then $H^{1}(B, C)$ $=0$. Secondly, if $B$ is any primitive ring with nonzero socle $S$ and $V$ is a left $B$-module with $S V=V$, then every derivation of $B$ into the ring of endomorphisms of the group $V$ is inner $\left[\left(^{* *}\right)\right.$ is used after extending $B$ to be an algebra over a prime field $\left.K\right]$. Both $\left({ }^{*}\right)$ and $\left({ }^{* *}\right)$ are direct consequences of identities to be found in Homological algebra by H. Cartan and S. Eilenberg. (Received July 18, 1955.)

668t. Alex Rosenberg and Daniel Zelinsky: Cohomology of infinite algebras. II.

If $A$ is an algebra over a field $F, F$ - $\operatorname{dim} A$ is the supremum of the integers $n$ for which there is a two-sided $A$-module $N$ with $H^{n}(A, N) \neq 0$. If the field $K$ is a subalgebra of the center of $A$, then $F$-dim $K \leqq F-\operatorname{dim} A \leqq F-\operatorname{dim} K+K$-dim $A$. If $A$ is a field of transcendence degree $t$ over $F$ and $K$ is generated by a transcendence basis of $A$, the inequality yields $t \leqq F-\operatorname{dim} A$. When $t<\infty, t=F-\operatorname{dim} A$ if and only if $A$ is finitely, separably generated over $F$. The case $t=1$ was already reported in (Bull. Amer. Math. Soc. Abstract 61-2-207). If $A$ has no separable generation, then $F$-dim $A$ $=\infty$. In proving these results, it is also shown that if $A$ is a union of a countable tower of simple algebras of finite orders, then $H^{1}(A, A \otimes A) \neq 0$. (Received July $18,1955$.

\section{Alex Rosenberg (p) and Daniel Zelinsky: Dimension of matrix rings.}

H. Cartan and S. Eilenberg have defined the left global dimension of a ring $R$ as the supremum of the projective dimensions of left $R$-modules. We show that if $M$ is the ring of $n$ by $n$ matrices over $R$ and $T$ is the ring of $n$ by $n$ triangular matrices over $R$, then 1. gl. dim. $M=1$. gl. $\operatorname{dim} . R$ and 1 . gl. dim. $T=1+1$. gl. dim. $R$. Furthermore, if $R$ is an algebra over a commutative ring $F$, then $F$-dim $M=F$-dim $R$ and $F$-dim $T=1+F$-dim $R$. When 1. gl. $\operatorname{dim}$. $R=0$ or $F$-dim $R=0$ the results for $T$ have already been proved by Eilenberg, Nagao, and Nakayama. (Received July 18, 1955.)

\section{0t. M. F. Ruchte: Rings of quotients.}

A ring $S$ is said to be a (right) quotient extension of a ring $R$ if $S \supseteq R$ and if, for nonzero $s$ in $S, s R \cap R \neq 0$. R. E. Johnson [Bull. Amer. Math. Soc. vol. 60 (1954) p. 96] conjectures that the maximal elements of the set of all quotient extensions of $R$, ordered by inclusion, are isomorphic and that quotient extensions of $S$, where $S$ is a quotient extension of $R$, are quotient extensions of $R$. A proof is given for both of these statements based upon Eckmann and Schopf [Arch. Math. vol. 4 (1953) pp. 75-78]. (Received July 18, 1955.)

\section{1t. H. J. Ryser: Maximal determinants in combinatorial investiga- tions.}

Let $Q$ be a 0,1 matrix of order $v$, containing exactly $t 1$ 's. Let $k$ denote a positive real, and set $\lambda=k(k-1) /(v-1)$. If $t \leqq k v$ and $0<\lambda \leqq k-\lambda$ or if $t \geqq k v$ and $0<k-\lambda \leqq \lambda$, then $|\operatorname{det} Q| \leqq k(k-\lambda)^{(v-1) / 2}$. In the preceding theorem, if $|\operatorname{det} Q|=k(k-\lambda)^{(v-1) / 2}$, then $Q$ is the incidence matrix of a $v, k, \lambda$ configuration (see $\mathrm{S}$. Chowla and $\mathrm{H}$. J. Ryser, Combinatorial problems, Canadian Journal of Mathematics vol. 2 (1950) pp. 93-99). Thus if $Q$ is a 0,1 matrix of order $v$ containing exactly $t 1$ 's, and if $k=t / v$, with $\lambda=k(k-1) /(v-1), 0<\lambda<k<v$, then $|\operatorname{det} Q| \leqq k(k-\lambda)^{(v-1) / 2}$, equality holding if and 
only if $Q$ is the incidence matrix of a $v, k, \lambda$ configuration. (Received May 31,1955.)

672. R. L. San Soucie: Flexible rings.

A. A. Albert (Trans. Amer. Math. Soc. vol. 64 (1948) pp. 552-593) has called a (not necessarily associative) ring flexible in case $(x, y, x)=0$, all $x, y \in R$. For a flexible ring $R$, define $S=[s \in R \mid(s, x, y)=(y, x, s)=0]$, and $N=[n \in R \mid(n, x)=(x, n)=0]$. The flexible identity is studied, using the sets $S$ and $N$. The theorems proved include: (1) A flexible division ring has a unit element if and only if $S \neq 0$. (2) If $R$ is not associative and has no proper divisors of zero, then $R$ is commutative if and only if $(R, R) C S$. (3) If $x^{2}-x \in N$, all $x$ in $R$, then $R$ is commutative. Call a flexible ring $R$ strongly flexible if and only if $x y \cdot z x=x(y z \cdot x)$ and observe that all alternative rings are strongly flexible (R. H. Bruck and Erwin Kleinfeld, Proc. Amer. Math. Soc. vol. 2 (1951) pp. 878-890). Representative theorems include: (1) Let $p$ be zero or any prime greater than one. There exists a finite-dimensional strongly flexible algebra of characteristic $p$ which is not alternative. (2) Every strongly flexible division ring is alternative, and hence has a unit element. (3) Let $R$ be an arbitrary ring having a unit element and characteristic not two. Then $R$ is alternative if and only if $R$ is strongly flexible. (4) Let $R$ be a strongly flexible ring of characteristic zero and embed $R$ in the ring $S$ with unit element in the usual way. Then $S$ is strongly flexible if and only if $R$ is alternative. (5) Let $R$ be a ring of characteristic not two and all nilpotent elements in $N$. Suppose further that $R$ has a left unit $e$. Then $R$ is alternative if and only if $R$ is strongly flexible. (Received July 18, 1955.)

\section{3t. E. V. Schenkman: $A$ direct proof of a theorem of Gruenberg.}

If $G$ is an arbitrary group, then a sequence of commutators and an ordering in the sequence is defined so that every element of $G$ is an ordered sum of terms of the sequence. This leads to a direct proof of the fact (cf. Gruenberg, Proc. Cambridge Philos. Soc. vol. 49 (1953) pp. 377-380) that a finitely generated solvable Engel group is nilpotent and enables one also to give a bound for the class of nilpotency in terms of the number of generators, the length of the derived series, and the order of the Engel condition. (Received July 5, 1955.)

674t. E. V. Schenkman: The principal ideal theorem for some infinitely generated groups.

$G$ will stand for a group, $G^{\prime}$ for its commutator subgroup, and $G^{*}$ for the intersection of the members $G^{m}$ of the descending central series of $G$. We then prove the following three facts. LEMMA 1 . Let $G$ be a p-group with no elements of infinite height; let $G / G^{*}$ be finite and let $G^{*}$ be Abelian. Then $G^{*}$ consists only of the identity and $G$ is finite. LEMMA 2. Let $G$ be a group with no elements of infinite order; let $G / G^{*}$ be finite and let $G^{*}$ be Abelian. If the elements of infinite height of $G$ have order prime to that of $G / G^{*}$, then there is a finite nilpotent subgroup $H$ of $G$ such that $H$ intersects $G^{*}$ only in the identity and such that $H$ and $G^{*}$ generate $G$. THEOREM. If $G / G^{\prime}$ is finite then the transfer of $G$ into $G^{\prime}$ is contained in the second derived group $G^{\prime \prime}$ of $G$ provided that $G$ has no elements of infinite order and that the elements of infinite height have orders prime to the order of $G / G^{\prime}$. (Received June 2,1955.)

675. D. S. Scott: $A$ new characterization of $\alpha$-representable Boolean algebras.

C.-C. Chang characterized those $\alpha$-complete Boolean algebras ( $\alpha-B A$ 's) that are 
$\alpha$-representable ( $\alpha$-RBA's), i.e., $\alpha$-homomorphic images of $\alpha$-complete fields of sets (Bull. Amer. Math. Soc. Abstract 61-4-579). The author has proved: (*) an $\alpha-B A$ is an $\alpha-R B A$ iff every proper principal ideal is included in a prime ideal preserving any $\alpha$-system of $\alpha$-termed sums given in advance. This follows directly from four lemmas: $\left(\mathrm{I}_{\alpha}\right) \alpha-R B A$ 's are characterizable by algebraic equations, which may involve $\alpha$-termed sums. ( $\left(\mathrm{II}_{\alpha}\right.$ ) Every two-element $B A$ is an $\alpha-R B A$. ( III $_{\alpha}$ ) Every algebraic equation holding in two-element $B A$ 's holds in all $\alpha$-RBA's. (IV $\alpha$ ) In an $\alpha$-field of sets every proper $\alpha$-complete ideal is included in a prime ideal preserving any $\alpha$-system of $\alpha$-termed sums given in advance. $\left(I_{\alpha}\right)$ is proved either by Birkhoff's theorem or by a simple transformation of Chang's condition to an explicit equational characterization. Contrast $\left(\mathrm{I}_{\alpha}\right)-\left(\mathrm{III}_{\alpha}\right)$ with $\left(\mathrm{I}_{\sigma}\right)-\left(\mathrm{III}_{\sigma}\right)$ of A. Tarski (Bull. Amer. Math. Soc. Abstract 51-6-677) noticing, however, that not every $\alpha-B A$ is an $\alpha-R B A$ for $\alpha \geqq 2 \mathbf{N}_{0}$. Compare (*) with the known fact that an $\alpha-B A$ is isomorphic to an $\alpha$-field of sets iff every proper principal ideal is included in a prime ideal preserving all $\alpha$-termed sums. (Received July $11,1955$. )

676. Ernst Snapper: Higher-dimensional field theory. II. Linear systems.

Let $E / F$ be a finitely generated field extension, i.e., $E$ and $F$ are commutative fields and $E=F\left(a_{1}, \cdots, a_{n}\right)$, where $a_{1}, \cdots, a_{n}$ belong to $E$. The present paper is the second of a series of three articles which have as purpose to develop an intrinsic theory for $E / F$, by giving a purely field-theoretic treatment of the theory of linear systems of algebraic varieties. Let $L$ denote a finitely generated module of $E / F$. See the author's paper Higher-dimensional field theory, I, Bull. Amer. Math. Soc. Abstract 61-4-458, for the notion of the integral closure $|L|_{i}$ of $L$ and its properties. In the present paper, the author introduces the notion of a projective class of modules of $E / F$, which is the field-theoretic substitute for a projective model of $E / F$. Precisely, when $L \neq 0$, its class $C$ consists of all the modules $(1 / a) L$, where $a \in L$ and $a \neq 0$. In terms of $C$, he defines the notions of divisors of the first kind, zeros and poles of elements of $E$, linear equivalence, linear systems, complete linear systems, etc. The theory of linear systems which arises in this way leans heavily on the properties of $|L|_{i}$. (Received July 18, 1955.)

677. Alfred Tarski: Metamathematical proofs of some representation theorems for Boolean algebras.

Consider the following weaker form of Stone's representation theorem for Boolean algebras ( $B A$ 's) : every $B A$ is a homomorphic image of a field of sets. This statement is directly derivable from three elementary lemmas: (I) The class $K$ of homomorphic images of fields of sets is closed under the formation of homomorphic images, subalgebras, and direct products (and hence, by Birkhoff's theorem, is characterizable by algebraic equations). (II) $K$ contains all two-element $B A$ 's. (III) Every algebraic equation holding identically in two-element $B A$ 's holds in all $B A$ 's. Consider now Loomis' theorem: every $\sigma$-complete $B A$ is a $\sigma$-homomorphic image of a $\sigma$-field of sets. This follows from lemmas $\left(\mathrm{I}_{\sigma}\right)-\left(\mathrm{III}_{\sigma}\right)$ exactly analogous to (I)-(III); $\sigma$-complete BA's are regarded here as $B A$ 's with an additional infinite operation, $\sigma$-addition, hence homomorphisms and subalgebras in (I) are replaced by $\sigma$-homomorphisms and $\sigma$-subalgebras, and equations in $\left(\mathrm{I}_{\sigma}\right)$ and $\left(\mathrm{III}_{\sigma}\right)$ may involve $\sigma$-sums. The proofs of $\left(\mathrm{I}_{\sigma}\right)$ and $\left(\mathrm{II}_{\sigma}\right)$ are elementary. ( $\left.\mathrm{III}_{\sigma}\right)$ was obtained by $\mathrm{D}$. Scott as a direct consequence of Rasiowa-Sikorski's lemma by which every proper principal ideal is included in a prime ideal preserving any denumerable system of infinite sums given in advance. 
As opposed to (III) the proof of $\left(\mathrm{III}_{\sigma}\right)$ involves the axiom of choice. (Received July 11, 1955.)

678t. Bernard Vinograde: A decomposition of the product of inseparable fields.

From the uniqueness of the composite $L M$ of pure inseparable algebraic extensions $L, M$ of finite degree over $K$ (see, for instance, G. Pickert, Inseparable Korpererweiterungen, Math. Zeit. vol. 52 (1949) p. 96) it follows that $L$ and $M$ possess a unique maximal pair of isomorphic subfields. Denote each by $D$. Then $L x_{K} M \cong L x_{D} M+N_{0}$, where $N_{0}$ is an ideal in the radical. In terms of the invariants of the composite $L M$ (Pickert, loc. cit. p. 97) the summand $L x_{D} M$ is a field if and only if $m^{\prime}=m$, while $K=D$ if and only if $\bar{q}_{1}=\cdots=\bar{q}_{m^{\prime}}=1$. (Received July 21,1955 .)

679. E. A. Walker: The solution of Kaplansky's test problem III. Preliminary report.

In Kaplansky's book Infinite Abelian groups, p. 13, the following question for Abelian groups, called test problem III, is asked: if $F$ is finitely generated and $F \oplus G$ is isomorphic to $F \oplus H$, are $G$ and $H$ isomorphic? This paper answers this question in the affirmative and in fact shows that if $F$ is torsion, commutativity of $G$ and $H$ is not needed. It suffices to answer the question for the cases $F$ infinite cyclic and $F$ cyclic of prime power order. These cases are treated separately and only elementary group theory methods are used. If $F, G$, and $H$ are modules over a principal ideal ring the answer is still in the affirmative and the proof is almost identical to the one for groups. (Received June 24, 1955.)

\section{0t. A. L. Whiteman: On the cyclotomic numbers of order sixteen.}

Let $g$ be a primitive root of a prime $p$ of the form $16 f+1$. Let $(h, k)$ denote the number of values of $y, 1 \leqq y \leqq p-2$, for which $y \equiv g^{16 s+h}, 1+y \equiv g^{16 t+k}(\bmod p)$, where the values of $s$ and $t$ are each selected from the integers $0,1, \cdots, f-1$. Emma Lehmer [Canadian Journal of Mathematics vol. 6 (1954) pp. 449-454] has raised the question whether or not $256(h, k)$ is expressible (at least for some $h, k$ ) as a linear combination with integer coefficients of $p, x, y, a$ and $b$, where $p=x^{2}+4 y^{2}=a^{2}+2 b^{2}$, and $a \equiv x \equiv 1(\bmod 4)$. In this note the following four formulas are obtained. If the integer $m$ is defined by means of the congruence $g^{m} \equiv 2(\bmod p)$, then (1) $256(00)$ $=p-47-18 x(f$ even, $m \equiv 4(\bmod 8))$, (2) $256(80)=p-15-18 x-32 a(f$ even, $m \equiv 4(\bmod 8))$, (3) $256(00)=p-31-18 x-16 a(f$ odd, $m \equiv 0(\bmod 8))$, (4) $256(40)$ $=p-15-2 x+16 a(f$ odd,$m \equiv 4(\bmod 8))$. The following theorem is also proved. If 2 is an octic residue of $p$, then the set of 16 th power residues modulo $p$ cannot form a difference set. (Received July 12, 1955.)

\section{L. R. Wilcox: Modular extensions of semi-modular lattices.}

Let $(M, \cup, \cap)$ be a complemented modular lattice. A subset $L$ of $M$ is a joinregular subsystem of $M$ if (1) $0,1 \in L$; (2) if $a, b \in L$, then $a \cup b \in L$; (3) every $a, b \in L$ possess a g.1.b. with respect to elements of $L$; (4) if $a, b \in M, c \in L$ and $a<b<c$, there exists $x \in L$ such that $x \cup b=c, x \cap b=a$. Necessary and sufficient conditions on a lattice $L$ of length $\geqq 6$ that it be isomorphic to a join-regular subsystem of some $M$ are found. These include conditions of semi-modularity, complementation, fullness (i.e., if $b<a \cup b$ and $b$ is not covered by $a \cup_{b}$, then $x$ exists with $b<x<a \cup_{b}$ such that $(a, x)$ is a modular pair), and certain conditions governing the behavior of non- 
modular pairs of hyperplanes if such are present. When $M$ exists, it is unique up to isomorphisms. $M$ is constructed by means of quasi-dual-ideals (subsets $S$ of $L$ satisfying (a) $x \in S, y \geqq x$ implies $y \in S$ and (b) if $z=x \bigcap y$ with $x, y \in S,(x, y)$ a modular pair, then $z \in S$ ) which are generated in a certain sense by one or two elements. The present theory generalizes the well known addition of ideal elements in geometry. (Received July 15, 1955.)

\section{Y. K. Wong: On the solution of linear inequalities.}

A simple proof of Minkowski's theorem on the finiteness of a base for all solutions of a finite system of linear inequalities is given. The method is based on the concept of E. H. Moore's general reciprocals of rectangular matrices (which is related to von Neumann's theory of regular rings). A fundamental property of homogeneous linear inequalities is stated as follows: Let $A$ be a matrix of $m$ rows and $n$ columns with elements in an ordered field, and $R$ its general reciprocal. If the columns of $A$ are linearly independent, then $A x=b$ has a nonnegative solution if and only if $R b \geqq 0$, and $x=R b$ is the unique nonnegative solution. Theorems on the extreme supports of a finite system of points in terms of Moore's general reciprocals are obtained. The results are then applied to the theory of games. (Received July 18, 1955.)

\section{F. B. Wright: Structure of topological abelian groups.}

Let $G$ be a topological abelian group, written additively, $M$ an open semigroup in $G$ not containing 0 and maximal with respect to these properties. Let $b(M)$ denote the complement of the union of $M$ and $-M$. (1) Then $b(M)$ is a closed subgroup of $G$. Define $T$ to be the intersection of all such subgroups $b(M)$ (with $T=G$ if there are none). The closed subgroup $T$ is called the torsion subgroup of $G$. (2) If $G$ is discrete, $T$ consists precisely of the elements of finite order. (3) If $G$ is compact, then $G=T$. (4) If $G$ is connected, there is a continuous homomorphism of $G$ into a real linear topological space having $T$ as kernel. (5) For the additive group of a real linear topological space, the torsion subgroup consists of precisely those elements annihilated by every continuous linear functional. (6) Elementary topological and algebraic proofs are given for most of the structure theory of locally compact abelian groups. (Received July 19, 1955.)

\section{AnALysis}

\section{M. I. Aissen: Summable sets and $L^{*}$-spaces.}

The Weierstrass development of the real number system is imitated to develop the idea of absolute convergence of series, independently of the concept of sequential limit. Then the concepts of limit of a sequence and convergence of a series are introduced, using the notion of absolute convergence. (Received July 18, 1955.)

685. E. J. Akutowicz: A qualitative characterization of Blaschke products in a half-plane.

Any holomorphic function $f(w)$ in $|w|<1$ satisfying the conditions (i) $|f(w)|<1$, (ii) $\lim _{r \rightarrow 1-}(1 / 2 \pi) \int_{0}^{2 \pi} \ln \left|f\left(r e^{i \theta}\right)\right| d \theta=0$ is necessarily of the form $f(w)=e^{i c b}(w), c$ a real number and $b(w)$ the Blaschke product in the unit circle formed with the zeros of $f(w)$. Consider the upper half-plane $\operatorname{Im} z=\operatorname{Im}(x+i y)=y>0$ and prove: Theorem I. Let $B(z)$ be a Blaschke product in the upper half-plane. Then $\int_{-\infty}^{\infty} \ln |B(z)| /\left(1+x^{2}\right) d x \rightarrow 0$ as $y \rightarrow 0+$. Theorem II. Let $F(z)$ be holomorphic and bounded for $y>0$, and such that (1) $\int_{-\infty}^{\infty} \ln |F(z)| /\left(1+x^{2}\right) d x \rightarrow 0$ as $y \rightarrow 0+$. Then $F(z)=M \cdot(\exp (i k z+i c)) B(z)$, where 
$c$ and $k$ are real, $k \geqq 0$, and $B(z)$ is the Blaschke product with the zeros of $F(z)$ as its set of zeros. This uniqueness result is less sharp than the one stated above for the unit circle in that the factor exp (ikz) can occur. This difference originates from the fact that (1) does not correspond to (ii). (Received April 21, 1955.)

686. R. A. Askey (p) and I. I. Hirschman, Jr.: Weighted quadratic norms and ultraspherical polynomials.

Let $p_{n}^{\lambda}(x)(0<\lambda<\infty)$ be the normalized ultraspherical polynomials; let $N_{\alpha, \beta}[f]$ be defined as $\left[\int_{-1}^{1}|f(x)|^{2}\left(1-x^{2}\right)^{\lambda-1 / 2}(1-x)^{\alpha}(1+x)^{\beta} d x\right]^{1 / 2}$; and let $a_{n}=\int_{-1}^{1} f(x)\left(1-x^{2}\right)^{\lambda-1 / 2}$ - $p_{\mathrm{n}}^{\lambda}(x) d x$ so that $f(x) \sim \sum_{0}^{\infty} a_{n} p_{n}^{\lambda}(x)$. Consider the multiplier transformation $T f(x)$ $\sim \sum_{0}^{\infty} t_{n} a_{n} p_{n}^{\lambda}(x)$. It is shown that if $\left|t_{n}\right| \leqq C(n=0,1, \cdots)$ and $\sum_{2_{2}^{n}}^{2^{n+1}}\left|t_{n+1}-t_{n}\right| \leqq C$ $(n=0,1, \cdots)$, then $N_{\alpha, \beta}[T f] \leqq A(\alpha, \beta) C N_{\alpha, \beta}[f](-1 / 2<\alpha, \beta<1 / 2)$. (Received June 24, 1955.)

687. Frederick Bagemihl and Leonard Gillman (p): Some cofinality theorems on ordered sets.

Theorem 1. Let $\phi>0, \rho<\omega_{\alpha}, \beta=\omega_{\alpha} \phi+\rho$; then there exists $M C W(\beta)$, with $|M|$ $=\aleph_{\alpha}$ but $|M \cap W(\gamma)|<\aleph_{\alpha}$ for all $\gamma<\beta$, iff $\rho=0$ and either $\phi$ is isolated or $c f(\phi)$ $=c f(\alpha)$. Let $T(\alpha, \beta)$ denote the lexicographically ordered set of all $\omega_{\beta}$-sequences $t=\left(\tau_{\xi}\right)$, where each $\tau_{\xi}<\omega_{\alpha}$, all but finitely many are 0 , but not all are 0 ; denote by $R(t)$ the set of all elements $>t$. Theorem 2. If $\beta \leqq c f(\alpha)$, then $\beta=c f(\alpha)$ iff there exists $M C T(\alpha, \beta)$, with $|M|=\aleph_{\alpha}$, but $|M \cap R(t)|<\aleph_{\alpha}$ for all $t \in T(\alpha, \beta)$. Theorem 3. If $\beta<c f(\alpha)$ and $M C T(\alpha, \beta)$, with $|M|=\aleph_{\alpha}$, then $M$ contains $\omega_{\alpha}$, and $T(\alpha, \beta)$ has $\exp \aleph_{\alpha}$ subsets similar to $M$. Call $M \boldsymbol{N}_{\alpha}$-homogeneous if every interval has power $\boldsymbol{\aleph}_{\alpha}$. Theorem 4 . For $c f(\alpha)<\alpha$, if $M$ is $\aleph_{\alpha}$-homogeneous and every interval contains both $\omega_{c f(\alpha)}$ and $\omega_{\text {of }(\alpha)}^{*}$, then there exists $E \subset M$, with $|E|=\aleph_{\alpha}$, that contains neither $\omega_{\alpha}$ nor $\omega_{\alpha}^{*}$. Corollary. If $\alpha>0$, then $c f(\alpha)>0$ iff there exists $\alpha_{\alpha}$-homogeneous $M$ such that every $E C M$, with $|E|=\aleph_{\alpha}$, contains $\omega_{\alpha}$ or $\omega_{\alpha}^{*}$. (Received August 29, 1955.)

688. R. B. Barrar: Some estimates for solutions of linear parabolic equations.

This paper treats the Dirichlet type boundary value problem for linear parabolic equations in $n$ dimensions. The method used is patterned after that developed for the study of elliptic equations by J. Schauder [ ̈̈ber lineare elliptische Differentialgleichungen zweiter Ordnung, Math. Zeit. vol. 38 (1934) pp. 257-282]. Estimates for solutions and extensions of present existence theorems are given. (Received July 7, 1955.)

689. J. H. Barrett: Boundedness of solutions of systems of second order differential equations.

Let $\alpha(x)$ be a nontrivial (column) vector solution of the vector-matrix differential equation (I) $\left(P(x) \alpha^{\prime}\right)^{\prime}+Q(x) \alpha=0$, where $P(x)$ and $Q(x)$ are square matrices of real continuous scalar functions on $x \geqq a$ and for each $x \geqq a, P(x)$ is nonsingular. If $W(x)$ is a nonsingular matrix of differentiable scalar functions on $x \geqq a$ such that $W^{*} W^{\prime}$ is negative semi-definite (for example, a constant matrix) then $\|\alpha(x)\| \leqq k$ exp $\{(1 / 2)$ $\left.\cdot \int_{a}^{x}\|G(t)\| d t\right\}$ where $G \equiv(W P)^{*-1}-W Q$ and the $\left(^{*}\right)$ denotes the transpose of the matrix. Furthermore, if $Q P^{*}$ is symmetric, positive definite and differentiable, and its derivative $\left(Q P^{*}\right)^{\prime}$ is positive semi-definite; then there exists a nonsingular differentiable matrix $W(x)$ which satisfies $G \equiv 0$ and such that $W^{*} W^{\prime}$ is negative semi-definite. 
Under the above conditions on $Q$ and $P$ every solution of equation (I) is bounded as $x \rightarrow \infty$. These results are matrix analogs of scalar results of the author (Proc. Amer. Math. Soc. vol. 6 (1955) pp. 247-251). (Received July 15, 1955.)

\section{J. W. Brace: Almost uniform convergence.}

The paper defines almost uniform convergence on an abstract set for a net of real or complex valued functions. The concept is used to investigate topologies on linear spaces. The weak topology (not the weak* topology) on the adjoint space of a locally convex space is determined to be the topology of almost uniform convergence on the bounded sets of the original space. Along with the corresponding dual proposition for the weak topology on the original space, it is shown that the weak* topology on an adjoint space is the topology of almost uniform convergence on the weakly compact sets of the original space. The results are used to investigate the continuity of linear transformations. The notion of almost uniform convergence for sequences of functions can be found in the works of Sirvent and Kantorovitch. (Received July 18, 1955.)

\section{1t. J. W. Brace: The structure of the second adjoint space.}

The paper presents a short proof of the fact that the second adjoint space of a locally convex linear topological space is the union of the closures of the bounded subsets of the original space when the closure operation is performed in the second adjoint space under the topology of uniform convergence on the finite point sets of the first adjoint space. The first known approach to such a theorem was made by Gelfand [see Gantmacher and Smulian, C. R. Acad. Sci. URSS. vol. 17 (1937) pp. 91-94]. For the special case of a Banach space proofs have been given by M. M. Day, Duke Math. J. vol. 8 (1941) pp. 763-770, and H. H. Goldstine, Duke Math. J. vol. 4 (1938) pp. 125-131. The proof of the theorem makes extensive use of polars in moving from one space to its adjoint and conversely. (Received July 18, 1955.)

692. L. R. Bragg: The solutions of a linear ordinary differential equation of the third order about a turning point of the second order.

A study is made of the solutions, for large values of the complex parameter $\lambda$, of the differential equation $y^{\prime \prime \prime}(z)+\lambda h_{1}(z, \lambda) y^{\prime \prime}(z)+\lambda^{2} h_{2}(z, \lambda) y^{\prime}(z)+\lambda^{3} h_{3}(z, \lambda) y(z)=0$, where the $h_{i}(z, \lambda)$ are series of the form $\sum_{j=0}^{\infty} h_{i j}(z) / \lambda_{j}$ with the $h_{i j}(z)$ analytic functions of $z$, in a neighborhood $\Theta_{z}$ of a turning point of the second order-that is, a point at which the discriminant of $\gamma^{3}(z)+h_{10}(z) \gamma^{2}(z)+h_{20}(z) \gamma(z)+h_{30}(z)=0$ has a zero of the second order. The author develops a method for obtaining expansions formally satisfying this equation and shows that these expansions are true solutions in a certain asymptotic sense. The method developed is similar to that developed by R. E. Langer in the case of a turning point of the first order, but differs markedly in a number of places since the role taken by the Bessel function is now taken by the confluent hypergeometric function. The author uses several results of R. W. McKelvey's paper on the solutions of a second order differential equation with a second order turning point. (Received July 15, 1955.)

\section{Donald Bratton: The duality theorem in linear programming.}

We suppose given two ordered locally convex spaces $E, F$ and a continuous linear transformation $T$ between them, and two points $a \in E^{\prime}, b \in F$. One can then define a convex set $K$ in $E$ (resp. $K^{\prime}$ in $F^{\prime}$ ) and a corresponding "program" I (resp. II) of maximizing (resp. minimizing) the functional $a$ (resp. $b$ ) in $K$ (resp. $K^{\prime}$ ), and (III) a third program of minimax for the function $\langle x, a\rangle+\langle b, y\rangle-\langle T x, y\rangle$ in $E_{+} \times F_{+}$. The 
duality theorem states an intimate connection between these three programs (a result similar to Lagrange multipliers), and is known to hold under finite-dimensionality. In the general case, by consideration of the graph of $T$, the present writer reverts the theorem to the situation of just one space $E$ and replaces $T$ by a closed subspace $S$ of $E$. The corresponding results are stated and proved under the hypothesis that $S$ is of type $\mathcal{E}$, i.e., that each continuous positive linear functional in $S$ can be extended as such to all of $E$. Finally, a study of the subspaces of type $\mathcal{E}$ is made. A dual notion, "type $Q$," is introduced, and it is shown that each closed subspace of $E$ is of type $\mathcal{E}$ if and only if each closed subspace is of type $Q$. (Received June 22,1955.)

\section{C. C. Braunschweiger: A geometric construction of L-spaces.}

An $L$-space (cf. S. Kakutani, Ann. of Math. vol. 42 (1941) pp. 523-537) is a Banach lattice whose norm and ordering satisfy the property: $x \geqq \theta, y \geqq \theta$ imply $\|x+y\|=\|x\|+\|y\|$. Denote the convex hull of a set $A$ in a linear space $X$ by the symbol $\langle A\rangle$. A subspace $Y$ of the linear space $X$ is constructed as follows: For a subset $B$ of $X$ (called the "base set" of the construction) let $F=\langle B\rangle, S=\langle F \cup(-F)\rangle$, $Y=\{s S: a \geqq 0\}$, and let $p(x)$ be the Minkowski functional for $S$ on $Y$. Conditions on $B$ and $X$ sufficient to insure that $Y^{\prime}$, the Cantor completion of $Y$, is an $L$-space are considered. For example, if $B$ is a (finitely) linearly independent subset of the linear space $X$ then $Y^{\prime}$ is an $L$-space. In particular the construction is used to prove that a Banach lattice $X$ is homeomorphic to an $L$-space under a linear order-preserving homeomorphism if and only if there exists in $X$ a hyperplane whose intersection with the positive cone is a nonvoid bounded set not containing $\theta$. (Recieved July 8, 1955.)

695t. F. E. Browder: Regularity properties of solutions of elliptic differential equations. I.

Let $A=\sum_{|\alpha|,|\beta| \leqq m}(-1)^{|\beta|} D^{\beta}\left(a_{\alpha \beta}(x) D^{\alpha}\right)$ be an elliptic differential operator of order $2 m$ on the smoothly bounded domain $G$ of $E^{n}$. $\left(\alpha=\left(\alpha_{1}, \cdots, \alpha_{r}\right),|\alpha|=r\right.$, $D^{\alpha}=\partial^{r} / \partial x_{\alpha_{1}} \cdots \partial x_{\alpha_{r}}$. By a weak solution of the equation $A u=f$ is meant a function $u$ having $m$-strong derivatives in $L^{1}$ on every compact subdomain of $G$ and satisfying $\sum_{\alpha, \beta}\left(a_{\alpha \beta}(x) D^{\alpha} u, D^{\beta} \phi\right)=(f, \phi)$ for $\phi \in C_{c}^{\infty}(G)$. It is shown that if $u$ is a weak solution of an elliptic boundary-value problem in the variational sense on $G$, and if $a \in C^{m+t}(\bar{G})$, $t \geqq 0$, then $u$ has $2 m+t$ strong derivatives in $L^{2}(G)$ and there exist constants $c_{t} \geqq 0$ independent of $u$ such that $\left\|D^{2 m+t} u\right\|_{L^{2}(G) \leqq} \leqq c_{t}\left\{\left\|D^{t f}\right\|_{L^{2}(G)}+\|u\|_{L^{1}(G)}\right\}$. It follows from a theorem of Sobolev that for $t>n / 2-2 m, u$ will have $2 m+t-n / 2$ continuous derivatives on the closure of $G$ if the $t$ th derivatives of $f$ lie in $L^{2}(G)$. In particular, the derivatives of $u$ involved in the boundary-value problem will exist and satisfy the boundary conditions in the classical sense. The proof utilizes a special case of an inequality due to Aronszajn on coercive differential forms. (Received July 19, 1955.)

696t. F. E. Browder: Regularity properties of solutions of elliptic differential equations. II.

Let $u$ be a weak solution of the equation $A u=f$ of $I$ on the arbitrary domain $G$ of $E^{n}$. Suppose $a \in C^{t}(G), t \geqq 0$. Then there exist constants $c_{t}\left(G_{1}\right)$ independent of $u$ for compact subdomains $G_{1}$ of $G$ such that $u$ has $m+t$ strong derivatives in $L^{p}\left(G_{1}\right)$ satisfying $\left\|D^{m+t} u\right\|_{L^{p}\left(G_{1}\right)} \leqq c_{t}\left(G_{1}\right)\left\{\|f\|_{L^{s}(G)}+\|u\|_{L^{1}(G)}\right\} .(s=n p /(n+p)(m-t))$ if $t \leqq m$, $p$ if $t \geqq m$.) For $a \in C^{0}(G)$, the existence of a fundamental solution in the small and Green's functions for variational boundary value problems follow, while for $a \in$ $C^{m+1}(G)$ it is shown that every weak solution must be a differentiable solution in the classical sense for $f \in L^{p}(G), p>n / 2 m+1$, and the $2 m$ th derivatives will satisfy a 
Holder condition. Finally for regular solutions of $A u=\sum_{|\gamma| \leqq 2 m} c_{\gamma} D^{\gamma} u=f$, the $2 m-1$ st derivatives will satisfy a Holder condition if $g \in C^{0}(G), p>n / 2 m$, while the $2 m$ th derivatives will satisfy a Holder condition if $c_{\gamma}$ and $f$ do. The proofs rest upon the trick of Korn which has been applied to second order equations by E. Hopf, Schauder, and Morrey as well as the idea due to Nirenberg of injecting into Korn's trick the singular integral theorems of Calderon-Zygmund. (Received July 19, 1955.)

697t. F. H. Brownell: A Gaussian averaged error estimate for a plane lattice point problem.

It has long been known that the number $F(x)$ of lattice points $\left(m_{1}, m_{2}\right), m_{i}$ being integers, in the plane which satisfy $m_{1}^{2}+m_{2}^{2} \leqq x$ for $x>0$ is given by the equation $F(x)=\pi x+R(x)$. For the remainder $R(x)$ it is known as $x \rightarrow+\infty$ that $R(x)=O\left(x^{\beta}\right)$ for some $\beta<1 / 3$ (van der Corput), that $R(x)$ is not $o\left(x^{1 / 4}\right)$ (Hardy), and that $(1 / x)$ - $\int_{0}^{x}|R(t)| d t=O\left(x^{1 / 4}\right)$ (Cramér). As a consequence of an investigation of the extended asymptotic distribution of eigenvalues of the membrane equation, we have found that $R(x)=\widetilde{O}\left(x^{-r}\right)$ for every $r>0$. The precise definition of this Gaussian averaged error estimate denoted by $\widetilde{O}\left(x^{-r}\right)$ is that $\left|\int_{1 / 2}^{\infty} \exp \left(-\rho^{2}(\ln x / y)^{2}\right) d R(y)\right| \leqq M_{\rho} x^{-r}$ over $x \geqq 1 / 2$ for some finite $M_{\rho}$ existing for every $\rho>0$, and that $\psi_{R}(S)=\int_{1 / 2}^{\infty} Y^{-8} d R(y)$ possesses an analytic continuation in $s$ from the region $\Re[s]>2$ throughout $\Re[s] \geqq 0$ for which $\psi_{R}(0)=-R(1 / 2)$. (Received July 5,1955 .)

698. Lamberto Cesari: Retraction of mappings from finitely connected Jordan regions.

Notations: $J, J_{0} \subset E_{2}$ finitely connected closed Jordan regions; $S=(T, J): p=T(w)$, continuous mapping from $J$ into $E_{n}$ (surface); $L(S)$ Lebesgue area of $S$; $\Gamma$ the decomposition of $J$ into maximal continua of constancy $g$ for $(T, J) ; K \subset J$ any continuum; $\{\gamma\}_{K T J}$ the collection of the components $\gamma$ of $J-K$. Properties $\mathrm{P}^{\prime}, \mathrm{P}_{1}, \mathrm{P}_{2}$ of $K$ with respect to $(T, J):\left(\mathrm{P}^{\prime}\right) K=\sum g, g \in \Gamma ;\left(\mathrm{P}_{1}\right) k \subset g, g \in \Gamma$, for every component $k$ of $F(\gamma)=\bar{\gamma}-\gamma$ and $\gamma \in\{\gamma\}_{K T J} ;\left(\mathrm{P}_{2}\right) F(\gamma)$ is a continuum, for every $\gamma \in\{\gamma\}_{K T J}$. I. If $K C J$ has properties $\mathrm{P}^{\prime}, \mathrm{P}_{1}$ with respect to $(T, J)$, then there is some $J_{0}$ with $K \subset J_{0} \subset J$ such that $K$ has properties $\mathrm{P}^{\prime}, \mathrm{P}_{1}, \mathrm{P}_{2}$ with respect to $\left(T, J_{0}\right)$. Then a mapping $\left(T_{0}, J_{0}\right)$ is the retraction of $(T, J)$ with respect to $K$ in $J_{0}$ if $T_{0}=T$ on $K, T_{0}(w)$ $=T[F(\gamma)]$ for every $w \in \gamma$ and $\gamma \in\{\gamma\}_{K T J_{0}}$. Now $K \subset J$ is said to be a fine-cyclic element (set) (fce) for ( $T, J$ ) if (i) $K$ is a continuum; (ii) $\mathrm{P}^{\prime}$ and $\mathrm{P}_{1}$ hold, and $K \notin \Gamma$; (iii) $K$ is minimal with respect to (i), (ii). Then $\sigma=\left(T_{0}, J_{0}\right)$ [retraction of $(T, J)$ with respect to $K$ in a region $J_{0}$ as above] is said to be a fine-cyclic element (surface) (fce) of $S=(T, J)$. II. The collection of all (fce) of $(T, J)$ is countable; III. For every fce $K$ there is a cyclic element $K_{0}$ with $K \subset K_{0}\left[K=K_{0}\right.$ if $J$ is simply connected]. IV. $L(S)$ $=\sum L(\sigma)$ where $\sum$ ranges over all fce of $S$ (fine-cyclic additivity theorem). (Received July 15,1955 .)

699t. Paul Civin and Bertram Yood: Boundaries for families of continuous functions.

Let $H$ be a multiplicative semi-group of real or complex bounded continuous functions on a topological space $E$. If there exists a unique minimal closed subset $E_{1}$ of $E$ such that for all $f \in H$, sup $|f(t)|, t \in E_{1}$, is the same as $|f(t)|, t \in E$, then $E_{1}$ is called the Silov boundary of $E$ relative to $H$. A characterization and an analytic representation are obtained for the Silov boundary of $E$ relative to $H$, whenever the latter exists. (Received July 5, 1955.) 
700t. Paul Civin and Bertram Yood: Ideals in semi-groups of continuous functions.

Let $C$ be an abstract multiplicative semi-group which is known to be the semigroup of the continuous functions vanishing at infinity on some locally compact space $X$. Sub-semi-groups $B$ of $C$ are characterized in which suitable classes of multiplicative ideals are in 1-1 correspondence with the closed sets of $X$. The ideals used are similar to those used by Milgram (Duke Math. J. vol. 16 (1949) pp. 377-383) and Yood (to appear in the Duke Math. J.) in the compact case. Applications are made to regular Banach algebras. (Received July $5,1955$. )

\section{1t. Paul Civin and Bertram Yood: Invariant functionals.}

Let $E$ be a normal linear space and $G$ a solvable group of bounded linear operators on $E$. Conditions for the existence of a nontrivial bounded linear functional $x^{*}$ invariant under $G$ were given by Yood (Proc. Amer. Math. Soc. vol. 2 (1951) pp. 225-233). Related problems for $E$ a real linear space were treated by Klee (Pacific Journal of Mathematics vol. 4 (1954) pp. 37-46). In this paper $E$ is taken to be a Banach space and category arguments are used to improve the sufficient conditions for the existence of the functional $x^{*}$. The conditions involve hypotheses on the orbits of $x \in E$ under $G$ rather than the metric properties of $G$ as heretofore. (Received July $5,1955$. )

\section{2t. Harvey Cohn: Perturbation modular functions. II.}

The author continues the study of perturbation modular functions defined in an earlier abstract (Bull. Amer. Math. Soc. Abstract 61-2-231). The following application is made: Consider a Fuchsian group of transformations $S z=(a z+b) /(c z+d)$ with real unimodular transformation matrices $S$ and a fundamental domain intersecting the real axis at one or more cusps. Consider further the regular modular functions $f(z)$ of degree -4 , i.e., $f(S z)(c z+d)^{-4}=f(z)$. Obviously every sum $E(z)=\sum(c z+d)^{-4}$ is such an $f(z)$ (where the summation is taken over cusps $-d / c$ equivalent under $S$ to a fixed cusp). Conversely, however, not every $f(z)$ is a linear combination of these sums. The sums (or rather linear combination with real coefficients) can be characterized by the fact that their values possess a third primitive $P(z)$ (i.e., $P^{\prime \prime \prime}(z)$ $=E(z)$ ), such that $P(z)-(c z+d)^{2} P(S z)$ is a quadratic polynomial in $z$ with real coefficients. The proof of this result on the dimensionality of differentials is accomplished by a geometrical construction, i.e., it is shown that any $P(z)$ of this type can be arrived at only by the perturbation process defined in the earlier abstract. (Work supported by ONR contract.) (Received April 14, 1955.)

\section{V. F. Cowling: On Borel summability.}

Denote by $R\left(h_{1} \psi_{1}, \psi_{2}\right)$ a region in the complex $w$-plane which contains the origin as an interior point and whose boundary consists of two rays $I_{1}=I\left(h, \psi_{1}\right)$ and $I_{2}=I\left(h, \psi_{2}\right)$ emanating from the point $h,-1<h<0$, making angles $\psi_{1}$ and $\psi_{2}$ respectively with the positive direction of the axis of reals. The following theorem is proved. Let $a(w), w=h+R e^{i \psi}$, be analytic in $R\left(h, \psi_{1}, \psi_{2}\right)$ and there satisfy the condition $|a(w) / \Gamma(w+1)|=O\left(|w| K e^{\delta|w|}\right)$ for some $\delta(0<\delta<\pi)$ and some real $k, \psi_{1}$ and $\psi_{2}$ satisfying the inequalities $\pi / 2<\psi_{1}<\pi,-\pi<\psi_{2}<-\pi / 2$, $\sin \psi_{1}>2 \delta \pi /\left(\pi^{2}+\delta^{2}\right)$, and $\sin \psi_{2}<-2 \delta \pi /\left(\pi^{2}+\delta^{2}\right)$. Suppose also that $\operatorname{Lim} \operatorname{Sup}_{m}|a(n) / \Gamma(n+1)|^{1 / n}=1$. Then $f(z)=\sum a(n) z^{n}$ is Borel integral summable for each $z$ for which $\delta$ Csc $\psi_{1}<\operatorname{Arg} z$ $<2 \pi-\delta\left|\operatorname{Csc} \psi_{2}\right|$ and the Borel integral defines a function analytic for each $z$ for which $\delta \operatorname{Csc} \psi_{1}-\pi / 2<\operatorname{Arg} z<5 \pi / 2-\delta\left|\operatorname{Csc} \psi_{2}\right|$. This theorem is a generalization of a 
particular case of a theorem due to S. Bernstein (Bull. Sci. Math. vol. 63 (1928)). A similar theorem for Dirichlet is obtained. (Received July 13, 1955.)

704. Philip Davis and H. O. Pollak (p): On the analytic continuation of mapping functions.

The problem of this paper is to study the relationships between the continuability of various mapping functions of a domain $B$ with analytic boundary and the moments $\iint_{B} z^{n} \overline{g(z)} d A$, where $g(z)$ is an arbitrary function analytic in $B$. Let $\bar{t}(z)$ denote $\overline{t(\bar{z})}$ for any analytic function $t(z)$, and let $h(z)=\int_{0}^{z} g(u) d u$. Then a typical theorem reads as follows: Let $B$ be a simply connected domain with analytic boundary and containing the origin, and let $m(z)$ map the unit circle onto $B$ with $m(0)=0$. Then $\bar{h}(\bar{m}(z))$ is entire if and only if $\lim _{n \rightarrow \infty}\left|\iint_{B} z^{n} \overline{g(z)} d A\right|^{1 / n}=0$. In case the lim sup of the preceding expression does not vanish, it may be identified by introducing a certain sequence of "Radienbilder" of $B$ and finding the largest to which $\bar{h}(\bar{m}(z))$ continues analytically. (Received July 13, 1955.)

\section{Ruth M. Davis: On The Euler-Poisson-Darboux equation.}

Let $u(x)$ denote $u\left(x_{0}, x_{1}, \cdots, x_{n-1}\right)$ and let $k$ be a real parameter, $-\infty<k<\infty$. The explicit solution of a regular Cauchy problem for the $n$-dimensional EulerPoisson-Darboux equation (E.P.D. equation) written as (1) $L[u]=u_{x_{0} x_{0}}+k x_{0}^{-1} u_{x_{0}}$ $-\sum_{i=1}^{n-1} u_{x_{i} x_{i}}=0$, (2) $u=f\left(x_{1}, x_{2}, \cdots, x_{n-1}\right), u_{x_{0}}=0$ for $x_{0}=t_{0}>0$, is found by an extension of the method of Marcel Riesz. The essential point of this extended method is to find a function $V^{\alpha}(x ; y)$ depending on a parameter $\alpha$ for which $M\left[V^{\alpha+2}\right]=V^{\alpha}$ and such that $V^{\alpha}$ and its first derivatives vanish on the surface of the characteristic cone with vertex $x$. Here $M$ is the adjoint operator to $L$. The method of solution which relies on analytic continuation of divergent improper integrals leads to final formulas of entirely different character for odd and even dimensions. It is seen that there is no Huyghen's principle for $n$ odd but for $n$ even Huyghen's principle holds when $k$ is even and $0 \leqq k \leqq n-2$. For certain values of $k$ and $n$ the solution of the singular Cauchy problem for (1) is obtained immediately by simple limiting processes. Finally it should be noted that once the function $V^{\alpha}$ is found the solution to any regular correctly set Cauchy problem for either the homogeneous or the inhomogeneous E.P.D. equation should be readily obtainable. (Received June $13,1955$. )

706. M. M. Day: Every L-space is isomorphic to a strictly convex space.

A normed space $B$ is called $\mathrm{sc}(\mathrm{sm})$ if it is isomorphic to a space with strictly convex (smooth) unit sphere. A recent paper (Day, Trans. Amer. Math. Soc. vol. 78 (1955) pp. 516-528) showed that $L(\mu)$, the space of $\mu$-summable functions on a measure space $X$, is sm if and only if $\mu(X)$ is $\sigma$-finite. In this note other results from that paper are used with a theorem of Maharam on the structure of measure algebras to prove that every $L(\mu)$ is sc. (Received June 15,1955 .)

707. Allen Devinatz (p) and A. E. Nussbaum: On the permutability of normal operators.

It is proved that if $N_{1}, N_{2}$, and $N$ are (in general unbounded) normal operators acting in a Hilbert space such that $N_{1} N_{2}=N_{2} N_{1}=N$, then $N_{1}$ and $N_{2}$ commute; i.e. the canonical resolutions of the identity of $N_{1}$ and $N_{2}$ commute. The method of proof is analogous to the one used by the authors in collaboration with J. von Neumann in 
a similar theorem for self-adjoint operators (cf. On the permutability of self-adjoint operators by A. Devinatz, A. E. Nussbaum and J. von Neumann, to appear in Ann. of Math.). Further, it is shown how this theorem yields a spectral representation of an $n$-parameter semi-group of normal operators: Let $\left\{N_{x}\right\}$ be a weakly continuous semigroup of normal operators (in general unbounded), where the index $x=\left(x_{1}, x_{2}, \cdots, x_{n}\right)$ ranges over the semigroup which is the Cartesian product $I_{m}^{+} \times E_{n-m}^{+}$where $I_{m}^{+}$is the set of vectors in Euclidean $m$-space with non-negative integer components and $E_{n}^{+}$ is the set of vectors in Euclidean $(n-m)$-space with non-negative components such that $x \neq(0,0, \cdots, 0)$. Then there exists a unique $2 n$-parameter resolution of the identity $\{K(t, s)\}=\left\{K\left(t_{1}, t_{2}, \cdots, s_{1}, s_{2}, \cdots, s_{n}\right)\right\}$ such that $K(t, s)=0$ if any $t_{k}<0, k=1,2, \cdots, n$, and $N_{x}=\int t^{x} e^{i x \cdot s} d K(t, s)$, where $t^{x}=\prod_{1}^{n} t_{k}^{x}, x \cdot s=\sum_{1}^{n} x_{k} s_{k}$. (Received June 24, 1955.)

708. L. E. Dubins: A Radon-Nikodym type theorem for vector valued measures.

Let $M$ be the measure ring of a probability space and let $S$ be the dual of a metrizable topological vector space $B$. A notion of generalized random variable is defined. The author determines necessary and sufficient conditions for a function defined on $M$ with values in $S$ to be the indefinite integral of a suitably measurable, integrable generalized random variable. (Received July 19, 1955.)

\section{9t. R. J. Duffin: Infinite programs.}

Most of the development work on linear programming theory has been confined to finite programs. A program is termed finite if it involves only a finite number of variables and a finite number of constraint inequalities on these variables; otherwise, a program is termed infinite. In this paper the interesting duality theory which has been developed for finite linear programs is extended in such a manner that it applies to infinite linear programs. The theory, so extended, finds application to integral inequalities of the following type. Let $A(s, t)$ and $a(s)$ be continuous functions and let $A(s, t)=-A(t, s)$. The problem is to minimize $f=\int_{0}^{1} a(t) x(t) d t$ for positive functions $x(t)$ such that $\int_{0}^{1} A(s, t) x(t) d t+a(s)>0$ for $s$ in the interval $(0,1)$. It is shown that the greatest lower bound of $f$ is zero. (Received July 14, 1955.)

\section{Albert Edrei: Analogue of a theorem of Boas and Pólya.}

Let $f(z)$ be holomorphicfor $|z|<1$ and $f(z) f^{\prime}(z) f^{\prime \prime}(z) \neq 0$. Assume $\mid\left(f(0) f^{\prime \prime}(0) /\left\{f^{\prime}(0)\right\}^{2}\right)$ $-1 \mid \geqq \kappa\left(0<\kappa \leqq e^{-1}\right)$. Then $\left|f\left(z_{1}\right) / f\left(z_{2}\right)\right| \leqq \exp \left(A \kappa^{-32 / 3}\left|z_{1}-z_{2}\right|\right)$ where $A$ is an absolute constant and $z_{1}, z_{2}$ any two points of the disk $|z| \leqq 1 / 4$. The index $m$ is said to be critical if $f^{(m)}(z) f^{(m+1)}(z) f^{(m+2)}(z) \neq 0$ in $|z|<1$, and if $\mid\left(f^{(m)}(0) f^{(m+2)}(0) /\left\{f^{(m+1)}(0)\right\}^{2}\right)$ $-1 \mid \geqq \kappa$. The above result and definition lead to the following analogue of a theorem of Boas and Pólya [Duke Math. J. vol. 9 (1942) p. 407]. Let $f(z)$ be holomorphic in the unit disk and let $\left\{n_{k}\right\}$ be an infinite sequence of critical indices (corresponding to a fixed value of $\kappa(>0))$. Then (i) if $n_{k}-n_{k-1}=O(1), f(z)$ is an entire function of order one and finite type; (ii) if $n_{k}-n_{k-1}=O\left(n_{k}^{\delta}\right)$ where $\delta$ is fixed, $0<\delta<1$, then $f(z)$ is an entire function of finite order not exceeding $1 /(1-\delta)$; (iii) if $n_{k}-n_{k-1}=O\left(n_{k}\right)$, then $f(z)$ is an entire function. (Received July $18,1955$.

711. H. W. Ellis: On the basis problem for function spaces determined by length functions.

In a paper entitled Haar functions and the basis problem for Banach spaces*, to appear in the Journal of the London Mathematical Society, Israel Halperin and the 
author defined generalized Haar functions on a general (not necessarily metric) measure space $S$ and showed that there is a countable basis of generalized Haar functions in separable $L^{\lambda}$ spaces of real valued functions on $S$ for suitable $\lambda$, including $\lambda$ a levelling length function. (For the definition of length functions and $L^{\lambda}$ spaces see H. W. Ellis and Israel Halperin, Canadian Journal of Mathematics vol. 5 (1953) pp. 576-592.) The existence of a countable basis in vector valued Banach spaces $L^{\lambda^{\prime}}\left(L^{\lambda}\right)$ is considered. It is shown that if $L^{\lambda^{\prime}}$ and $L^{\lambda}$ are spaces of real valued functions on the general measure spaces $S$ and $S^{\prime}$ and if $L^{\lambda^{\prime}}\left(L^{\lambda}\right)$ is separable then $L^{\lambda^{\prime}}\left(L^{\lambda}\right)$ is equivalent to a Banach space $L^{\lambda^{\prime} \lambda}$ of real valued functions on the Cartesian product space $S \times S^{\prime}$. The existence of a countable basis of generalized Haar functions in $L^{\lambda^{\prime} \lambda}$ when both $L^{\lambda}$ and $L^{\lambda^{\prime}}$ satisfy the conditions of ${ }^{*}$ is then shown. (Received July 6,1955 .)

\section{N. J. Fine: Cesàro summability of Walsh-Fourier series.}

The Walsh-Fourier series of an integrable function $f(x)$ is $(C, 1)$ summable to $f(x)$ almost everywhere. For $f \in L^{p}, p>1$, a proof was indicated by Paley [Proc. London Math. Soc. (2) vol. 34 (1932) pp. 241-279]. (Received May 16, 1955.)

\section{Tomlinson Fort: Limits of the characteristic values for certain boundary-value problems associated with difference equations.}

This paper is concerned with the $n$-dimensional generalization of $\Delta\{k(i-1)$ - $\Delta y(i-1)\}+\left[\lambda / m^{2}-s(i)\right] y(i)=0$ subject to boundary conditions; $y(1)=c_{1} y(0)$, $y(m+1)=c_{2} y(m)$. Several theorems are proved with reference to the characteristic equation. Then if $k(i)=\bar{k}\left(x_{i}\right)$ where $x_{i+1}-x_{i}=1 / m$ and $\operatorname{limit}_{m \rightarrow \infty} \bar{k}\left(x_{i}\right)=K(x)$ and if the characteristic equation is transformed so that its roots are multiplied by $1 / m$ and if $m^{2} / \lambda$ is denoted by $\rho$ then explicit forms for the limits of the coefficients of the equation in $\rho$ are obtained. Several theorems relative to the characteristic values for the difference problem and the approximated differential problem are then proved. The $n$-dimensional case involves only expected variations from the one-dimensional case. (Received July 18, 1955.)

\section{4t. Evelyn Frank: $A$ new class of continued fraction expansions} for the ratios of hypergeometric functions.

A new class of continued fraction expansions for the ratios of two Gauss hypergeometric functions is described in detail. A number of such ratios are expanded, and the convergence of the corresponding continued fractions is determined as well as the functions to which the continued fractions converge in various regions. Many special relations are thus obtained between functions, finite continued fractions, and infinite continued fractions. Specialized continued fraction expansions and specialized functions are discussed, and integral representations for such continued fractions are found. (Received July 6, 1955.)

\section{5t. Casper Goffman: Convergence in area of integral means.}

The theorem of Rad6 that the Lebesgue areas of the surfaces defined by the integral means of a continuous $f(x, y)$ converge to the Lebesgue area of the surface given by $f(x, y)$ is extended to summable functions. This allows a definition of area of surfaces associated with summable functions to be made which agrees with a definition given previously by L. Cesari (Annali Scuola Normale Superiore Pisa (2) vol. 5 (1936) pp. 299-313) and the author (Rend. Circ. Mat. Palermo (2) vol. 2 (1953) pp. 203-235). (Received June 6, 1955.) 


\section{Michael Golomb: Spectral properties of commutators.}

Let $A, B$ be linear operators on a Banach space $\mathfrak{X}, C=A B-B A$ their cummutator, and assume $A C=C A$. The conjecture that the spectrum $\sigma(C)$ consists of 0 alone is confirmed in this paper by the following results. (1) $C=0$ if $\mathfrak{X}$ is Hilbert and one of the operators $A, B, C$ is normal. (2) $C=0$ if $\mathfrak{X}$ is Hilbert and $A$ or $B$ is maximal hermitian (in this case there are certain additional hypotheses on the domains of the nonbounded $A, B, C$ ). (3) $C=0$ if $C=\sum_{i} \lambda_{i} E_{i}$ where $E_{i} E_{j}=\delta_{i j} E_{i}$. (4) $\sigma(C)$ has no component not connected with 0 . (5) If $C$ is a "spectral operator of finite type" defined to possess a certain spectral decomposition then $\sigma(C)=[0]$. To prove that certain operators, not normal nor completely continuous, are spectral operators of finite type on the Hilbert space $\mathfrak{X}$ the following theorem is proved. If $N=\int_{\sigma} z d E_{z}$ is normal, $\sigma=\sigma_{1} \dot{+} \sigma_{2} \dot{\dagger} \cdots$ where the $\sigma_{i}$ are both open and closed in $\sigma$, and $R$ is such that $R \int_{\sigma_{i}} d E_{z}=\int_{\sigma_{i}} d E_{z} R$ is nilpotent for every $i$ then $A(N+R)=(N+R) A$ implies $A E_{z}=E_{z} A$ for every $z$. (Received July 18,1955 .)

717. P. E. Guenther: Canonical solutions of a $q$-difference system. Preliminary report.

It is known that the $q$-difference system $Y(q x)=A(x) Y(x)$ where the elements of the coefficient matrix $A(x)$ are functions analytic except possibly for poles at $x=\infty$ may be reduced to canonical form $Y(q x)=C(x) Y(x)$ where the coefficient matrix $C(x)$ involves only polynomial or zero elements (Proc. Nat. Acad. Sci. U.S.A. vol. 27 (1941) pp. 218-222). Starting with this latter form of the $q$-difference system analytic solutions are determined by a variety of methods employed by Birkhoff, Carmichael, Nörlund, and others in the case of additive difference equations. The relations between these solutions are studied and their asymptotic representation by the complement of formal series solutions is established. In particular, the convergent part of the formal series complement is found to be predictable from the array of vanishing elements in $C(x)$. (Received July 15, 1955.)

\section{Meyer Jerison: Martingale formulation of ergodic theorems.}

Let $f$ be an integrable function on a $\sigma$-finite measure space $X, T$ a 1-1 measurepreserving transformation of $X$, and $N$ the set of non-negative integers with measure 1 assigned to each integer. On the product space $X \times N$ with the product measure, a sequence of functions integrable on sets of finite measure is defined: $h_{k}(x, 0)=k^{-1}$ - $\sum_{i=0}^{k-1} f\left(T^{i} x\right), h_{k}(x, n)=h_{k}\left(T^{-n} x, 0\right)$ if $n<k$, and $h_{k}(x, n)=f(x)$ if $n \geqq k$. The ergodic theorem asserts the existence of $\lim _{k \rightarrow \infty} h_{k}(x, n)$ almost everywhere on $X \times N$. The sequence $\left\{h_{k}\right\}$ is a martingale [Doob, Stochastic processes, New York, 1953] with the index going backward, but the existence of $\lim _{k \rightarrow \infty} h_{k}(x, n)$ cannot be inferred from the standard martingale convergence theorem because $X \times N$ has infinite measure. A similar formulation of the generalized ergodic theorem [Hurewicz, Ann. of Math. vol. 45 (1944) pp. 192-206] is given by replacing the product measure on $X \times N$ with one that reflects the effect of a transformation which does not preserve measure. This formulation sheds light upon the relation between the ergodic theorems and the martingale convergence theorem. (Received July 14, 1955.)

719. R. V. Kadison: Operator algebras with a faithful weakly-closed representation.

A $W^{*}$-algebra is defined to be a $C^{*}$-algebra in which each bounded, monotoneincreasing, directed sequence $\left\{A_{\gamma}\right\}$ of operators has a least upper bound $A$ and which 
has a family of states $\omega$ such that $\left\{\omega\left(A_{\gamma}\right)\right\}$ has the directed limit $\omega(A)$ and such that if $\omega(B)=0$ for some positive operator $B$ in the algebra and each state of the family then $B=0$. Main theorem: A $C^{*}$-algebra has a faithful representation as a ring of operators if and only if it is a $W^{*}$-algebra. (Received June 6,1955 .)

720. M. S. Klamkin: On a graphical solution of the general second order linear differential equation.

The general second order linear differential equation can be transformed into the canonical form $\left[D^{2}+\phi(x)\right] y=F(x)$. This $2 \mathrm{~d}$ order equation is equivalent to the following set of 1st order equations: $D n=n^{2}+\phi(x),[D+n] y=r$, and $[D-n] r=f(x)$; which are then solved graphically. (Received July 18, 1955.)

721t. V. L. Klee, Jr.: Solution of a problem of E. M. Wright on convex functions.

With $R$ denoting the real number field and $f$ a function on $R$ to $R$, consider the following two statements about $f$ : (A) $f(x+d)-f(x) \geqq f(y+d)-f(y)$ for all $d>0$ and $x>y$. (B) $f(x / 2+y / 2) \leqq f(x) / 2+f(y) / 2$ for all $x, y$. It is well known that (A) implies (B), and that for continuous $f$ the two are equivalent. In a recent paper [An inequality for convex functions, Amer. Math. Monthly vol. 61 (1954) pp. 620-622], Wright asked whether there is a function $f$ for which (B) is true and (A) is false. It is proved here that if $\tau$ is an arbitrary additive, rationally homogeneous isomorphism of $R$ onto $R^{2}$, and if $f(x)=|\tau(x)|^{2}$ for each $x \in R$, then $f$ is such a function. (This note will appear in the American Mathematical Monthly.) (Received June 15, 1955.)

722. R. E. Langer: On the construction of related differential equations.

Linear differential equations that depend upon a parameter $\lambda$ are commonly of the form (1) $d^{n} u / d z^{n}+\lambda p_{1}(z, \lambda) d^{n-1} u / d z^{n-1}+\cdots+\lambda^{n} p_{n}(z, \lambda) u=0$, with each coefficient $p,(z, \lambda)$ a polynomial or power series in $1 / \lambda$. For many purposes the forms of the solutions of such an equation, when $|\lambda|$ is large, must be determined. That can be done by classical methods, if the $z$-region in which the equation is being considered is one in which the roots of the auxiliary algebraic equation (2) $\chi^{n}+p_{1}(z, \infty) \chi^{n-1}+\cdots$ $+p_{n}(z, \infty)=0$ maintain an invariant configuration, i.e. roots that are single at any $z$ are so for all $z$, ones that are multiple, are identically so in $z$. Other methods are required if the z-region contains a turning point (transition point) at which the auxiliary roots have a different configuration than elsewhere. A method that is then applicable is based upon a so-called "related equation." This is a differential equation that fulfills the specifications (a), its coefficients are identical with those of the given equation to the terms in some specified power in $1 / \lambda$, and (b), its solution forms are known. This paper considers the differential equation (1) in a $z$-region in which $(n-m)$ roots of the equation (2) remain simple, whereas the other $m$ roots, $\chi_{1}, \chi_{2}, \cdots, \chi_{m}$, are involved in multiplicities at a turning point. It shows how a related equation is to be constructed if such an equation is known, or constructible, for the differential equation of the lower order $m$ whose auxiliary equation has the roots $\chi_{1}, \chi_{2}, \cdots, \chi_{m}$. (Received July 11, 1955.)

723t. J. J. Levin: On singular perturbations related to conditional stability.

The behavior, as $\epsilon \rightarrow 0+$, of the solutions of the system of real differential equa- 
tions (1) $d x / d t=f(x, y, t, \epsilon), \epsilon d y / d t=A y+g(x, y, t, \epsilon)$ is investigated. It is assumed that $x$ and $y$ are vectors of $m$ and $n$ components respectively, that $A$ is a constant $n$ by $n$ matrix $k$ of whose characteristic roots have negative real parts, while the remaining $n-k$ characteristic roots have positive real parts, and that $f$ and $g$ satisfy several mild smoothness hypotheses. It is shown that there exists a $k$-dimensional initial manifold in $y$ space, which depends on $\epsilon$ and the initial $x$ vector, such that if the initial $y$ vector lies on the manifold then the solution of (1) exists, for all sufficiently small $\epsilon>0$, over a fixed $t$ interval which does not depend on $\epsilon$. Further properties of this initial manifold and of solutions starting near it are established. (Received July 13, 1955.)

\section{A. J. Lohwater and George Piranian ( $\mathrm{p})$ : On a conjecture of} Lusin.

A point $e^{i \theta}$ is called an $L$-point of a function $f(z)$, regular in $|z|<1$, if for every $t$ in $0<t<1$ the (possibly many-sheeted) image of the disc $\left|z-t e^{i \theta}\right|<1-t$ under the mapping $w=f(z)$ has infinite area. It was conjectured by N. Lusin [Doklady Akad. Nauk $56(1947)$ ] that there exists a bounded analytic function $f(z)$ for which the set of $L$-points on $|z|=1$ is of measure $2 \pi$. It is shown, by means of a Taylor series, that there exists a function $f(z)$, regular in $|z|<1$ and continuous in $|z| \leqq 1$, for which every point of $|z|=1$ is an $L$-point. It is also shown that there exists a function $f(z)$, regular in $|z|<1$ and continuous in $|z| \leqq 1$, which maps $|z|<1$ into a region of infinite area, but which has no $L$-points on $|z|=1$. (Received May 23, 1955.)

\section{R. M. McLeod: On the zeros of the derivatives of some entire} functions.

Let $\mathcal{L}(f)$ be the set of limit points of the zeros of the derivatives of the entire function $f$. Let $\mathcal{F}_{1}$ be the class of entire functions $f(z)=z^{m} P(z) \exp (Q(z))$ where $Q(z)$ is a polynomial of degree $q$ and $P(z)$ is a canonical product of genus $p$ with $0 \leqq p \leqq q-2$. P6lya (Math. Zeit. vol. $12(1922)$ pp. 36-60) found $\mathcal{L}(f)$ for those $f \in \mathcal{F}_{1}$ for which $P(z)$ is a polynomial. Let $Q(z)=\sum_{k=0}^{a} b_{k} z^{k}$ with $b_{0}>0$. Set $w=|w| e^{i \theta}=z+b_{1} /\left(q b_{0}\right)$ and $S_{k}(t)=\{z|| w|>0,| \theta-2 \pi k / q \mid<t\}$. Let $R$ be the complement of $\bigcup_{k=0}^{\alpha-1} S_{k}(\pi / q)$. If $f \in \mathcal{F}_{1}$ and if $f$ has at most a finite set of zeros in $\bigcup_{k=0}^{a-1} S_{k}(t)$ for some positive $t$, then by methods similar to those of Polya it is proved that $\mathcal{L}(f)=R$. Similar results are obtained for the class $\mathcal{F}_{2}$ of functions $f(z)=z^{m} P(z) \exp \left(b_{0} z^{2}+b_{1} z+b_{2}\right), b_{0}>0$, where $P(z)$ is a canonical product of genus one with zeros $a_{k}$ satisfying $\sum_{k=1}^{\infty}\left|\operatorname{Re}\left(1 / a_{k}\right)\right|<\infty$. Moreover, it is shown that there are functions $f \in \mathcal{F}_{1}$ for which $R$ is a proper subset of $\mathcal{L}(f)$. (Received July 15, 1955.)

\section{6t. G. W. Mackey: Unitary representations of group extensions.}

In an earlier paper (Proc. Nat. Acad. Sci. U.S.A. (1949) pp. 537-545) the author used the theory of induced representations to study (unitary) representations of semi-direct products of separable locally compact groups in the special case in which the normal subgroup is required to be abelian. In the present paper this work is extended to a study of the connection between the representations of $K, G$, and $G / K$ where $K$ is a (not necessarily abelian) closed normal subgroup of $G$ and $G$ is an arbitrary extension of $K$ by $G / K$. The representations of $G$ may depend upon the projective representations of $G / K$ and its subgroups. However the whole theory may be developed $a b$ initio for projective representations so that the results may be used inductively to study complicated groups. In particular a theory of induced projective representations is presented which extends previous work of the author on induced 
representations. $K$ is not allowed to be completely arbitrary but must have a suitably regular deconıposition theory for its representations. Investigation of this point leads to a refinement of existing work on decomposition theory and to a division of groups into those with "smooth" and "rough" duals according to the behavior of a certain natural Borel structure in the set of equivalence classes of irreducible representations. (Received July $18,1955$. )

\section{7t. G. R. MacLane: On the zeros of the derivatives of an entire function.}

Pólya (Bull. Amer. Math. Soc. vol. 49 (1943) p. 179) has defined the final set $L_{f}$ of an entire function $f(z)$ as follows: $z_{0} \in L_{f}$ if and only if for each neighborhood $U$ of $z_{0}$ there are infinitely many of the functions $\left\{f^{(n)}(z)\right\}$ which have zeros in $U$. It is a simple consequence of known facts that either $f(z)=a \exp (b z)$, in which case $L_{f}$ is void, or $\infty \in L_{f}$. The present paper proves the following theorem. Let $K$ be any compact subset of the sphere such that $\infty \in K$. Then there exists an entire function $f$ such that $L_{f}=K$. Furthermore, $f$ may be chosen to satisfy any one of the following conditions: (1) if $p(r), r>0$, is such that $\lim _{r \rightarrow \infty} r^{-n} p(r)=\infty, n>0$, then $|f(z)|<p(|z|)$; (2) $f$ is of order $\mu, 0<\mu<\infty$; (3) $f$ is of arbitrarily rapid growth. (Received July 11 , 1955.)

728t. J. S. MacNerney: Hellinger integrals in inner product spaces. I.

An inner product space is an ordered pair $\{S, Q\}$ such that $S$ is a (complex) linear space and $Q$ is an inner product for $S ; B\{S, Q\}$ is the space of continuous linear transformations in $\{S, Q\}$, normed in the usual way. It is assumed throughout this sequence that $\left\{S_{0}, Q_{0}\right\}$ is a complete inner product space. The idea of a certain Hellinger integral is extended to obtain an integral denoted by $\int_{0}^{1} Q_{0}\left(d f,[d \phi]^{-1} d g\right)$, where each of $f$ and $g$ is a function from $[0,1]$ to $\left\{S_{0}, Q_{0}\right\}$ and the function $\phi$, from $[0,1]$ to $B\left\{S_{0}, Q_{0}\right\}$, is nondecreasing in the sense that $\phi(b)-\phi(a)$ is Hermitian and non-negative for each subinterval $[a, b]$ of $[0,1]$. LEMMA: For $H$ and $K$ nonnegative Hermitian in $B\left\{S_{0}, Q_{0}\right\}, Q_{0}\left(x, H^{-1} x\right)+Q_{0}\left(y, K^{-1} y\right) \geqq Q_{0}\left([x+y],[H+K]^{-1}\right.$ - $[x+y])$ where $H^{-1} x$ is any point $u$ in $S_{0}$ such that $H u=x$, etc. Let $H$ be non-negative Hermitian in $B\left\{S_{0}, Q_{0}\right\}$ and $S$ be the linear space of all functions $f$ from $[0,1]$ to $S_{0}$ such that $f(0)$ is in $H\left(S_{0}\right)$ and $\int_{0}^{1} Q_{0}\left(d f,[d \phi]^{-1} d f\right)$ exists. Let $Q(f, g)=Q_{0}\left(f[0], H^{-1} g[0]\right)$ $+\int_{0}^{1} Q_{0}\left(d f,[d \phi]^{-1} d g\right)$ for $f$ and $g$ in $S$. The inner product space $\{S, Q\}$ is complete only in case $H\left(S_{0}\right)$ and all $[\phi(b)-\phi(a)]\left(S_{0}\right)$ are closed linear manifolds in $\left\{S_{0}, Q_{0}\right\}$. Let $K(s, t)=K(t, s)=H+\phi(s)$ for $0 \leqq s \leqq t \leqq 1$. For "fixed" $t$ in $[0,1]$ and $x$ in $S_{0}$, $K(\cdot, t) x$ is in $S$ and $Q(f[\cdot], K[\cdot, t] x)=Q_{0}(f[t], x)$ for $f$ in $S$. (Received July 29, 1955.)

729t. J. S. MacNerney: Hellinger integrals in inner product spaces. II.

Let $E$ be a set. A kernel in the inner product space $\{S, Q\}$ of functions from $E$ to $\left\{S_{0}, Q_{0}\right\}$ is a function $K$ from $E \times E$ such that each $K(s, t)$ is a linear transformation from $S_{0}$ to $S_{0}$ and, for each $t, x$ in $E \times S_{0}$, the function $K(\cdot, t) x$ belongs to $S$ and $Q(f[\cdot], K[\cdot, t] x)=Q_{0}(f[t], x)$ for all $f$ in $S$. There is a kernel in $\{S, Q\}$ only in case convergence in $\{S, Q\}$ (assumed complete) implies point-wise convergence over $E$ : $K$ is unique. A kernel $K$ must satisfy $\sum_{i, j} Q_{0}\left(x_{i}, K\left[t_{i}, t_{j}\right] x_{j}\right) \geqq 0$ for all finite sets $\left\{t_{i}\right\}$ in $E$ and $\left\{x_{i}\right\}$ in $S_{0}$, whence the values of $K$ are in $B\left\{S_{0}, Q_{0}\right\}$. If $K$ is a function from $E \times E$ to $B\left\{S_{0}, Q_{0}\right\}$ satisfying this condition, there is only one complete inner product 
space $\{S, Q\}$ of functions from $E$ to $\left\{S_{0}, Q_{0}\right\}$ in which $K$ is the kernel, and the set of $f$ of the form $f(\cdot)=\sum K\left(\cdot, t_{j}\right) x_{j}$ is dense in $\{S, Q\}$; a function $f$ from $E$ to $\left\{S_{0}, Q_{0}\right\}$ belongs to $S$ only if there is a number $N$ such that $\left|\sum Q_{0}\left(f\left[t_{i}\right], x_{i}\right)\right|^{2} \leqq N \sum Q_{0}\left(x_{i}\right.$, $\left.K\left[t_{i}, t_{j}\right] x_{j}\right)$ for finite sets $\left\{t_{i}\right\}$ and $\left\{x_{i}\right\}$ as above, in which case $Q(f, f)$ is the least such $N$; if $\left\{S_{0}, Q_{0}\right\}$ is separable and $E$ is a separable topological space and the functions in $S$ are weakly continuous, then $\{S, Q\}$ is separable. This extends earlier results [Bull. Amer. Math. Soc. Abstract 61-2-266] of the author. (Received July 29, 1955.)

730t. J. S. MacNerney: Hellinger integrals in inner product spaces. III.

A kernel system is a sequence $\{K, E, S, Q\}$ such that $K$ is the kernel in the complete inner product space $\{S, Q\}$ of functions from the set $E$ to $\left\{S_{0}, Q_{0}\right\}$. Let each of $\left\{K_{1}, E_{1}, S_{1}, Q_{1}\right\}$ and $\left\{K_{2}, E_{2}, S_{2}, Q_{2}\right\}$ be a kernel system. If $E_{1}=E_{2}$ then $S_{1}$ is a subset of $S_{2}$ only in case there is a positive number $N$ such that $N K_{2}-K_{1}$ is the kernel in some space. Suppose $E_{1}=E_{2}$ and $S_{1}$ is a subset of $S_{2}$ : the equation $Q_{1}(f, T g)=Q_{2}(f, g)$ for $f, g$ in $S_{1} \times S_{2}$ defines a continuous linear transformation $T$ from $\left\{S_{2}, Q_{2}\right\}$ to $\left\{S_{1}, Q_{1}\right\}$; $S_{1}=T\left(S_{2}\right)$ only in case either $S_{1}$ or $T\left(S_{2}\right)$ is a closed linear manifold in $\left\{S_{2}, Q_{2}\right\}$. Suppose $E_{1}$ is a proper subset of $E_{2}$ and $K_{1}$ is the contraction of $K_{2}$ to $E_{1} \times E_{1}: S_{1}$ is the set of contractions to $E_{1}$ of functions in $S_{2} ;\left\{S_{1}, Q_{1}\right\}$ is isomorphic to $\left\{S_{3}, Q_{3}\right\}$, where $S_{3}$ is the orthogonal complement in $\left\{S_{2}, Q_{2}\right\}$ of the set of $f$ in $S_{2}$ such that $f(t)=0$ for $t$ in $E_{1}$, and $Q_{3}$ is the contraction of $Q_{2}$ to $S_{3} \times S_{3}$. Suppose $S$ is a linear subspace of $S_{1}$ : there exists a kernel system $\{K, E, S, Q\}$ only in case there exist a positive number $N$ and an inner product $Q^{\prime}$ for $S$ such that $\left\{S, Q^{\prime}\right\}$ is complete and $Q_{1}(f, f) \leqq N Q^{\prime}(f, f)$ for all $f$ in $S$. For details in the one-dimensional case (where $B\left\{S_{0}, Q_{0}\right\}=\left\{S_{0}, Q_{0}\right\}$ ), see Aronszajn [Trans. Amer. Math. Soc. vol. 68 (1950) pp. 337-404]. (Received July 29, 1955.)

731t. J. S. MacNerney: Hellinger integrals in inner product spaces. IV.

The kernel system $\{K, E, S, Q\}$ is ideal provided, for each finite sequence $t=\left\{t_{i}\right\}_{1}^{n}$ in $E$, the set $M_{t}$ of all $f$ of the form $f(\cdot)=\sum_{1}^{n} K\left(\cdot, t_{j}\right) x_{j}$ is a closed linear manifold in $\{S, Q\}$. If $S_{0}^{n}$ is the $n$-fold direct product of $S_{0}$ with itself and if $Q_{0}^{n}(x, y)$ $=\sum_{1}^{n} Q_{0}\left(x_{i}, y_{i}\right)$, each of the following is a necessary and sufficient condition that a set $M_{t}$ (as above) be closed in $\{S, Q\}:(1)$ the transformation $H, H x=\left\{\sum K\left(t_{i}, t_{j}\right) x_{j}\right\}$ for $x=\left\{x_{i}\right\}$, maps $S_{0}^{n}$ onto a closed linear manifold in $\left\{S_{0}^{n}, Q_{0}^{n}\right\} ;(2)$ the set $V_{t}$, to which $x$ belongs only in case there is an $f$ in $S$ such that $x=\left\{f\left(t_{i}\right)\right\}$, is a closed linear manifold in $\left\{S_{0}^{n}, Q_{0}^{n}\right\} ;(3)$ for each $f$ in $S$ there is an $\left\{x_{i}\right\}$ in $S_{0}^{n}$ such that $\left\{f\left(t_{i}\right)\right\}=\left\{\sum K\left(t_{i}, t_{j}\right) x_{j}\right\}$ in which case $\sum K\left(\cdot, t_{j}\right) x_{j}$ is the projection $P_{t} f$ of $f$ on $M_{t}$. In the latter notation, if $\{K, E, S, Q\}$ is an ideal kernel system then $Q$ is a Hellinger integral over $E$ in the sense that if $f, g$ is in $S \times S$ and $c>0$ there is a finite sequence $s$ in $E$ such that if $t$ is a finite sequence in $E$ of which $s$ is a subsequence then $\left|Q(f, g)-Q\left(f, P_{t} g\right)\right|<c$. NOTE: in the ideal system $\{K,[0,1], S, Q\}$ of part I (above), if $t$ is a subdivision of $[0,1]$ then $Q\left(f, P_{t} g\right)$ is the corresponding approximating sum for the integral $Q(f, g)=Q_{0}(f[0]$, $\left.H^{-1} g[0]\right)+\int_{0}^{1} Q_{0}\left(d f,[d \phi]^{-1} d g\right)$. (Received July 28,1955 .)

732t. J. S. MacNerney: Hellinger integrals in inner product spaces. V.

This is an application of parts I-IV to integral equations. Let $S$ be the linear space of all functions $f$ from $[0,1]$ to $\left\{S_{0}, Q_{0}\right\}$ such that $\int_{0}^{1} Q_{0}\left(d f,[d \phi]^{-1} d f\right)$ exists. A study is 
made of connections between $S$ and a system of Stieltjes (mean) integral equations: (*) $f(b)-f(a)+\int_{a}^{b} d \phi \cdot g=0$ and $\int_{a}^{b} d \theta \cdot f+g(b)-g(a)=h(b)-h(a)$ for each subinterval $[a, b]$ of $[0,1]$, where $h$ is a function of bounded variation from $[0,1]$ to $\left\{S_{0}, Q_{0}\right\}$. With suitable restrictions on $\phi$ and $\theta$ (which are nondecreasing functions from $[0,1]$ to $\left.B\left\{S_{0}, Q_{0}\right\}\right)$, the equation $Q(f, g)=\int_{0}^{1} Q_{0}\left(d f,[d \phi]^{-1} d g\right)+\int_{0}^{1} Q_{0}(f, d \theta \cdot g)$ defines an inner product $Q$ for $S$ and there is a kernel in the space $\{S, Q\}$. Various boundary value problems connected with the system $\left(^{*}\right)$ determine certain subspaces of $\{S, Q\}$, and lend themselves to classification in terms of the relationships among the associated subspaces; the boundary condition $g(0)=g(1)=0$ determines the whole space. These boundary value problems lead to integral equations, of the form $f(s)-z$ - $\int_{0}^{1} K(s, u) \cdot d \Psi(u) \cdot f(u)=g(s)$ with $\Psi$ of bounded variation from $[0,1]$ to $B\left\{S_{0}, Q_{0}\right\}$, which resemble those arising in the classical Sturm-Liouville theory of second order differential equations with two-point boundary conditions. (Received July 28, 1955.)

\section{3t. J. S. MacNerney: Hellinger integrals in inner product spaces.} VI.

This is an application of parts II-IV to function theory. Let $E$ be a connected domain in the number plane, and $P$ be a weakly continuous function from $E$ to $B\left\{S_{0}, Q_{0}\right\}$ such that, for each closed bounded set $D$ lying in $E, Q_{0}(x, P[z] x)$ is real and has positive upper and lower bounds for $z$ in $D$ and $Q_{0}(x, x)=1$. The function $f$ from $E$ to $\left\{S_{0}, Q_{0}\right\}$ is regular provided that for each $x$ in $S_{0}$ the function $g, g(z)$ $=Q_{0}(f[z], x)$ for $z$ in $E$, is a regular (analytic) function in $E$. Let $S$ be the set to which $f$ belongs only in case $f$ is a regular function from $E$ to $\left\{S_{0}, Q_{0}\right\}$ and the real function $Q_{0}(f, P f)$ is integrable over $E:(1) S$ is a linear space; (2) $Q(f, g)=\iint_{E} Q_{0}(f, P f)$ defines an inner product $Q$ for $S$; (3) the inner product space $\{S, Q\}$ is complete and there is a kernel in $\{S, Q\} ;(4)$ if the condition that $P$ be weakly continuous is replaced by any condition which ensures (1), then (2) and (3) still hold. An example is given, with $E$ the interior of the unit circle and $\left\{S_{0}, Q_{0}\right\}$ the 2-dimensional complex Euclidean space, wherein the only $2 \times 2$ constant matrices which belong to $B\{S, Q\}$ as "constant multipliers" are those matrices $A$ such that $A_{11}-A_{22}=A_{12}-A_{21}: P(z)_{11}=[1-|z|]$, $P(z)_{12}=P(z)_{21}=\left[1-|z|^{2}\right] / 2$, and $P(z)_{22}=\left[1-|z|^{3}\right] / 3$. (Received July 29, 1955.)

\section{H. W. Milnes: Convexity of Orlicz spaces.}

Let $L_{\Phi}$ be the Orlicz space over $\Delta=[a, b]$ generated by an increasing function $0 \leqq \phi(u) \leqq+\infty$ with inverse $\psi(v)$ and respective integrals $\Phi(u), \Psi(v)$, and the norm defined by $\|f\|=\sup \int_{\Delta}|f(x)| g(x) d x$ (supremum taken for all $g(x) \geqq 0$ with $\int_{\Delta} \Psi(g) d x$ $\leqq 1$ ). Conditions for strict and uniform convexity of $L_{\Phi}$ are obtained. $L_{\Phi}$ is strictly convex (i.e. boundary of the unit sphere in $L_{\Phi}$ does not contain segments) if and only if $\psi(v)$ and $\Psi(v)$ are continuous. $L_{\Phi}$ is uniformly convex if and only if in addition (i) $\lim \inf [\phi(u+\epsilon u) / \phi(u)]>1$ for all $\epsilon>0$, (ii) lim sup $[\Phi(2 u) / \Phi(u)]<+\infty$. Essential for the proofs is the fact that when $\lim _{v \rightarrow \infty} \psi(v) \rightarrow \infty$ for each $f \in L_{\Phi}$, there is a $g(x)$ with the properties $\int_{\Delta} \Psi(g) d x=1, \psi(g(x))=$ Const. $|f(x)|$ such that $\|f\|=\int_{\Delta}|f(x)| g(x) d x$. (Received September 1, 1955.)

735t. C. J. Neugebauer: A strong cyclic additivity theorem of a surface integral.

Let $X$ be a compact subset of $E_{3}$ (Euclidean 3-space), and let $T$ be a continuous mapping from the unit square $Q$ of $E_{2}$ (Euclidean 2-space) into $E_{3}$ such that $T(Q) \subset X$ and $L(T)<\infty$, where $L(T)$ is the Lebesgue area of the surface $S$ represented by $T$. 
For such a continuous mapping, L. Cesari has introduced a surface integral $J(T)$ $=(S) \int F d \sigma$ as a Weierstrass integral (Ann. Scuola Norm. Sup. Pisa (2) vol. 13 (1944) pp. 77-117). If $T=l m, m: Q \Rightarrow M, l: M \rightarrow E_{3}$ is a monotone-light factorization of $T$, then $\mathrm{J}$. Cecconi has shown that $J(T)$ is weakly cyclicly additive in the following sense. If $r_{C}$ denotes the monotone retraction from $M$ onto a proper cyclic element $C$ of $M$, then $J(T)=\sum J\left(l r_{C} m\right), C \subset M$. This result can be extended by using the concept of an unrestricted factorization of $T$ due to E. J. Mickle and T. Radó (Trans. Amer. Math. Soc. vol. 66 (1949) pp. 347-365). Let $T=s f, f: Q \rightarrow M, s: M \rightarrow E_{3}$ be an unrestricted factorization of $T$. If $r_{C}$ has the same meaning as above and if $\sum^{\prime}$ denotes the summation over all proper cyclic elements $C$ of $M$ for which $f(Q) \cap C \neq \phi$, then $J(T)$ is strongly cyclicly additive, i.e., $J(T)=\sum^{\prime} J\left(s r_{C} f\right)$. This formula also extends a strong cyclic additivity theorem of $L(T)$ due to T. Rad6. Extensions are available. (Received July 14, 1955.)

\section{Robert Osserman: On Ahlfors' counting function $n(t)$.}

The class of Riemann surfaces considered consists of simply-connected covering surfaces of the plane having only algebraic singularities over finite points. Fixing a point on the surface, we define $n(t)$ to be the total order of branch points whose distance along the surface to the fixed point is less than $t$. Ahlfors has proved (Comment. Math. Helv. vol. 3 (1931) pp. 173-177) that a sufficient condition for parabolic type is $\int^{\infty} d t / \operatorname{tn}(t)=\infty$. In the present paper further results are obtained relating the growth of $n(t)$ to the type of the surface. In particular, if all branch points of the surface project onto a bounded set in the plane, then the divergence of $\int^{\infty} d t / n(t)$ is already sufficient. On the other hand, if no further restrictions are made on the class of surfaces considered, then Ahlfors' condition is the best possible. In fact, a subclass of surfaces is exhibited for which Ahlfors' condition is both necessary and sufficient for parabolic type. This class includes examples of hyperbolic surfaces with $n(t)<t^{\epsilon}$ for arbitrarily small $\epsilon>0$. Finally, it is pointed out that no criterion for hyperbolic type can depend on $n(t)$ alone, since there exist parabolic surfaces with $n(t)$ arbitrarily large. (Received July $18,1955$.

\section{R. S. Pinkham: Inversion of the Laplace and Stieltjes trans-} forms utilising difference operators.

The paper is divided into four parts. Part I contains a new real inversion formula for the Stieltjes transform which utilizes differences of the generating function rather than derivatives. A generalized form of the asymptotic method of Laplace is developed and used to evaluate the singular integrals which appear. Part II deals with a new inversion formula for the Laplace transform which requires only knowledge of the generating function along the axis of reals. Part III presents the representation theory resulting from the operators of Parts I and II, while in Part IV an operator is constructed for solving the moment problem for the Stieltjes transform. (Received June $30,1955$.

\section{Pasquale Porcelli: Inversion of a Lebesgue-Stieltjes integral} equation.

The integrals in this paper are in the Lebesgue-Stieltjes sense. The following theorem is proved; Theorem: If each of $s(t)$ and $g(t)$ is a nondecreasing function on $[a, b]$ and $f(t)$ is a nonnegative $g$-summable on $[a, b]$ such that $s(a)=g(a)=0$ and (i) $s(u)=\int_{a}^{u} f(t) d g(t)$ for $a<u \leqq b$, then in order for $1 / f(t)$ to be $s$-summable and (ii) 
$g(u)=\int_{a}^{u}\{1 / f(t)\} d s(t)$ it is necessary and sufficient that $l_{g} R(f)=0$, where $R(f)$ is a subset of $[a, b]$ and $t$ is in $R(f)$ only if $f(t)=0$, and $l_{0} R(f)$ denotes the outer $g$-length of $R(f)$. (Received July 15, 1955.)

\section{9t. R. T. Prosser: On the representations of $W^{*}$ algebras.}

Let $H$ be a hilbert space, and $A, A^{\prime} W^{*}$ algebras of operators acting on $H$, where $A^{\prime}$ is the commutant of $A$. For each pair of cardinals $m, n$, zero or infinite, there exist a hilbert space $H_{m, n}$ and $W^{*}$ algebras $A_{m}$ and $A_{n}^{\prime}$ acting on $H_{m, n}$ such that $A_{n}^{\prime}$ is commutant of $A_{m}$, and: $A_{0}$ is finite, $A_{m}$ is either zero or cardinally homogeneous of degree $m$, for $m>0$; likewise for $A_{n}^{\prime} ; H$ is the direct sum of the $H_{m, n}, A$ is the direct sum of the $A_{m}$, and $A^{\prime}$ is the direct sum of the $A_{n}^{\prime}$. Moreover, there exist hilbert spaces $M_{m}, N_{n}$, and $P_{m, n}$, and $W^{*}$ algebras $B_{m}, C_{m}$ and $B_{n}^{\prime}, C_{n}^{\prime}$, such that; $M_{m}$ has dimension $m, N_{n}$ has dimension $n ; B_{m}$ consists of all operators acting on $M_{m}, B_{n}^{\prime}$ consists of all operators acting on $N_{n}, C_{m}$ and $C_{n}^{\prime}$ are strongly semi-simple algebras acting on $P_{m, n}$ with $C_{n}^{\prime}$ the commutant of $C_{m} ; H_{m, n}$ is the tensor product of $M_{m}, N_{n}$ and $P_{m, n}, A_{m}$ is the tensor product of $B_{m}$ and $C_{m}$, and $A^{\prime}$ is the tensor product of $B_{n}^{\prime}$ and $C_{n}^{\prime}$. The $H_{m, n}, A_{m}, A_{n}^{\prime}, M_{m}, N_{n}, B_{m}$ and $B_{n}^{\prime}$ are unique (up to unitary equivalence), and the possible choices for $P_{m, n}, C_{m}$ and $C_{n}^{\prime}$ may be described in terms of a suitable double dimension function. These results summarize the multiplicity theory for representations of general $W^{*}$ algebras. (Received July 18, 1955.)

\section{R. T. Prosser: On the structure of $W^{*}$ algebras.}

Let $A$ be any $W^{*}$ algebra. Then for each cardinal $n$, zero or infinite, there exists a $W^{*}$ algebra $A_{n}$ such that: $A_{0}$ is finite, $A_{n}$ is either zero or cardinally homogeneous of degree $n$, for $n>0$, and $A$ is the direct sum of the $A_{n}$. Moreover, there exist $W^{*}$ algebras $B_{n}$ and $C_{n}$ such that: $B_{n}$ is the full $n \times n$ matrix algebra, $C_{n}$ is strongly semisimple, and $A_{n}$ is the $W^{*}$ tensor product of $B_{n}$ and $C_{n}$. The $A_{n}$ and $B_{n}$ are unique (up to equivalence), and the possible candidates for $C_{n}$ may be described in terms of a suitable dimension function. In particular, if $A$ is of type I, each $C_{n}$ may be chosen abelian; if $A$ is of type II, each $C_{n}$ may be chosen finite; if $A$ is of type III, each $C_{n}$ is unique. These results summarize the known structure theorems for general $W^{*}$ algebras. (Received July 18, 1955.)

741t. M. O. Reade: A class of univalent functions. Preliminary report.

In a recent paper, W. Kaplan introduced the class of near-to-convex functions [Michigan Mathematical Journal vol. 1 (1952) pp. 169-185]. In this article, the present author uses Kaplan's new class to prove the following result. Let $f(z)$ $\equiv z+\sum_{2}^{\infty} a_{k} z^{k}$ be analytic and let $f^{\prime}(z) \neq 0$ for $|z|<1$. If $\int_{\theta_{1}}^{\theta_{2}} \operatorname{Re}\left[1+r e^{i \theta} f^{\prime \prime}\left(r e^{i \theta}\right) / f^{\prime}\left(r e^{i \theta}\right)\right]$ $d \theta>-\pi / 2$ for all $\theta_{1}<\theta_{2}$ and for all $0 \leqq r<1$, then $f(z)$ is univalent, and $\left|a_{n}\right| \leqq(n+1) / 2$ for $n=1,2,3, \cdots$. This includes results due to Robertson [Amer. J. Math. vol. 58 (1936) pp. 465-472] and Renyi [Publ. Mathem. Debrecen. vol. 1 (1949) pp. 18-23]. (Received May 11, 1955.)

742. W. T. Reid: A comparison theorem for self-adjoint differential equations of second order.

For $L_{\alpha}[y] \equiv\left(R_{\alpha}(t) y^{\prime}\right)^{\prime}+P_{\alpha}(t) y=0(\alpha=1,2)$, with $P_{\alpha}(t)$ real-valued continuous and $R_{\alpha}(t)$ positive functions of class $C^{\prime}$ on an interval $J$, and $T_{\alpha}^{+}\left(t_{0}\right)\left\{T_{\alpha}^{-}\left(t_{0}\right)\right\}$ the least \{greatest value of $t$ succeeding \{preceding $t_{0}$ and defining a focal point of $t=t_{0}$ 
relative to $L_{\alpha}(y)=0$ on $J$, the following comparison theorem is established: if $\left(R_{2} / R_{1}\right)^{\prime}$ $\geqq 0, P_{1} \geqq 0$ and $P_{2} / R_{2} \leqq P_{1} / R_{1}$ on $J$, then the existence of $T_{2}{ }^{+}\left(t_{0}\right)$ implies that $T_{1}{ }^{+}\left(t_{0}\right)$ exists and $T_{1}{ }^{+}\left(t_{0}\right) \leqq T_{2}{ }^{+}\left(t_{0}\right)$; similarly, if $\left(R_{2} / R_{1}\right)^{\prime} \geqq 0, P_{2} \geqq 0, P_{1} / R_{1} \leqq P_{2} / R_{2}$ then the existence of $T_{1}^{-}\left(t_{0}\right)$ implies that $T_{2}^{-}\left(t_{0}\right)$ exists and $T_{1}^{-}\left(t_{0}\right) \leqq T_{2}{ }^{+}\left(t_{0}\right)$. Various consequences of this result are given. In particular, it is shown that this result implies a comparison theorem on conjugate points for equations $\left(r(x) u^{\prime}(x)\right)^{\prime}+r(x) u(x)=0, x \in I_{x}$, and $\left(s(y) v^{\prime}(y)\right)^{\prime}+s(y) v(y)=0, y \in I_{y}$, that has been obtained by H. F. Weinberger using the maximum property of Cauchy's problem for hyperbolic partial differential equations. In addition for a differential equation $\left(R(t) y^{\prime}\right)^{\prime}+P(t) y=0$, with $R(t), P(t)$ positive functions of class $C^{\prime}$ on $[a, \infty)$, there are obtained certain criteria for oscillation and nonoscillation. (Received July 18, 1955.)

\section{3t. I. F. Ritter: Computation of quadratic factors of a polynomial.}

The first part of this article describes a new algorithm for the approximate computation of the coefficients $a, b$ in a quadratic factor $\left(z^{2}-a z-b\right)^{m}$, of multiplicity $m$, of a polynomial. It is shown that the procedure is equivalent to the Bernoulli-Euler method, but avoids the explicit computation of the recurrent sequence of that method. This has the advantage that the difficulty of scaling the numbers in the recurrent sequence does not arise. The process also explains the convergence behavior of the iteration of S-N. Lin by bringing out that the Lin process anticipates the monotonic convergence of the Bernoulli-Euler method. In the second part the Lin process is generalized to quadratic factors of any multiplicity $m$ and supplied with practical tests for finding $m$ and for convergence. For dealing with cases in which the Lin process diverges, the Newton iteration given by Bairstow is extended to any $m$. Although this process is generally applicable, a similar and equally practical higher order iteration is finally described that converges faster than the Bairstow method whenever the Lin iteration would converge. (Received July 18, 1955.)

\section{4t. D. W. Robinson: A note on the definition of a matric function.}

Let $T$ be a finite square matrix over the field of complex numbers, with distinct eigenvalues $\lambda^{1}, \cdots, \lambda^{k}$, and corresponding projections (Frobenius covariants) $E^{1}, \cdots, E^{k}$. Let $F[T]$ be the class of all complex valued functions of a complex variable that are analytic on an open set $N$ containing the eigenvalues of $T$. A definition (proposed by E. Cartan in a letter to G. Giorgi) of a matrix value corresponding to $T$ under $f \in F[T]$ is (1) $(2 \pi i)^{-1} \cdot \int_{C}(z I-T)^{-1} f(z) d z$, where $C=\sum_{i=1}^{k} C^{i}, C^{i}$ being a simple closed curve in the neighborhood $N^{i} \subset N$ of $\lambda^{i}$ and enclosing $\lambda^{i}$. The following theorem is proved: For every $T$ and every $f \in F[T]$ let there correspond a matrix $f(T)$. Then $f(T)$ is given by (1) above if and only if this correspondence satisfies either of the following sets of conditions: (1.1) The combinatorial requirements of Fantappiè, and, (1.2) If $f, f_{n} \in F[T],\left\{f_{n}\right\}$ analytic in and converging to $f$ on $N^{i}$, uniformly on every compact subset of $N^{i}$, then $E^{i} f_{n}(T)$ converges and $E^{i} f(T)=\lim E^{i} f_{n}(T)$; or, (2.1) If each eigenvalue of $T$ is of index one then $f(T)=\sum_{i=1}^{k} E^{i} f\left(\lambda^{i}\right)$, and, (2.2) If $\left\{T_{n}\right\}$ is a sequence of matrices such that $f \in F\left[T_{n}\right]$ for all $n$ and $\lim T_{n}=T$, then $\left\{f_{n}(T)\right\}$ converges and $f(T)=\lim f\left(T_{n}\right)$. (Received July 15, 1955.)

745t. L. B. Robinson: On tensors and covariants of differential systems.

The author has computed a complete system of tensors associated with differential systems. They are given by the formula $I_{i j}=(1 / M) \sum_{k=1}^{3} \sum_{l=1}^{3} M_{i j}^{k l} \Psi_{k l}$ where the $\Psi$ are arbitrary functions of the covariants. Now reduce the covariants to canonical form. If the $\Psi$ are rational functions it is then easily seen that they are rational func- 
tions of the fundamental covariants. If the $\Psi$ are polynomials they are polynomial functions of the fundamental covariants. So the system of tensors is functionally, rationally, and algebraically complete. This is what Gordan proved for the covariants of binary forms. (Received May 6, 1955.)

\section{F. Virginia Rohde: A note on partial fractions.}

In cases where the study of partial fractions is left until the calculus it would seem worth while to use methods of the calulus in determining the coefficients. For all cases of linear factors in the denominator these coefficients can be expressed in terms of the derivatives of the product of the function and the highest power of the given factor evaluated at the zero of the factor in much the same manner as is done in the determination of residues in the theory of complex variables. (Received July 18, 1955.)

\section{7t. L. A. Rubel: Sets of positive integers of upper density one.}

This paper is concerned with several of the most useful upper density functions. The main result is that the class of sets of positive integers of upper density one is the same for each of these upper density functions. For sets $A$ of positive integers, the author defines (1) the upper asymptotic density, $\bar{D}(A)=\lim \sup _{(t \rightarrow \infty)} A(t) / t$ where $A(t)$ denotes the number of integers $n$ in $A$ such that $n \leqq t ;(2)$ the upper mean density, $\bar{D}_{M}(A)=\lim \sup _{(t \rightarrow \infty)} t^{-1} \int_{0}^{t} A(x) / x d x$; (3) the upper Abel density, $\bar{D}_{a}(A)=\lim \sup _{(t \uparrow 1)}(1-t) \sum_{n \in A} t^{n} ;(4)$ the upper Poisson density, $\bar{D}_{P}(A)=2 / \pi \lim$ $\sup _{(t \rightarrow \infty)} \int_{0}^{\infty}(A(y) / y) t\left(t^{2}+y^{2}\right)^{-1} d y$; (5) the modified upper logarithmic density, $\bar{D}_{L}(A)=\inf _{(\lambda>1)} \lim \sup (x \rightarrow \infty)\left\{(\log \lambda)^{-1} \phi(x, \lambda)\right\}$ where $\phi(x, \lambda)=\sum 1 / n$, the sum being taken over all $n$ in $A$ for which $x \leqq n \leqq \lambda x$. The main theorem states: If, for a given set $A$ of positive integers, any one of the numbers $\bar{D}(A), \bar{D}_{M}(A), \bar{D}_{a}(A), \bar{D}_{P}(A), \bar{D}_{L}(A)$ is equal to 1 , then all of these numbers are equal to 1 . The proof depends principally upon the author's optimal extension of Carlson's theorem on entire functions (Bull. Amer. Math. Soc. Abstract 61-3-417). This extension states that for each set $A$ of positive integers, each entire function $f(z)$ satisfying the conditions (i) $f(n)=0$ for each $n$ in $A$, (ii) $f(z)=O(1) \exp \tau|z|$ for some $\tau<\infty$, (iii) $f(i y)=O(1) \exp c|y|$ for some $c<\pi$ must vanish identically if and only if $\bar{D}(A)=1$. In view of the present paper, we may replace the condition $\bar{D}(A)=1$ by any one of the conditions $\bar{D}_{L}(A)=1, \bar{D}_{P}(A)=1, \bar{D}_{a}(A)=1$, $\bar{D}_{M}(A)=1$. (Received June 27,1955 .)

748t. Walter Rudin: An algebraic characterization of conformal equivalence.

Let $A(R)$ be the algebra whose members are the single-valued regular analytic functions on the open Riemann surface $R$. Theorem: If $A\left(R_{1}\right)$ and $A\left(R_{2}\right)$ are algebraically isomorphic, then $R_{1}$ and $R_{2}$ are conformally equivalent (the converse is obvious). The proof depends on the fact that for every $p \in R$ there is an $f \in A(R)$ which has a simple zero at $p$ and vanishes nowhere else (see Florack, Schr. Math. Inst. Münster, no. 1, 1948). It follows that every principal maximal ideal of $A(R)$ consists of the functions vanishing at some point of $R$. If $T$ is an isomorphism of $A\left(R_{2}\right)$ onto $A\left(R_{1}\right)$, there is therefore a one-to-one mapping $t$ of $R_{1}$ onto $R_{2}$ such that $(T f)(p)$ $=f(t(p))$ for all $p \in R_{1}$ and all $f \in A\left(R_{2}\right)$; this implies that $t$ is conformal. (Received July $25,1955$.

749. Walter Rudin: The automorphisms and endomorphisms of the group algebra of the unit circle.

Theorem I. For any mapping $t$ of the set $J$ of all integers into itself, the statements 
(A) and (B) are equivalent: (A) If $\sum_{-\infty}^{\infty} c(n) e^{i n \theta}$ is the Fourier series of a function $f \in L_{1}$, then $\sum_{-\infty}^{\infty} c(t(n)) e^{i n \theta}$ is the Fourier series of a function $T f \in L_{1}$. (B) There exists a sequence $\{s(n)\}$ and a positive integer $p$ such that (i) $t(n)=s(n)$ except possibly for a finite set of values of $n$; (ii) $s(n+p)+s(n-p)=2 s(n)$ for every $n$; (iii) $s(n+p)$ $\neq s(n)$ for every $n$. The fact that (B) implies (A) is a matter of simple computation; the proof of the converse depends on the observation that, if (A) holds, then the sequences $\left\{e^{i x t(n)}\right\}$ are, for each real $x$, sequences of Fourier-Stieltjes coefficients of measures $\mu_{x}$ such that $\mu_{x *} \mu_{y}=\mu_{x+y}$. Theorem II. If $t$ is a one-to-one mapping of $J$ onto itself which satisfies (B), then $T$ is an automorphism of the convolution algebra $L_{1}$ on the unit circle. Every automorphism of $L_{1}$ is obtained in this manner. Theorem III. If $t$ satisfies (B) and if $N$ is a set of integers which differs from a periodic set by only a finite number of elements, then the mapping which assigns to the series $\sum_{-\infty}^{\infty} c(n) e^{i n \theta}$ the series $\sum_{n \in N} c(t(n)) e^{i n \theta}$ defines an endomorphism of $L_{1}$. Every endomorphism of $L_{1}$ is obtained in this manner. (Received June 10,1955.)

\section{E. C. Schlesinger: Conformal invariants and prime ends.}

A sequence of points $\left\{a_{n}\right\}$ of points of a simply connected plane region $G$ "converges to a prime end of $G^{\prime \prime}$ if $\lambda(\Gamma)=0$. Here $\Gamma$ is the family of cross-chains (=union of a finite number of connected crosscuts of $G$ ) which separate (in $G$ ) all but a finite number of the $a_{n}$ from $E ; E$ is a closed subset of $G$ which contains at most a finite number of the points $a_{n}$; finally, $\lambda(\Gamma)$ is the extremal length (L. Ahlfors and A. Beurling, Acta Math. vol. 83 (1950) pp. 101-129) of the family $\Gamma$. Two sequences of points of $G$ "converge to the same prime end of $G$," if the mixed sequence formed from them converges to a prime end of $G$. These formulations do not depend on the set $E$. The "prime ends of $G$ " are the equivalence classes (of sequences of points) obtained in this way. Theorem: The above definition is equivalent to that of Caratheodory (Math. Ann. vol. 73 (1913) pp. 323-370). Theorem: If $\left\{a_{n}\right\}$ is a sequence of points of $G$ converging to the prime end $\pi$ of $G$ then all limit points of the sequence are "principal" on $\pi$ (Carathéodory, op. cit. pp. 353-354), provided the sequence $\left\{\lambda_{n}+\lambda_{n}^{-1}\right\}$ remains bounded, where $\lambda_{n}$ is the extremal length of a certain family of crosscuts determined by the region $G$, the prime end $\pi$, and the point $a_{n}$. The second of these theorems is a conformally invariant and intrinsic form of a result due to Lindelöf (Acta Soc. Sci. Fenn. vol. 46 (1915) No. 4, p. 28) on "angular" approach. (Received July 8, 1955.)

\section{1t. I. E. Segal: Note on induced representations.}

The need for quasi-invariant measures and certain measurability considerations in the development of the notion of induced representation of a locally compact group are eliminated in a new approach, which also makes possible the extension of the basic theory to not necessarily separable groups. The method uses abelian operator rings. (Received July 19, 1955.)

752t. George Seifert: $A$ note on a result concerning limit cycles for an autonomous system.

Consider the system $\dot{x}=y, \dot{y}=-g(x)-f(x, y) y$, where $g$ and $f$ are continuous and satisfy Lipschitz conditions in every bounded domain in the $(x, y)$ plane, $x g(x)>0$ for $x \neq 0$, and $\int_{0}^{ \pm \infty} g(x) d x=+\infty$. It has been shown by A. DeCastro (Boll. Un. Mat. Ital. (3) vol. 9 (1954) pp. 280-281) that if this system has a periodic solution whose trajectory, a cycle, will be denoted by $C$, then the system $\dot{x}=y, \dot{y}=-g(x)-f_{1}(x, y) y$ will have a periodic solution whose cycle $C_{1}$ is interior to $C$ provided (i) $f_{1}(0,0)<0$ and 
(ii) $f(x, y)<f_{1}(x, y)$ for all $x$ and $y$. It is observed here that this same result may be obtained without the hypothesis $\int_{0}^{ \pm \infty} g(x) d x=+\infty$, and under a condition weaker than (ii); namely, $f(x, y) \leqq f_{1}(x, y)$ on $C$, the equality holding there for at most a finite number of points. (This research was supported by the United States Air Force through the Office of Scientific Research of the Air Research and Development Command). (Received July 19, 1955.)

\section{3t. V. L. Shapiro: Localization of Laplace series.}

Let $T=\sum_{n=1}^{\infty} Y_{n}(x)$ be a series of surface spherical harmonics where $Y_{n}(x)$ $=O\left(n^{w}\right), w \geqq 0$, uniformly for $x$ on $S$, the surface of the unit sphere in three-dimensional Euclidean space. To $T$ can then be associated a function $F(x)=\sum_{n=1}^{\infty}(-1)^{k}$ - $Y_{n}(x) /[n(n+1)]^{k}$ where $k$ is the smallest integer greater than $(w+1) / 2$. It is then shown in this paper that if $F(x)$ is of class $C^{(2 k)}$ on a domain $D$ of $S$, then $T$ is Abel summable to $\Delta^{k} F(x)$ uniformly in every closed domain contained in the interior of $D$, where $\Delta^{k}=\Delta\left(\Delta^{k-1}\right)$ and $\Delta$ is the Laplacian on the surface of the sphere. Another localization theorem of a slightly different nature also obtained in this paper is the following: Let $F(x)$ be a function integrable on $S$ and let $T[F]$ designate the Laplace series of $F$. Let $\Delta T[F]$ be the series of surface spherical harmonics obtained by formally applying the Laplacian operator to $T$. Suppose $F(x)$ has a generalized Laplacian at the point $x_{0}$ equal to $p$. Then $\Delta T[F]$ is Abel summable to $p$ at the point $x_{0}$. This latter result can also be stated in the following manner: Given a series of surface spherical harmonics $T=\sum_{n=1}^{\infty} Y_{n}(x)$ such that $\sum_{n=1}^{\infty} Y_{n}(x) / n(n+1)$ is the Laplace series of an integrable function. Then the Riemann summability of $T$ implies the Abel summability of $T$. (Received May 17, 1955.)

\section{4t. Paul Slepian: Theory of Lebesgue area of continuous maps of} 2-manifolds into $n$-space.

Let $X$ be a finitely triangulable topological space and let $f$ be a continuous function on $X$ into Euclidean $n$-space, $E_{n}$, with $n \geqq 2$. For any integers $i$ and $j$ with $1 \leqq i$ $<j \leqq n$, let $P_{n}^{i, j}$ be the projection of $E_{n}$ onto $E_{2}$ defined by $P_{n}^{i, j}(x)=\left(x_{i}, x_{j}\right) \in E_{2}$ for each $x=\left(x_{1}, x_{2}, \cdots, x_{n}\right) \in E_{n}$. Let $L_{2}$ be 2-dimensional Lebesgue area. Federer has shown (Ann. of Math. vol. 61 (1955) p. 289) that if $X$ is a subset of the plane, then $L_{2}(f) \leqq \sum_{1 \leqq i<j \leqq n} L_{2}\left(P_{n}^{i, i} \circ f\right)$. The principal result of this paper states that the above inequality remains true if $X$ is a compact 2-manifold (with or without boundary). This inequality is of fundamental importance for the extension of the whole theory of Lebesgue area to continuous maps of 2 -manifolds into $n$-space. (Received June 3, 1955.)

\section{5t. D. B. Sumner: A generalization of the averaging operator.}

The averaging operator $\nabla f(z)=[f(z+h)+f(z)] / 2$ has been studied by Nörlund and certain aspects of its integer powers by Milne-Thomson. The operator $\nabla^{\lambda}$ is defined for any real $\lambda$, and is shown to be applicable to a wide class of functions. Generalized Euler polynomials associated with the operator are defined, and some of their more important properties established; and the inversion theorem for the functional equation $\nabla f(z)=\phi(z)$ is proved. (Received July 5,1955 .)

756t. D. B. Sumner: Generalized powers of the difference operator. Preliminary report.

A generalized power $\Delta^{\lambda}$ of the difference operator $\Delta f(z)$ is defined and its more im- 
portant properties established, including those of the generalized Bernoulli polynomials associated with it. An inversion theorem for the functional equation $\Delta f(z)$ $=\phi(z)$ is proved; and eigenvalues and eigenfunctions of the operator are discussed. (Received July 5, 1955.)

\section{W. C. Swift: An iterated matrix method of summability.}

Given a matrix $A=\left(a_{n k}\right)$, a sequence $\left\{x_{i}\right\}$ is said to be summable- $A$ to $x$ if one has $x=\lim _{k} \lim _{3} \sum_{i=1}^{i} a_{k i} x_{i}$. If, in this last expression, one or both of the limiting operations are themselves replaced by matrix summability methods, then the resulting transform is referred to as an iterated matrix method of summability. In general, the set of iterated matrix operators is generated recursively by replacing the natural limiting operations as they appear in conventional matrix summability by previously determined iterated matrix operators. If, from a fixed regular matrix $A$, it is possible to generate in this fashion an operator which sums a given sequence, then the value obtained is uniquely determined; and the sequence is said to be ultimately summable- $A$ to that limit. For the usual method of summability, Okada (Math. Zeit. vol. 23, pp. 62-71) has proved a theorem concerning the effectiveness for summing a Taylor's series to its analytic continuation. A generalization of this theorem for the iterated methods is found to be valid. As a result, it can be shown that for a very simply derived class of regular matrices, the sequence of partial sums of a Taylor's series is ultimately summable to its analytic continuation throughout the principal star domain of the function. (Received July 18, 1955.)

\section{T. T. Tanimoto: Minimal paths through $n$ points.}

Given $n$ points $P_{1}, P_{2}, \cdots, P_{n}$ in Euclidean space $E_{r}$ of dimension $r$, the following method is applied to determine minimal paths passing through each of the $n$ points: Let $A$ be the matrix consisting of elements $a_{i j}=a_{3 i}=d\left(P_{i}, P_{1}\right)>0$ for $i \neq j$, and $a_{i i}=0$, where $d\left(P_{i}, P_{j}\right)$ is the undirected distance between $P_{i}$ and $P_{j}$; and let $S$ be the associated doubly stochastic matrix consisting of elements $s_{i j}=s_{j i}=1 /(n-1)$, $i \neq j, s_{i i}=0$. If $X$ is the set of row vectors and $Y$ the column vectors of $S$, it is shown that $A$ considered as a two-person zero sum game has the solution $X_{i_{0}}, Y_{j_{0}},\left(2 /(n-1)^{2}\right)$ - $\left[T-W_{i_{0} i_{0}}\right]$ where $X_{i_{0}}$ is the vector with $1 /(n-1)$ as every component except for a zero in the $i_{0}$ th component, and similarly for $Y_{j_{0}}, T=(1 / 2) \sum_{i j} a_{i j}$, and $W_{i j}=(1 / 2)$ $\cdot\left(\sum_{\alpha} a_{\alpha i}+\sum_{\beta} a_{j \beta}-a_{i j}\right)$. The first moves of the game and the subgames determined by deleting the points of order two are played and the weight of a segment $\left(P_{i}, P_{j}\right)$ viz., $W_{i j}$ is used to determine optimal segments. The restrictions of the linear graphs desired are imposed and minimal paths are determined. (Received July 18, 1955.)

759. W. J. Thron: Analytic solutions of the functional equation $f(f(z))=g(z)$.

Using a theorem of Pólya (J. London Math. Soc. vol. 1 (1926) pp. 12-15) and Hadamard's factorization theorem it is proved that the functional equation $f(f(z))$ $=g(z)$, where $g(z)$ is an entire function of finite order, which is not a polynomial, and which takes on a certain value $p$ only a finite number of times, does not have a solution which is an entire function. (Received July $15,1955$. )

\section{0t. W. R. Utz: A third order differential equation.}

The following theorem is proved. Let $f(x)$ be a real differentiable function such that $\lim _{x \rightarrow \infty} f(x)=c, 0<c<\infty$, and such that $f^{\prime}(x)$ is bounded as $x \rightarrow \infty$. Let $p(x)$ be a real polynomial and let $a>0$ be a real number such that $p(a)=0, p^{\prime}(a)>0$. If $y=y(x)$ 
is a solution of $f(x) y^{\prime \prime \prime}-y y^{\prime \prime}+p\left(y^{\prime}\right)=0$ valid for large values of $x$ and for which $\lim _{x \rightarrow \infty} y^{\prime}(x)=a$, then $y=a x+b$, where $b$ is a constant. This theorem generalizes a theorem of G. H. Hardy (Proc. Cambridge Philos. Soc. vol. 35 (1939) pp. 652, 653) in which $f(x)=1, p\left(y^{\prime}\right)=2\left({y^{\prime}}^{2}-1\right), a=1$. Hardy's theorem was suggested by a paper of S. Goldstein (Proc. Cambridge Philos. Soc. vol. 35 (1939) pp. 338-340) who uses the equation in his study of the boundary layer for a steady two-dimensional flow of a fluid of constant density and constant kinematic viscosity. (Received July 19,1955.)

761t. S. E. Warschawski: Distortion in conformal mapping of variable domains. I. Estimates for the $L_{p}$ mean.

Let $C_{i}(i=1,2)$ be closed Jordan curves possessing continuous tangents, let $\beta_{i}(\sigma)$ be moduli of continuity of the tangent angles, $\tau_{i}(s)$, as functions of the arclength $s$. Suppose: (i) $C_{i}$ are contained in the ring $0<d \leqq|w| \leqq D$; (ii) if $\Delta s$ is the (shorter) arc of $C_{i}$ between $w^{\prime}$ and $w^{\prime \prime}$ then $\left(\Delta s /\left|w^{\prime}-w^{\prime \prime}\right|\right) \leqq c$; (iii) for some fixed $\epsilon>0, C_{2}$ is in the $\epsilon$-neighborhood of $C_{1}$, i.e. every point of $C_{2}$ is contained in a circle of radius $\epsilon$ about some point of $C_{1}$; (iv) let $\tau_{i}{ }^{*}(w)$ denote the tangent angle at $w$ on $C_{i}$; there exists an $\eta$ such that, for any $w_{2} \in C_{2}$, there is a point $w_{1} \in C_{1}$, with $\left|w_{2}-w_{1}\right| \leqq \epsilon$, such that $\left|\tau_{1}^{*}\left(w_{1}\right)-\tau_{2}^{*}\left(w_{2}\right)\right| \leqq \eta, 0<\eta \leqq \pi / 2-\alpha<\pi / 2$. Suppose $w=f_{i}(z)$ maps $|z|<1$, conformally onto the interior of $C_{i}$ such that $f_{i}(0)=0, f_{i}^{\prime}(0)>0$. Then it is proved: Under the assumptions (i), (ii), (iii), (iv) there exist, for every $p>1$, (1) an $M_{p}$ such that $\mathfrak{M}_{p}\left\{f_{1}-f_{2}\right\}$ $=\left\{(1 / 2 \pi) \int_{0}^{2 \pi}\left|f_{1}\left(e^{i \theta}\right)-f_{2}\left(e^{i \theta}\right)\right|^{y} d \theta\right\}^{1 / p} \leqq M_{p} \epsilon ;$ (2) a $K_{p}$ such that $\mathfrak{M}_{p}\left\{f_{1}^{\prime}-f_{2}^{\prime}\right\} \leqq K_{p}[\eta$ $\left.+\beta_{1}^{*}\left(2 c M_{p} \epsilon\right)\right]$, where $\beta_{1}^{*}(\sigma)$ is a convex majorant of $\beta_{1}(\sigma)$. The constants $M_{p}, K_{p}$ depend on $p, d, D, c, \alpha$ and the $\beta_{i}$ only. This contains an earlier result for $\beta_{1}(\sigma)=H \sigma^{\gamma}, 0<\gamma \leqq 1$, $\eta=q \epsilon^{\gamma}$ (Archiv für Mathematik vol. 4 (1955) pp. 102-114). (Received July 18, 1955.)

$762 t$. S. E. Warschawski: Distortion in conformal mapping of variable domains. II. Uniform estimates.

Using the notations of part I (preceding abstract) define: $\omega(\delta)=\operatorname{Max}_{i=1,2} \int_{0}^{\delta} \sigma^{-1}$

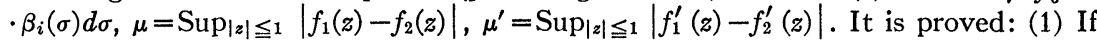
$\omega(\delta)<\infty$ and (i), (ii), (iii) of part I are satisfied, then $\mu \leqq M \in \log (1 / \epsilon)$. (2) If $\omega(\delta)<\infty$ and (i)-(iv) hold, then $\mu^{\prime} \leqq N\left\{\omega(\lambda \delta)+\left[\eta+\beta_{1}(2 c \mu)\right] \log (\pi / \delta)\right\}$ for every $\delta, 0<\delta<\pi / e$. The constants $M, N, \lambda$ depend on $d, D, c$, and the $\beta_{i}$ only. In particular, for $\beta_{1}=H_{1} \sigma$, $\beta_{2}=H_{2} \sigma^{\gamma}, 0<\gamma \leqq 1, \eta=q \epsilon, \delta=\epsilon^{1 / \gamma}$ one has $\mu^{\prime} \leqq N_{1} \in \log (1 / \epsilon)$, where $N_{1}=N_{1}(d, D, c$, $\left.H_{1}, H_{2}, \gamma, q\right)$. (3) Suppose that $C_{i}$ are represented by $w=w_{i}(t), 0 \leqq t \leqq a$, such that $0<b_{1} \leqq\left|w_{i}^{\prime}(t)\right| \leqq b_{2},\left|w_{i}^{\prime \prime}(t)\right| \leqq k\left(b_{1}, b_{2}, k\right.$ are constants) and that, for some $\epsilon>0$ and some $p>1, \mathfrak{M}_{p}\left\{w_{1}^{\prime \prime}(t)-w_{2}^{\prime \prime}(t)\right\} \leqq \epsilon$ and $\left|w_{1}(0)-w_{2}(0)\right| \leqq \epsilon$. Suppose, in addition, (i) and (ii) hold. If $\phi_{i}(w)$ denotes the inverse function of $f_{i}(z)$, then $\mid \phi_{1}^{\prime}\left(w_{1}(t)\right)$ $-\phi_{2}^{\prime}\left(w_{2}(t)\right) \mid \leqq \Lambda \epsilon, 0 \leqq t \leqq a$, where $\Lambda=\Lambda\left(d, D, c, b_{1}, b_{2}, k, p\right)$. This provides an estimate for the variation of the normal derivative of Green's function of first kind on the boundary under quite general deformations of the domain. (Received July 15, 1955.)

763t. A. D. Wasel: An initial value problem for mixed compressible fluid flows.

In order to study the stream function $\psi$ for compressible fluid flows, Bergman (Proc. Nat. Acad. Sci. U.S.A. vol. 29 (1943) p. 276) and Bers and Gelbart (Quarterly of Applied Mathematics vol. 1 (1943) p. 168) introduced solutions $\psi_{n j}, j=1,2$; $n=1,2, \cdots$, of $m(\psi) \equiv \psi_{H H}+l \psi_{\theta \theta}=0$, generated by an integral operator. Here $l=\left(1-M^{2}\right) / \rho^{2}$ ( $M$ the Mach number, $\rho$ the density), $H$ is a known function of the speed, and $\theta$ is the velocity direction. By modifying these solutions, the author con- 
structs solutions $\psi(H, \theta)=\left\{\sum_{n=1}^{\infty} n^{-1} a_{n-1}^{(2)}\left[\left(\begin{array}{c}n \\ 1\end{array}\right) \theta^{n-1} \int_{H_{0}}^{H} d H_{1}-3 !\left(\begin{array}{c}n \\ 8\end{array}\right) \theta^{n-3} \int_{H_{0}}^{H} d H_{3} \int_{H_{0}}^{H} z d H_{2}\right.\right.$ $\left.\left.\left.\cdot \int_{H 0}^{H_{2}} d H_{1}+\cdots\right]+\sum_{n=0}^{\infty} a_{n}^{(1)}\left[\theta^{n}-2 ! \sum_{2}^{n}\right) \theta^{n-2} \int_{H_{0}}^{H-1} d H_{2} \int_{H_{0}}^{H 2} l d H_{1}+\cdots\right]\right\}$ satisfying $\psi\left(H_{0}, \theta\right)$ $=\chi_{1}(\theta)$ and $\psi_{H}\left(H_{0}, \theta\right)=\chi_{2}(\theta)$ where $\chi_{k}(\theta), k=1,2$, are arbitrary analytic functions of $\theta$ possessing expansions $\sum_{n=0}^{\infty} a_{n}^{(k)} \theta^{n}, k=1,2$, respectively. (Received May 27,1955.)

764t. A. D. Wasel: An initial value problem for subsonic compressible fluid flows.

The author considers $s(\psi) \equiv \psi_{z^{\prime} \bar{z}^{\prime}}+N\left(\psi_{\mathbf{z}^{\prime}}+\psi_{\bar{z}^{\prime}}\right)=0$ (a form of $m(\psi)=0$, see preceding abstract, valid for subsonic flows) with $z^{\prime}=\lambda^{\prime}+i \theta^{\prime}, \bar{z}^{\prime}=\lambda^{\prime}-i \theta^{\prime}, \lambda^{\prime}=\lambda-\lambda_{0}, \lambda$ a conveniently chosen function of $M$, and $\lambda_{0}<0$. He defines $\psi$ to have a pole-like singularity at $z^{\prime}=\alpha$ with respect to the Bergman operator (Amer. J. Math. vol. 74 (1952) p. 444) generating solutions of $s(\psi)=0$, if the associate function $g\left(z^{\prime}\right)=\sum_{n=0}^{\infty} \mu_{n} z^{\prime n}$ of that operator has a simple pole at $z^{\prime}=\alpha$. He sets $\Psi^{*}\left(0, \theta^{\prime}\right)=\sum_{n=0}^{\infty} \beta_{n} \theta^{\prime \prime}$ and $\Psi_{\lambda}^{*}\left(0, \theta^{\prime}\right)$ $=\sum_{n=0}^{\infty} \gamma_{n} \theta^{\prime n}$, where $\Psi\left(\lambda^{\prime}, \theta^{\prime}\right)=\psi\left(z^{\prime}, \bar{z}^{\prime}\right)$ and $\Psi^{*}=H^{-1} \Psi, H$ being a known function of $\lambda^{\prime}$, and using Theorem 5.2 of the above reference he expresses $\mu_{n}$ in terms of $\beta_{n}, \gamma_{n}$, and certain constants depending on the coefficient $N$ of $s(\psi)=0$. Finally he shows that if $\alpha$ lies in the domain of validity of the operator and the coefficients $\beta_{n}$ and $\gamma_{n}$ are chosen in such a way that $\left|\left(\mu_{n+1} / \mu_{n}\right)-(1 / \alpha)\right|<k^{n}$ for all $n$ sufficiently large and $k<1$, then $\psi$ has a pole-like singularity at $z^{\prime}=\alpha$. (Received May 27, 1955.)

\section{R. E. Zindler: End points of trajectories.}

Given the system of equations $d x_{i} / d t=X_{i}\left(x_{1}, \cdots, x_{n}\right)$ where the $X_{i}$ are real continuous functions of the $x_{i}$ (real) in an open region $R$ except possibly at a closed set of singular points therein. A singular point is a point at which (a) all $X_{i}$ vanish, (b) one or more of them becomes infinite or, (c) the limit of any one of them is not independent of the direction of approach. It is shown that all trajectories (extensions of local solutions through nonsingular points by repeated application of the existence theorem) must end in singular points if they have a finite end point. (Received July $13,1955$.

\section{Applied Mathematics}

\section{R. J. Arms: Numerical calculation of the eigenvalues of matrix.}

With $A$ a square matrix and $x^{(0)}$ a fixed vector, we consider the sequence $\left\{x^{(n)}\right\}$ where $x^{(n)}=A^{n} x^{(0)}$. If $x_{i}^{(n)}$ is the $i$ th component of $x^{(n)}$ and $f_{i}(z)=\sum_{n=0}^{\infty} x_{i}^{(n)} z^{n}$, it is noted that $f_{i}(z)$ is a rational function whose denominator has the reciprocals of the eigenvalues of $A$ as roots. Hence, by theorems of Hadamard, Kronecker, Konig and others, there are algorithms for using the $x_{i}^{(n)}$ 's to compute the poles of $f_{i}(z)$. (Received July 7, 1955.)

767. R. C. F. Bartels (p) and A. C. Downing, Jr.: A note on the stability and convergence criteria for hyperbolic systems of equations.

The paper follows a method by Keller and Lax to establish criteria for the stability and convergence of the numerical solution of an initial value problem for hyperbolic systems. If the classical condition of Courant, Friedrichs and Lewy is satisfied, then the solution of the difference equations converges to the solution of the differential equations as $\bar{\Delta} x^{2} / \Delta t$ tends to zero. This result is obtained from an inequality giving a bound for a norm of the error in the solution in terms of bounds on the second derivatives of the solution. From this inequality, it is also possible to say something about an optimum choice of grid size. (Received July 18, 1955.) 
768. J. S. Bendat: Optimum time-variable filters for finite operating periods.

This paper presents a general solution to an integral equation characterizing optimum time-variable linear filters which operate from a finite starting point in time. Input signals are permitted to be arbitrary nonstationary random processes expressible in Fourier series ensemble form. The particular noise perturbation treated is represented by a nonstationary damped exponential-cosine autocorrelation function, which is of major importance in such applied fields as meteorology, radar and automatic control. A new technique is established for solving integral equations, involving Laplace Transform theory and sets of simultaneous time-varying equations. An asymptotic system design method is outlined for making concrete applications of the mathematical solutions. This practical procedure is illustrated with details of actual engineering systems. (Received July 5, 1955.)

\section{Garrett Birkhoff and J. B. Diaz (p): Non-linear networks.}

The main concern is with "connected networks," i.e. finite connected graphs consisting of a finite set of nodes (vertices), certain nodes being joined in pairs by a finite set of oriented links (branches), with the boundary (a certain subset of the set of nodes) supposed specified. In network flow problems, Kirchhoff's node law is supposed to hold at each interior node, while certain "boundary conditions" (of Dirichlet, Neumann or of mixed type) hold at boundary nodes. For physical equilibrium, the currents satisfy certain equilibrium relations: $i\left(a_{j}\right)=c_{j}\left(\Delta u_{j}\right)$ on each link $a_{j}$, where $\Delta u_{j}$ is the potential difference across the directed link $a_{j}$, and the "conductivity" functions $c_{j}$ are strictly increasing continuous functions of $\Delta u$. Uniqueness and existence theorems are proved for the various boundary value problems mentioned above. Under certain additional hypotheses regarding the conductivity functions, the existence of the solution of the mixed problem is shown by means of a constructive process of "exact point by point relaxation." (Received July 18, 1955.)

\section{J. W. Carr, III: Row-operation numerical inversion of high-} order matrices. Preliminary report.

An analysis is made of a so-called "row-operation procedure" for the determination of the inverse of a (nonsingular) nonsymmetric real matrix. This method is a logical extension of the procedure for nonsymmetric matrices discussed by $\mathrm{J}$. von Neumann and H. Goldstine, Numerical inverting of matrices of high order, Bull. Amer. Math. Soc. vol. 53 (1947) pp. 1021-1099. This algorithm is the equivalent of decomposition of an arbitrary nonsingular nonsymmetric matrix $A$ into the product $A=O T$, where $T$ is an upper-triangular matrix and $O$ an orthogonal matrix. The inverse $A^{-1}$ equals $T^{-1} O^{-1}$, and since the transpose of an orthogonal matrix is also its inverse, is obtained directly by row-operations alone, combined with determination of the maximum norms of the rows being operated on at any of the iterative stages of the procedure. Rearrangement of the rows in order of maximum norm similar to the "pivotal element technique," combined with successive row orthogonalizations, provides an inversion procedure stable under "round-off." The "row-operation procedure" requires the same number of multiplications $\left(2 n^{3}\right.$, where $n$ is the order of the matrix) as the von Neumann-Goldstine technique of pre-multiplication by the transpose, but is much easier to "code" for a high-speed electronic digital computer, because it deals only with additions of rows, multiplication of rows by constants, and row inner products. The final result obtained is the transpose of the inverse desired. (Received July 20,1955.) 


\section{R. V. Churchill: Generalized finite Fourier cosine transforms.}

Let $k_{n}(n=1,2, \cdots)$ denote the positive roots of the equation $h \cos k_{n}=k_{n} \sin k_{n}$ where $h$ is a constant other than zero. The integral transformation $T\{F\}=\int_{0}^{1} F(x)$ $\cdot \cos k_{n} x d x$ transforms $F^{\prime \prime}(x)$ into $-k_{n}^{2} f(n)-F^{\prime}(0)+\left[h F(1)+F^{\prime}(1)\right]$ cos $k_{n}$, where $f(n)=T\{F\}$. Further operational properties of the transformation are found, some in terms of certain dual transformations. The generalized Fourier series corresponding to the orthogonal functions $\cos k_{n} x$ furnishes a formula for the inverse transformation. The following convolution property of the transformation is established. If $f(n)$ and $g(n)$ are the transforms of two bounded integrable functions $F(x)$ and $G(x)$ on the interval $(0,1)$, if $F_{0}(x)$ denotes the even periodic extension of $F(x)$ with period 2, and if the asterisk denotes the convolution operation for the Laplace transformation, then under the transformation $T$ the image of the product $f(n) g(n)$ is the operation $2^{-1} \int_{0}^{1}\left[F_{0}(x+t)+F_{0}(x-t)\right] G(t) d t-h e^{-h x} * F(1-x) * G(1-x)$. Methods used here suggest procedures that may apply to other finite integral transformations whose kernels have no common period for all $n$. The results are applied to obtain a closed form of the solution of a boundary value problem. (Received July 18, 1955.)

772. C. L. Dolph and R. K. Ritt (p): On the Schwinger variational principle. Preliminary report.

The Schwinger variational principle for harmonic time dependent quantum mechanical scattering at arbitrary angles of reflection is formulated vigorously in a Hilbert space and its relation to more classical variational principles discussed. The completeness and validity of the approximation process is demonstrated in some special cases by use of the spectral operator theory of Dunford and Schwartz (Pacific Journal of Mathematics, September 1954). (Received July 19, 1955.)

773t. Jim Douglas, Jr.: On the errors in analogue solutions of heat conduction problems.

The approximate solution of heat conduction problems on analogue machines such as differential analyzers and resistance-capacitance circuits are most commonly obtained by replacing the space derivatives by finite differences, retaining the time derivative, and integrating the resulting system of ordinary differential equations on the machine. It has been conjectured by Hartree that the truncation error (as distinguished from various mechanical or electrical errors) is $O\left((\Delta x)^{2}\right)$, where $\Delta x$ is the largest space increment. This conjecture is affirmed for the quasi-linear parabolic equation $\Delta u=F\left(x_{1}, \cdots, x_{m}, t, u\right) u_{t}+G\left(x_{1}, \cdots, x_{m}, t, u\right)$. (Received July 18, 1955.)

774. Jim Douglas, Jr.: The relationship between stability and convergence in numerical solution of partial differential equations.

It is the purpose of this paper to prove that stability in the sense of von Neumann is sufficient to insure convergence in the mean of the solution of a difference analogue of a linear parabolic or hyperbolic differential equation with time independent coefficients to that of the differential equation. No restriction on the number of independent variables nor on the order of the differential equation is involved. The difference equation may be either implicit or explicit in form and may involve the values of its solution on an arbitrary number of time levels. (Received July 18, 1955.)

775. Jim Douglas, Jr. and T. M. Gallie, Jr. (p): Numerical integra- 


\section{tion of a parabolic differential equation subject to a moving boundary condition.}

Physical problems such as melting of ice, recrystallization of metals, evaporation of droplets and invasion processes of reservoir engineering are governed by the heat flow (or other parabolic) equation and a condition on a moving boundary. Locating the boundary and determining the solution of the equation is known as Stefan's problem. A simple form of Stefan's problem is (I): (a) $u_{x x}=u_{t}, 0<x<x(t)$; (b) $u_{x}(0, t)=-1$, $t>0$; (c) $u(x(t), t)=0, t \geqq 0$; (d) $t=x(t)+\int_{0}^{x(t)} u(x, t) d x, t>0$; (e) $x(0)=0$. Existence and uniqueness of the solution of this nonlinear system has been proved recently by several authors, but evaluation of the solution is not yet in a definitive state. An analogous system of difference equations is (II): (a) $\Delta_{x}^{2} u_{i, n+1}=\left(u_{i, n+1}-u_{i n}\right) / k_{n}, i=1, \cdots, n$; (b) $\left(u_{1, n+1}-u_{0, n+1}\right) / h=-1$; (c) $u_{n+1, n+1}=0$; (d) $k_{n}=\left(n+1+\sum_{i=1}^{n} u_{i, n+1}\right) h-\sum_{i=0}^{n-1} k_{i}$. In this paper a method for solving the nonlinear system (II) by iteration on the time step $k_{n}$ is proved to converge. It is then proved that the solution and boundary of (II) converge to those of (I) as $h \rightarrow 0$. Stability with respect to small errors is proved. The method of proof depends on a maximum principle for parabolic difference equations which is of interest in itself. (Received July 18, 1955.)

776. A. C. Downing, Jr.: A finite difference net applicable to multiregion problems. Preliminary report.

In treating multi-region problems by finite difference methods, one of the most awkward questions in setting up a difference scheme which approximates the differential problem is the handling of interior corner points. With lattice systems customarily used, this problem becomes increasingly difficult as the number of space dimensions increases. A very simple grid scheme which overcomes the difficulties associated with corner points is presented in this paper. This grid has the property that all interfaces and boundaries are located midway between adjacent planes of the lattice of the grid points. As an application, in order to demonstrate the simplicity and the usefulness of this grid scheme, the multi-region diffusion problem in $n$ dimensions is considered. In particular, it is shown that all the eigenvalues of the finite difference problem are real under conditions which correspond to the most general conditions, known to the author, which are sufficient to prove the realness of the eigenvalues of the corresponding differential problem. (Received July 18,1955.)

777. G. E. Forsythe: Asymptotic lower bounds for all eigenvalues of a convex membrane.

Let $R$ be a convex plane region bounded by a piecewise analytic curve $C$ with strictly convex corners. Let the eigenvalues $0<\lambda^{(1)}<\lambda^{(2)} \leqq \lambda^{(3)} \leqq \cdots$ and eigenfunctions $u^{(k)}$ be such that $\Delta u^{(k)} \equiv u_{x x}^{(k)}+u_{y y}^{(k)}=-\lambda^{(k)} u^{(k)}$ in $R$ with $u^{(k)}=0$ on $C$. (Subscripts denote derivatives.) Let a self-adjoint finite difference approximation to the above problem be introduced as in G. E. Forsythe, Pacific Journal of Mathematics vol. 5 (1955). Let $0<\lambda_{h}^{(1)}<\lambda_{h}^{(2)} \leqq \lambda_{h}^{(3)} \leqq \cdots$ be its eigenvalues. Lemma: For any $k=1,2, \cdots$ as $h \rightarrow 0$ one has $\lambda_{h}^{(k)} / \lambda^{(k)} \leqq 1-A_{k} h^{2}+o\left(h^{2}\right)$, where $A_{k}>0$. Estimates for $A_{k}$ are given in terms of integrals of $u_{x x}^{(i)} u_{x x}^{(i)}+u_{y y}^{(i)} u_{y y}^{(j)}$ over $R$. Theorem: With the same hypotheses, for each $k=1,2, \cdots$ there exists $h_{0}=h_{0}(k)$ such that $\lambda_{h}^{(k)}<\lambda^{(k)}$ for all $h \leqq h_{0}$. The lemma is proved by finding the stationary values of the Rayleigh quotient for the difference problem over the subspace spanned by the net functions induced by $u^{(1)}, \cdots, u^{(k)}$. (Received July 13,1955 .) 


\section{H. M. Gurk: Five person extreme games.}

An $n$-person game $G$ is extreme provided there do not exist games $G_{1}$ and $G_{2}$ such that $v(S)=a v_{1}(S)+(1-a) v_{2}(S)$ for all $S \subset(1, \cdots, n)$ where $0<a<1$ and where $v, v_{1}$, and $v_{2}$ are the characteristic functions of the three games. von Neumann and Morgenstern have pointed out that all $n$-person simple games are extreme (Theory of games and economic behavior, Princeton, 1947, Chap. 10), but the converse is true only for $n \leqq 4$. Theorem 1: $G$ is an extreme, constant-sum 5-person game if and only if $v(S)$ $\neq 0,1$ for $S \subset(1, \cdots, 5)$ implies that there exists a chain of coalitions $T_{1}, \cdots, T_{2 r}$ such that (i) $T_{1}=T_{2 r}=S$, (ii) $T_{j} \cdot T_{j+1}=\phi$, and (iii) $v\left(T_{j}\right)+v\left(T_{j+1}\right)=1$ for $j=1, \cdots$, $2 r-1$. From this theorem, there are 14 different types of games (excluding permutations of players), 8 of which are nonsimple. Counting permutations there are 294 5-person extreme constant-sum games altogether as compared with 84 -person ones. For a 5-person game $G$, let $W^{m}=\left\{S \subset(1, \cdots, 5) \mid v(S)=1\right.$ and $v(T)<1$ for $\left.T_{p} \subset S\right\}$, and let $W_{I}^{m}=\left\{S \in W^{m} \mid v(S-T)<1-v(T)\right.$ for $T \neq \phi, T_{p} \subset S$. Theorem 2: Let $G$ be a 5-person constant sum extreme game. Let $V_{S}=\{\alpha \mid \alpha$ is an imputation such that $\sum_{S} \alpha_{i}=1$ and $\sum_{T} \alpha_{i} \geqq v(T)$ for $\left.T \subset S\right\}$ for $S \in W^{m}$. Then (1) if $S \in W_{I}^{m}, V_{S}$ is a solution of $G$, and (2) if $W_{I}^{m}=\phi$, then $V=\bigcup_{S \in W^{m}} V_{S}$ is a solution of $G$. (Received June 17, 1955.)

779t. P. C. Hammer, O. J. Marlowe, A. H. Stroud: Numerical integration over simplexes and cones.

Hammer and Wymore have recently made a first step in the direction of a systematic exploration of numerical integration in $n$-dimensional spaces for $n \geqq 2$. They have laid down the general principles of using affine transformations and cartesian products to extend the applicability of every specific formula developed. Specific formulas and methods they have given for numerical integrations over certain types of symmetrical regions. In this paper we develop methods of numerical integration over simplexes and cones and give specific numerical formulas for triangles, tetrahedra, and solid circular cones. The specific theory permits determination of formulas for $n$-simplexes which are exact for polynomials of at most $k$ th degree in $n$ variables. (Received July 5, 1955.)

780. T. W. Hildebrandt: Self-adjointness in one-group, multiregion diffusion problems. Preliminary report.

Consider the differential equation $\nabla^{2} u_{i}+D_{i} u_{i}=\lambda \theta_{i} u_{i}$ holding in each of the $N$ connected subregions $S_{i}$ of the connected region $S ; D_{i}$ and $\theta_{i} \geqq 0$ are constant in each $S_{i}$. On the interfaces $\Gamma_{i j}$ between two adjacent regions, $u_{i}=\alpha_{i j} u_{j}+\beta_{i j} \partial u_{j} / \partial n, \partial u_{i} / \partial n$ $=\gamma_{i j} u_{j}+\delta_{i j} \partial u_{j} / \partial n ; \Delta_{i j}=\left(\alpha_{i j} \delta_{i j}-\beta_{i j} \gamma_{i j}\right) \neq 0$. On the outer boundaries $\Gamma_{i 0}$, similar conditions hold, with $\Delta_{i 0}=0$. Then a sufficient condition that the problem be self-adjoint in the sense that all eigenvalues are real, and eigenfunctions corresponding to distinct eigenvalues are orthogonal, is that $\Delta_{i j}>0$ and $\Delta_{i j} \Delta_{j h} \Delta_{h i}=1$, for all $0<i, j, h \leqq N$. (Received July 14, 1955.)

781t. J. R. Isbell: Absolute games.

A cooperative theory is proposed for general games with continuous but not linear transfer of utility. (See von Neumann and Morgenstern, Theory of games and economic behavior; and several papers of Nash. The former theory assumes linear transfer and the latter is incomplete. The present theory contradicts both.) The NeumannMorgenstern utility axioms are extended to a categorical system in which the utility 
space of a person is a line segment. By normalizing to the segment $[0,1)$ one has interpersonally comparable absolute utility. (von Neumann and Morgenstern refuse to use interpersonal comparison; Nash postulates and exploits its nonexistence.) Solutions of general two-player games are obtained as in Raiffa, Arbitration schemes for generalized two-person games, Annals of Mathematics Studies, no. 28, using the unique arbitration scheme which preserves ratios of base utilities. Then the $n$-person game is treated in terms of its two-player quotient games (as in Neumann-Morgenstern. A player is a coalition of persons). A family of examples shows that a dummy player possessing capital reserves can affect the outcome of a game, and explores the variation of the effect with the utility exchange rate. (Received June 29, 1955.)

\section{J. R. Isbell: A linear value estimate for weapons systems.}

A pair of weapons systems, friendly and enemy, is here described by a pair of matrices. For $m$ friendly weapon types and $n$ enemy types there are an $m$ by $n$ matrix $A$ and an $n$ by $m$ matrix $B$; the typical entry $a_{i j}$ measures the firepower of one $i$-type friendly unit against $j$-type enemy units. A possible interpretation is that $a_{i j}$ is the number of $j$-type enemies which one $i$-type friendly unit can expect to destroy in one hour under certain standard conditions. The entries $b_{i j}$ of $B$ similarly measure enemy firepower (or friendly vulnerability). The proposed value is to satisfy $\lambda x_{i}=\max a_{i j} y_{i}$, $\lambda y_{1}=\max b_{j i} x_{i}$, with some proportionality factor $\lambda$. Thus the values are to be proportional to the maximum possible destructiveness of the units in terms of these same values. Provided $A$ and $B$ are non-negative and neither has a vanishing row, there exist non-negative nonzero values. If all $a_{i j}$ and $b_{j i}$ are positive then so are all $x_{i}$ and $y_{i} ;$ and $\lambda$ is unique. Except for a nondense set of (non-negative) matrices, the values are unique up to a scalar multiple. The values fail to be essentially unique, for strictly positive matrices, if and only if there exists a set of values such that the tactic of maximizing destruction necessarily splits the battle into two unconnected parts. (Received July 14,1955 .)

\section{Joachim Kaiser, Jr.: The effect of bandpass limitations on analogue computer solutions.}

Simple systems of differential equations often solved by the use of electronic analogue computers can be described by expressions of the form $\ddot{y}+2 \zeta \omega \dot{y}+\omega^{2} y=f(t)$. The bandpass characteristics of the operational amplifiers and integrators used to solve these systems normally raise the order of the differential equations giving rise to undesired exponential terms in the solution. The damping ratio $\zeta$ is affected by these exponential terms and a set of curves is derived showing damping ratio errors vs frequency $\omega$. Computer users can consult these curves and obtain an estimate of the accuracies to be expected in the solutions of many problems, particularly in the missile flight simulation field. When electromechanical servos are used in the simulation of missile flight systems the errors introduced by the bandpass of the servos can seriously restrict the use of the computing equipment. Curves are derived showing the bandpass requirements of the computing servos as a function of frequencies and damping ratios encountered in the missile system for computing servos with simple second order transfer functions. (Received July 11, 1955.)

784. L. R. Langdon: Approximation of functions for machine computation. Preliminary report.

Polynomial and Pade function methods for the economical approximation of the arc tangent, arcsin, log, exponential, $x^{n}$ ( $n$ not an integer) and the gas tables are dis- 
cussed. Means of minimizing the error due to the truncation of series and the truncation of coefficients are outlined. (Received July 11, 1955.)

\section{Imanuel Marx: On the "principal axis transformation" for complex quadratic forms.}

To the quadratic form $Q=\sum_{j, k=1}^{n} \alpha_{j k} \xi_{j} \xi_{k}, \alpha_{j k}=\alpha_{k j}$ complex numbers, $\xi_{j}$ complex variables, corresponds the complex symmetric transformation $A$ with matrix $\left(\alpha_{j k}\right)$. An $n$-dimensional vector space $S$ over the complex numbers, non-isotropic relative to a complex symmetric scalar product, is mapped into itself by $A$, and $S$ is a direct sum of mutually orthogonal, indecomposable, non-isotropic subspaces invariant relative to $A$. Hence there exists a complex orthogonal matrix $\left(\tau_{\jmath k}\right),\left(\tau_{l k}\right)\left(\tau_{k_{l}}\right)=\left(\delta_{j k}\right)$, such that $\left(\tau_{j k}\right)\left(\alpha_{3 k}\right)\left(\tau_{j k}\right)^{-1}$ is a simple canonical form. Correspondingly, $Q$ is transformed by $\eta_{g}=\sum_{h=1}^{n} \tau_{g h} \xi_{h}, \sum_{g=1}^{n} \eta_{g}^{2}=\sum_{h=1}^{n} \xi_{h}^{2}$, to a "principal axis" form, which displays, in addition to the characteristic values, parameters independent of the characteristic equation. (This work continues a program initiated by C. L. Dolph and described in Comm. Pure Appl. Math. vol. 7 (1954) p. 622.) (Received July 18, 1955.)

786. M. E. Muller: On discrete operators connected with the Dirichlet problem.

An approach is suggested to determine optimum operator approximations and admissible networks for finite difference techniques. A detailed study is made for the Dirichlet problem. The approach looks promising for the higher dimensional problems which will depend for their solution on the use of high speed electronic computing equipment. For the two-dimensional Dirichlet problem this approach adds further justification for the networks already in use. Contrary to popular belief, it is shown that when the dimensionality of the problem is higher than two, it is not always possible to obtain better finite difference operators by increasing the number of neighboring points that the operators will utilize. Attention is then given to showing that Monte Carlo estimates for the $\mathrm{N}$-dimensional Dirichlet problem can be obtained by using random walks on the closed neighbor simplex networks that are introduced. The results of the paper may also be applied to relaxation methods. (Received June 22, 1955.)

\section{G. Y. Rainich: Determination of electric and magnetic vectors in} terms of stresses in general relativity theory.

It has been proved before that the electromagnetic stress-energy tensor $S$ when given only at a point does not determine uniquely the field tensor $F$ (whose components interpreted three-dimensionally are the components of the electric and magnetic force vectors); more precisely, there is a one-parameter set of $F$ 's that give the same $S$. It has also been shown, however, that when $S$ is given in a portion of space-time containing discrete charged particles, $F$ can be calculated. The case of continuously distributed charged matter (represented by a tensor $M$ ) has not been considered so far. In the present paper expressions are derived which determine $F$ uniquely in terms of $S$, its divergence, and $M$. The importance of this lies in the fact that it permits one to arrive at the conclusion: the curvature of space-time determines both matter and electromagnetic field, since it has been shown before that the curvature tensor (in the nonsingular case) can be split uniquely into $S$ and $M$. The paper also contains a new proof of this last proposition. (Received July 18, 1955.) 
788. D. E. Richmond: On the existence of limit cycles.

The existence of a limit cycle for $x^{\prime \prime}+f\left(x, x^{\prime}\right) x^{\prime}+g(x)=0\left({ }^{\prime}=d / d t\right)$ is proved by applying Bendixson's theorem to an unusually simple ring-shaped region in the $x x^{\prime}$ plane, under the following conditions on the continuous functions $f$ and $g$ : (I) $f\left(x, x^{\prime}\right)$ $>0$ if $|x|>a, f(0,0)<0, f\left(x, x^{\prime}\right) \geqq-k$ if $|x|<a$; (II) $g(x)$ increases monotonically and $g(0)=0$; (III) The existence of a positive function $\phi$ such that $f(x, \phi(x))$ $\geqq-g(x) / \phi(x)-\phi^{\prime}(x)$ if $x \leqq-b<-a$. Each of these conditions may be relaxed. Thus in (I), $f\left(x, x^{\prime}\right)$ may be negative ( $\geqq-m$ ) for $x>a$ with properly chosen $m$ (in some cases, arbitrarily large). (Received July 15,1955 .)

789. A. E. Roach: The effect of particle-size distribution on wear rate.

An energy-balance analysis of wear rate requires the introduction of a term containing the particle-size distribution. This paper shows that the term is relatively independent of the size-distribution function and can be expressed as a simple function of the mean particle size provided the mean is properly defined. (Received July 11, 1955.)

790. Seymour Sherman: On Segal's postulates for general quantum mechanics.

In [Postulates for general quantum mechanics, Ann. of Math. vol. 48 (1947) pp. 930948] I. E. Segal has presented "a set of postulates for a physical system" and deduced from these "the main general features of the quantum theory of stationary states." An important way in which he differed from his precursors, P. Jordan, J. von Neumann, and E. P. Wigner, was by not requiring a distributive postulate, which according to them was a postulate lacking physical meaning. The only models he presented for his system were, in essence, uniformly closed, self-adjoint algebras of bounded operators on real or complex hilbert space. Natural questions that arise are (1) Are there any other models for Segal systems? (2) Are Segal systems necessarily distributive? (3) Does an analogue to the Stone-Weierstrass Theorem hold for Segal systems? (4) Are lattice-ordered Segal systems necessarily commutative? These questions are answered by presenting a class of systems of observables which satisfy Segal's postulates yet do not form algebras since the distributive law fails to hold. For these examples at least one analogue of the Stone-Weierstrass Theorem fails to hold. An example is given of a system of observables which is a lattice in its natural order but is not commutative. With the aid of a remark of Albert it is shown that $M_{3}^{8}$, the set of $3 \times 3$ matrices whose elements are Cayley numbers, constitutes a Segal system. It is shown that Postulate II, 4 of Segal's paper is redundant. (Received May 25, 1955.)

791. Patricia J. Wells: The integral equation for the prolate spheroidal antenna.

The Albert and Synge integral equation [Quarterly of Applied Mathematics vol. 6 (1948)], satisfied by the current on a surface of revolution, is solved for the case of the prolate spheroid. The solution is found by means of the expansion of an angularly symmetric dipole field in terms of the prolate spheroidal wave functions of order one. Comparison of this expansion with a known expansion in terms of functions of order zero yields recursion relations between the spheroidal wave functions of orders one and zero. (Received July 18, 1955.) 


\section{GEOMETRY}

\section{2t. Louis Auslander: On the prolongation of exterior differential equations.}

Let $\Sigma$ be a system of exterior differential equations in a coordinate neighborhood $U$ and let $x^{i}, i=1, \cdots, g$, be independent variables and $z^{\alpha}, \alpha=1, \cdots, n-g$, be dependent variables. Assume that over the point $u \in U$ there exist involutive prolongations of all orders. Then a set of prolongations, one of each order, uniquely determines a set of formal power series $z^{\alpha}=a_{i}^{\alpha}+\sum a_{\imath}^{\alpha} x^{i}+(1 / 2 !) \sum a_{i j}^{\alpha} x^{i} x^{j}+\cdots$ with the property that if each of the above series converge the surface thus determined will be an involutive integral surface for $\Sigma$. (Received June 17, 1955.)

793. K. D. Fryer (p) and Israel Halperin: On the coordinatization theorem of J. von Neumann.

A precise statement of the von Neumann theorem is as follows. Let $L$ be a complemented modular lattice possessing a homogeneous basis $a_{1}, \cdots, a_{n}, n \geqq 4$, and let $L_{12}$ be the set of all inverses of $a_{2}$ with respect to $a_{1}+a_{2}$. Then (i): addition and multiplication can be defined for the elements of $L_{12}$ is such a way that $L_{12}$ becomes a regular ring $\Re$ and the sublattice $L\left(a_{1}\right)$, consisting of all $x \leqq a_{1}$, is isomorphic to the lattice of all left principal ideals of $\Re$, and (ii): $L$ is lattice-isomorphic to (coordinatized by) the lattice of all left principal ideals of $\Re_{n}$. Here $\Re_{n}$ consists of all $n \times n$ matrices with elements in $\Re$ and, as shown by von Neumann, is regular along with $\Re$. In the present paper, a direct proof is given for (ii)' (equivalent to (ii)) $L$ is latticeisomorphic to (coordinatized by) the lattice of $\Re$-left modules of finite span in the space $V$ of vectors $\left(\alpha^{1}, \cdots, \alpha^{n}\right)$, all $\alpha^{i}$ in $\Re$. (Received July 1, 1955.)

\section{David Gale: On convex polyhedra.}

Let $V$ be the set of vertices of a convex polyhedron $P$. The vertices belonging to a subset $S$ of $V$ are called neighbors if $S$ is the intersection of $V$ with a supporting hyperplane to $P$. If every $m$ vertices of $V$ are neighbors then $P$ is called $m$-neighborly. Suppose $P$ is $k$-dimensional and $m$-neighborly, $m \leqq k$. If $k<2 m$, one easily shows that $P$ must be a $k$-simplex. It is shown, on the other hand, that if $k \geqq 2 m$, then $P$ may have any number of vertices. As a special case this shows that there exist polyhedra in 4 -space with arbitrarily many vertices every two of which are connected by an edge. (Received July 7, 1955.)

\section{Michael Goldberg: Central tesselations.}

Regular tesselations, discussed in many books and papers, are periodic arrangements of congruent regions covering the infinite plane. Heesch [Nachrichten von der Gesellschaft der Wissenschaften zu Göttingen N.S. vol. 1 (1935) pp. 115-117] and Voderberg [Jber. Deutschen Math. Verein. vol. 46 (1936) pp. 229-231; vol. 47 (1937) pp. 159-160] gave examples of tesselations which are not regular. The present paper presents several types of nonregular tesselations which are characterized by the possession of a unique center. They include sector, spiral, concentric polygon, vortex, dodecagonal and parallel core tesselations. Modifications of regular honeycombs to central honeycombs are given as examples of extension to three dimensions. (Received July 11, 1955.)

796t. S. I. Goldberg: Note on semi-symmetric connections in Hermitian manifolds. 
Consider a complex-analytic manifold $M^{n}$ of $n$ complex dimensions endowed with a (general) Hermitian metric. The author defines formally (as analogues of the Kaehler case) manifolds of "constant" curvature and "constant" holomorphic curvature. More generally, Einstein spaces are introduced with the various contractions of the curvature tensor. If the contracted torsion tensor vanishes $\left(S_{i i}^{i}=0\right)$, the manifold is Kaehlerian. If the scalar curvature is not constant $\left(S_{j i}^{i} \neq 0\right)$, it is shown that the connection is semi-symmetric and that an Einstein space can be transformed conformally into a Kaehler manifold. Moreover, if $M^{n}$ is compact and $\Delta(\log |\lambda|) \geqq 0(\leqq 0)$ for some scalar field $\lambda, M^{n}$ is torsion free and the conformal map is a similitude. It follows that a compact manifold of "constant" (nonvanishing) holomorphic curvature is torsion free and is, in fact, a Hodge manifold. (Received July 21, 1955.)

797. C. C. Hsiung: Some characterizations of closed umbilical hypersurfaces in Riemannian space of constant curvature.

Let $V^{n}$ be an orientable hypersurface, with a closed boundary $V^{n-1}$ of dimension $n-1(n \geqq 2)$, differentiably of class $C^{3}$ imbedded in an $(n+1)$-dimensional Riemannian space $R^{n+1}$ of constant curvature; $\kappa_{1}, \cdots, \kappa_{n}$ the $n$ principal curvatures at a point $P$ of $V^{n}$; and $M_{i}$ the $i$ th mean curvature of $V^{n}$ at $P$ defined by $C_{n, i} M_{i}=\sum \kappa_{1} \kappa_{2} \cdots \kappa_{i}$ $(i=1, \cdots, n)$, where the expression on the right side is the $i$ th elementary symmetric function of $\kappa_{1}, \cdots, \kappa_{n}$, and $C_{n, i}$ denotes the number of combinations of $n$ different things taken $i$ at a time. Let $d A$ be the area element of $V^{n}$ at $P$, and $p$ the scalar product of the unit normal vector of $V^{n}$ at $P$ and the position vector of $P$ with respect to any orthogonal frame in the space $R^{n+1}$. The purpose of this paper is first to show that the integral $\int_{V^{n}}\left(M_{n-1}+M_{n} p\right) d A$ can be expressed as an integral over the boundary $V^{n-1}$ and then from this integral formula and another one obtained by the author [Bull. Amer. Math. Soc. Abstract 61-2-331] to deduce some simple conditions for a closed $V^{n}$ to be umbilical. For a Euclidean space $R^{n+1}$ these results were obtained in a previous paper by the author [Math. Scand. vol. 2 (1954) pp. 286-294]. (Received July 11, 1955.)

798. W. R. Hutcherson: A note on nonequivalent cyclic homographies.

J. Dessart [Sur les surfaces representant l'involution engendré par un homography de cinque du plan, Memoires de la Société Royale des Sciences de Liège no. 17 (1931) pp. 1-23] and other writers have used the homography $x_{1}^{\prime}: x_{2}^{\prime}: x_{3}^{\prime}=x_{1}: E x_{2}: E^{\alpha} x_{3}$ where $E^{p}=1$ ( $p$ is prime) and $\alpha$ (positive integer) $\leqq p$. Of the $p^{2}$ homographies, there are $p$ sets, each containing $p-1$ equivalent (each generates the same $p$ set of points) homographies. For example, the two equivalent homographies $\left(x_{1}, E^{p-1} x_{2}, E x_{3}\right)$ and $\left(x_{1}, E x_{2}, E^{p-1} x_{3}\right)$ are found in only one of the $p$ sets. If $x_{2}$ and $x_{3}$ are interchanged and one excludes those collineations which relate to perfect points [W. R. Hutcherson and J. C. Morelock, Concerning a pattern for perfect points, Bull. Amer. Math. Soc. Abstract 60-6-554] it is discovered that there are exactly $(p-1) / 2$ distinct nonequivalent sets of homographies for each prime number $p$. (Received June 9, 1955.)

\section{9t. Harold W. Kuhn: On certain convex polyhedra.}

Let $T_{n}$ be the set of tours (i.e., cyclic arrangements of $1, \cdots, n$ ) represented as $n$ by $n$ permutation matrices $t=\left(t_{i j}\right)$. Let $C_{n}$ denote the convex hull of $T_{n}$ in $n^{2}$ dimensional Euclidean space. The polyhedron $C_{n}$ spans the $\left(n^{2}-3 n+1\right)$-dimensional linear variety of all $x=\left(x_{i j}\right)$ with $x_{i i}=0$ and $\sum_{j} x_{i j}=\sum_{i} x_{i j}=1$ for all $i$ and $j$. Every nonzero matrix $b=\left(b_{i j}\right)$ defines a half-space $\sum b_{i j} x_{i j} \geqq \beta$ supporting $C_{n}$ by the rule: $\beta=$ minimum of $\sum b_{i j} t_{i j}$ for $t=\left(t_{i j}\right) \in T_{n}$. All faces of $C_{n}$ (i.e., $\left(n^{2}-3 n\right)$-dimensional 
intersections of $C_{n}$ with supporting hyperplanes) can be obtained from non-negative, integral $b$. For $n<5$, matrices of zeros and ones suffice. For $n=5$, the following two classes: $x_{12}+x_{13}+x_{21}+x_{25}+x_{31}+x_{34}+x_{42}+x_{53} \geqq 1$ (60 distinct faces with renumbering of $1, \cdots, 5)$ and $x_{13}+2 x_{14}+x_{15}+x_{23}+x_{25}+x_{31}+x_{32}+x_{41}+x_{42}+x_{45}+2 x_{61} \geqq 2 \quad(120$ faces) together with the 210 faces given by Heller [Bull. Amer. Math. Soc. Abstract 59-6-664] describe $C_{5}$ in an irredundant manner. (Received July 13, 1955.)

\section{0t. B. Z. Linfield: Integral and matric geometry.}

With functions as coordinates and integrals as distances, any orbit, any velocity or acceleration of a particle in its orbit, any surface, any space, any direction in a space, is determined by one scalar equation. Each set of points, be it a curve, a surface, a space, or a point like a centroid, is characterized by one scalar equation $f=f(z, u)$, where each value of $u$ determines one point in the set, and where the number of components in $u$ determines the dimension of this $f$-space $f(z, u)$. The square of the distance between two points $f_{1}=f\left(z, u_{1}\right)$ and $f_{2}=f\left(z, u_{2}\right)$ is $\int\left(f_{1}-f_{2}\right)^{2} d z$. The limits of integration are here from $-\pi$ to $\pi$. Every covariant $X$ in $f(z, u)$ is a matric function, which satisfies a polynomial equation with invariant coefficients; and is linearly dependent on basic covariants, which satisfy polynomial equations with numerical coefficients. Every invariant $F$ in $f(z, u)$ is a scalar function whose value at every point is independent of the choice of $u$-coordinates. For a Euclidean plane or space $f=f(z, u)$ a certain covariant $Z(f)=0$. (Received July 5, 1955.)

\section{1t. B. Z. Linfield: Covariant functions of invariants in f-space.}

Every invariant $F$ in $f(z, u)$ has a covariant $Z(F)$ such that the leading coefficient the equation $Z(F)$ satisfies is the Laplacian $L(F)$. It has a covariant $Y(F)$ which satisfies $Y^{2}(F)=Y(F)=Y(G)$, for any invariant $G=G(F)$. Any two invariants $(F, G)$ have an invariant $T(F, G)=T(G, F)$, and two conjugate covariants $Z(F, G), Z(G, F)$ which satisfy the same quadratic equation $X^{2}=T(F, G) X$. When $G$ depends functionally on $F$, its covariant $Z(G)$ is linearly dependent on $Z(F)$ and $Y(F)$, the covariants $Z(F, G), Z(G, F) Y(G)$ are linearly dependent on $Y(F)$, the invariant $L(G)$ is linearly dependent on $L(F)$ and $T(F, F)=k^{2}$, and $T(G, G)=k^{2}(d G / d F)^{2}, Z(F, F)$ $=k^{2} Y(F)$. For any surface $f(z, u), \operatorname{dim} u=2$, in 3-dimensional space; and any $n$-dimensional surface $f(z, u), \operatorname{dim} u=n$, in $(n+1)$-dimensional space, the covariant $Z(f)$ is factorable, i.e. $Z(f)=P(u) q(z, u)$, where covariant $P(u)$ depends functionally on $u$ only, and invariant $q(z, u)$ is the direction of the normal to $f(z, u)$ and satisfies $\int q^{2} d z$ $=1=\int f(z, u) d z$. For covariant $P(u)$ there exist two covariant functions $C(V, X)$, $D(X, U)$, of any covariant $X$, such that $C(V, P)=D(P, U)=0$. When $\operatorname{dim} u=2$, then $Z^{2}(f)=L(f) Z(f)-q^{2}|P| I$. (Received July 5, 1955.)

\section{2t. B. Z. Linfield: Covariant functions of covariants in $f$-space.}

Any covariant vector $U$, any contravariant vector $V$, and any covariant $X$, have three covariants $\theta(X)=\theta(V, X), \theta=A, C, E$; and two covariants $\theta(X)=\theta(X, U)$, $\theta=B, D$. These five covariants $\theta(X)$ satisfy like $X$ polynomial equations with invariant coefficients, are linear in $X, U, V: \theta(X+Y)=\theta(X)+\theta(Y)$, etc., and $\theta(X Y)$ satisfy simple functional equations. For any surface $f(z, u)$, where $Z(f)=P(u) q(z, u)$, the covariant $Z(q)$ is linearly dependent on orthonormal directions $q, w_{1}, w_{2}, \cdots$ with coefficients $-P^{2}$ and $\theta(P)$, i.e. $Z(q)=-q P^{2}+\sum_{1} w_{1} B\left(P, U_{1}\right) / k_{1}$, where $\int w_{1} w_{2} d z=0$ $=\int q(z, u) d z$, etc. For any sphere, or circular cylinder, we get $Z(q)=-q P^{2}$. For the sphere, the determinant $|Z(q)|=(q|P|)^{2} \neq 0$. There are similar covariant equations to characterize other surfaces. Practically every scalar, vector, or matric equation in 
function coordinates takes the place of many scalar, vector, or matric equations not in functions coordinates. They are therefore more economical than tensor equations, and much easier to use and understand. (Received July 5, 1955.)

803t. Albert Nijenhuis: Scalar fields whose second covariant derivatives are equal to a given Riemannian metric tensor.

The problem, proposed by E. T. Parker (Bull. Amer. Math. Soc. Research Problem 61-3-19), is when there exists in a Riemannian space (not necessarily positive definite) a scalar function $s$ such that $\nabla_{b} \nabla_{a} s=g_{b a}$. Putting $v^{a}=g^{a b} \partial_{b} s$ one finds that for each $s$ there is a vector field $v^{a}$ satisfying $\nabla_{b} v^{a}-\delta_{b}^{a}=0$, i.e. $v^{a}$ is invariant under the Cartan connection of "rollings" of the tangent space. Every vector $v \in T_{p}$ (tangent space at $p$ ) invariant under the corresponding holonomy group $H(M, p)$ gives rise to such a vector field. Once $v^{a}$ is formed, $s$ is solved from the equations $\partial_{a} s=g_{a b} v^{b}$, which are then (locally) completely integrable. For the local existence of $s$ one only needs vectors $v \in T_{p}$ invariant under the local holonomy group $H^{*}(p)$. If $M$ is compact or complete no vector fields $v^{c}$, and therefore no scalar fields $s$ of the proper kind, exist, except when $M$ is Euclidean space with a Euclidean metric. General reference: A. Nijenhuis, On the holonomy groups of linear connections [Proc. K. Akad. Wetensch. A vol. 56 (1953) pp. 233-249, vol. 57 (1954) pp. 17-25]. (Received July 1, 1955.)

804t. R. Z. Norman: On the convex polyhedra of the symmetric traveling salesman problem.

In the symmetric traveling salesman problem, a tour (cyclic arrangement of $1, \cdots, n)$ need not be distinguished from its opposite (the same arrangement in opposite order). Both tours can be represented by a triangular array of elements $t=\left(t_{i j}\right)$ of an $n \times n$ matrix for which $i<j$, in which $t_{i j}$ is 0 or 1 and in which for all $j$, $\sum_{i<j} t_{i j}+\sum_{i>i} t_{j i}=2$. The tours are thus points in an $\left(\left(n^{2}-3 n\right) / 2\right)$-dimensional manifold of an $\left(\left(n^{2}-n\right) / 2\right)$-dimensional Euclidean space. Let $C_{n}$ denote the convex hull of these points. A face of $C_{n}$, an intersection of $C_{n}$ with a supporting hyperplane, can be described by means of the corresponding supporting half space. For $n=5$, as is already known, there are two classes of these face-defining supporting half-spaces, I : $x_{i j} \geqq 0$ and II : $x_{i j} \leqq 1$, each having 10 half-spaces. For $n=6$ there are 15 half-spaces in each of class I and class II, 10 in class III: $x_{i j}+x_{i k}+x_{j k} \leqq 2$, and 60 in class IV: $x_{i j}+x_{j k}+x_{i k}+x_{i l}+x_{i m}+x_{k n} \leqq 4$. For $n=7$ there are 21 in each of the first two classes, 35 in Class III, 840 in class IV, and 1260 in class V: $x_{i j}+x_{j k}+x_{i k}+x_{k m}+x_{k n}+x_{m n}$ $+x_{m p}+x_{n q} \leqq 5$. These supporting half-spaces were found independently by Dantzig and Johnson. The proof that the faces corresponding to these sets of half-spaces give an irredundant description of the convex sets $C_{n}$ is in part the same as Kuhn's proof of the corresponding statement for the convex of all tours (see Abstract 799), and follows in part from a graph-theoretic argument. (Received July 18, 1955.)

\section{5t. T. K. Pan: On families of curves in a surface.}

Associate a surface $S$ in Euclidean space with a congruence of unit vectors $\lambda$ which make a constant angle with the surface normals. A curve in $S$ is a line of $\lambda$-projectile if along it the component vectors in $S$ of the derived vectors of the $\lambda$-congruence are tangent. A curve in $S$ is a line of $\lambda$-orthoprojectile if along it the component vectors in $S$ of the derived vectors of the $\lambda$-congruence are orthogonal. A line of $\lambda$-projectile coincides with a line of curvature of $S$ if the component vectors in $S$ of the $\lambda$-congruence are either orthogonal to or parallel along the line of $\lambda$-projectile. A line of $\lambda$-orthoprojectile coincides with an asymptotic line of $S$ if the component vectors in $S$ of the 
$\lambda$-congruence are either tangent to or parallel along the line of $\lambda$-orthoprojectile. A plane geodesic or a geodesic line of curvature is a line of projectile, a geodesic asymptotic line, or a straight line in $S$ is a line of orthoprojectile. (Received July 18, 1955.)

806. C. M. Petty and Daniel Waterman (p): An extremal theorem for $N$-simplexes.

Comparing the sum $R_{0}+R_{1}+R_{2}$ of the distances from the vertices of a triangle to an arbitrary point $P$ with the area $\Delta$ of the triangle, L. Fejes Toth gives the relation $R_{0}+R_{1}+R_{2} \geqq 2 \cdot 3^{1 / 4} \Delta^{1 / 2}$, equality holding if and only if the triangle is equilateral and $P$ is its centroid. This result is generalized to $n$ dimensions. Theorem: If $R_{i}(i=0, \cdots$, $n)$ are the distances from an arbitrary point $P$ to the vertices of a nondegenerate simplex in $E^{n}$ of volume $\Delta$, then $R_{0}+\cdots+R_{n} \geqq(n+1)^{(n-1) / 2 n} n^{1 / 2}(n !)^{1 / n} \Delta^{1 / n}$ where the equality holds if and only if the simplex is regular and $P$ is its centroid. The proof of the theorem is elementary, utilizing a characterization of orthocentric simplexes developed there. The theorem is also formulated as an inequality concerning determinants. (Received July 18, 1955.)

\section{I. M. Singer: Transitive holonomy groups. II.}

Let $C^{m}$ be the manifold of complex projective space of $m$ homogeneous variables, and let $S^{2 m-3}$ be the space of rays through the origin of the tangent space at a point of $C^{m}$. If $m$ is odd, the holonomy group of any affine connection on $C^{m}$ acts transitively on the sphere $S^{2 m-3}$. Similarly let $Q^{m}$ be the manifold of quaternion projective space of $m$ homogeneous variables, with $S^{4 m-1}$ the rays through the origin. If $m$ is not divisible by twenty-four, then the holonomy group of any affine connection acts transitively on $S^{4 m-1}$. These theorems are proved by using the fact that $S^{2 m-1}$ is a bundle over $C^{m}\left[S^{4 m+3}\right.$ is a bundle over $\left.Q^{m}\right]$ and computing the characteristic map of the bundle $p^{-1}(B)$ where $p$ is the projection map of $S^{2 m-1}$ onto $C^{m}\left[S^{4 m-1}\right.$ onto $\left.Q^{m}\right], B$ is the bundle of bases over $C^{m}\left[Q^{m}\right]$, and $p^{-1}(B)$ is the induced bundle. (Received July 18, 1955.)

\section{LOGIC AND FOUNDATIONS}

\section{Kurt Bing: On simplifying propositional formulas.}

A necessary and sufficient condition, not depending on developed normal formulas, is obtained for a given propositional formula to be implied by the irredundant conjunctions of negated and unnegated propositional letters first investigated by Quine (Amer. Math. Monthly vol. 59 (1952) pp. 521-531; Bol. Soc. Mat. Mexicana vol. 10 (1953) pp. 64-70). Among the consequences is an extension of the known conditions under which a disjunction of conjunctions is reduced to simplest normal form by deletion of those conjunctions which contain other conjunctions as parts. (Received July 18, 1955.)

\section{9t. Ben Dushnik: Mixed sums of order types. Preliminary report.}

Let $A$ and $B$ be two simply ordered sets, and let $C$ be the union of the (unordered) sets $A$ and $B$. Define a simple ordering on $C$ in any manner compatible with the sole requirement that the elements of $A$ and of $B$ retain their original orderings. If $\alpha$ and $\beta$ are the order types of $A$ and $B$, and if $\Gamma:\left(\gamma^{\prime}, \gamma^{\prime \prime}, \ldots\right)$ is the set of all possible order types which $C$ may have when ordered as above, then $\Gamma$ will be called the mixed sum of $\alpha$ and $\beta$. Hausdorff's "natural" sums of ordinals is an instance of (an element of) a mixed sum; the present paper indicates possible uses of mixed sums. (Received July 19, 1955.) 


\section{P. C. Hammer: The foundations of general topology.}

Let $M$ be a set and $f$ a function mapping a class $D$ of subsets of $M$ onto a class $R$ of subsets of $M$. A set $X$ in $M$ is $f$-closed if and only if $X \supset Y, Y \in D$ implies $X \supset f Y$. To each $f$ there corresponds a limit function $f_{1}$ defined as a subclass $D_{1}$ of $D$ such that the intersection of $Y$ and $f_{1} Y$ is empty for $Y \in D_{1}$ and such that the class of $f_{1}$-closed sets coincide with the class of $f$-closed sets. With each $f$ there are associated two uniquely determined functions $g$ and $h$ defined for all subsets of $M$. The function $g$ is an inclusion preserving enlargement function and the function $h$ is a closure function. Using these functions a critical analysis of the structure of general topology is made, revealing the necessity of a new definition of limit point and continuity and extending general theorems on connectedness to more general forms of connectedness. (Received July $5,1955$. )

811t. A. C. Sugar: Axiomatic systems for classical and relativistic timeless dynamics. Preliminary report.

One of the most pronouncedly counterintuitive concepts in relativistic mechanics is the notion of time. Intuitively the notion of time stems from our concepts of motion and can be defined in terms of our notions of distance and uniform motion. In order to clarify our concept of time in general, and in the particular circumstance of relativistic dynamics, the author formalizes mechanics in timeless form. Acceleration is expressed in the familiar form in which we take the product of velocity expressed as a point function and its derivative, namely, $v d v / d s$. The second law expressed heuristically becomes then $F=m v d v / d s$. Although the axioms will parallel those of a previous paper (Journal of Rational Mechanics and Analysis, April 1953) by the author and J. C. C. McKinsey, one has the important exception that force and velocity are functions of position instead of time. Clearly there exists a class of realizations of these axioms in which the above function " $v$ " may designate a "generalized" or pseudo velocity in terms of which one may define a pseudo time. Hence dynamics formalized in this fashion embraces new generalizations of classical and relativistic mechanics. (Received July 13,1955 .)

\section{Statistics And Probability}

812t. D. G. Austin: Differentiation properties of Markoff functions where the terminal state is stable.

The author considers a Markoff matrix $\left\{p_{i j}(t)\right\}$ under hypothesis on $\left\{p_{i j}(t)\right\}$ used in the author's note (Proc. Nat. Acad. Sci. U.S.A. vol. 41, pp. 224-226). It is shown that if the terminal state is stable $\left(D p_{j i}(0) \neq-\infty\right)$ then each of the functions $p_{i j}(t)(i=1,2, \cdots)$ has a continuous first derivative satisfying the system of differential equations: $D p_{i j}(t s)=\sum_{k=1}^{\infty} p_{i k}(t) D p_{k j}(s), t \geqq 0, s>0$. (Received July 19, 1955.)

\section{3t. H. A. Bender: Bivariate distribution.}

If one expresses the integral for bivariate distribution in polar form, assuming the origin to be at the mean of the two variates, and supposes $\rho=a \sec \theta$, or $\rho=a \csc \theta$, then this integral simplifies to $\left(e^{-a / 2} / 2 \pi\right) \int_{0}^{t}\left(e^{-a t^{2} / 2} /\left(t^{2}+1\right)\right) d t=\int_{0}^{t} f(a, t) d t=F(a, t)$. The integral $\int_{0}^{t} f(a, t) d t$ can play a role for bivariate distribution very similar to the role played by the probability integral $\int_{0}^{t} \theta(t) d t$. In addition to the above application, one has the following interesting relations: (I) $F(a, t)=2 F(a, 1)-\int_{0}^{a} \theta(t) d t \int_{a}^{a t} \theta(t) d t$ $-F(a t, 1 / t)$. (II) $F(a, \infty)=2 F(a, 1)-\int_{0}^{a} \theta(t) d t \int_{a}^{\infty} \theta(t) d t$. (III) $2 \int_{0}^{\infty} f(a, t) d t=\int_{a}^{\infty} \theta(t) d t$ 
$=1 / 2-\int_{0}^{a} \theta(t) d t$. A table of values for $F(a, t)$ has been computed for $a=1 / 2,1,3 / 2,2$, and $5 / 2$. This table is rather brief since, for example, to four decimal places $F(1,2.8)$ $=F(1, \infty)=0.0793$. With such a table it is possible to find the probability $a_{1} \sigma_{x}<x$ $<a_{2} \sigma_{x}$ and $b_{1} \sigma_{y}<y<b_{2} \sigma_{y}$ for any given value of the correlation between $x$ and $y$ and for certain values of $a$. (Received May 13, 1955.)

814t. J. L. Brenner and G. H. Weiss: On a general form of renewal theory. Preliminary report.

Ordinary renewal theory can be regarded as a study of a one-dimensional unidirection random walk, with steps of varying length. This is generalized to the multidimensional case. In particular the author assumes an $n$-dimensional random walk in which the walker may take a step of varying length in the direction of any axis. A vector of probability densities, $\left[\alpha_{j}(x)\right]$, for the length $x$, of a step in the $j$ th direction is assumed known, as well as a constant irreducible matrix $P\left[=\left(p_{i j}\right)\right]$, of conditional transition probabilities. The element $p_{i j}$ is the probability that a step in the $j$ th direction will be followed by one in the $i$ th direction. A vector integral equation is found to describe this process. A uniqueness and existence theorem is found for this equation. Asymptotic results for the projection along any axis are given, in terms of principal diagonal minors of the matrix $I-P$, where $I$ is the unit matrix. The results are shown to be consistent with certain theorems on determinants with weakly dominant main diagonals. (Received July 20, 1955.)

\section{D. A. Darling ( $\mathrm{p}$ ) and Mark Kac: On occupation times for Markoff processes.}

Let $X_{n}, n=0,1, \cdots$, be a Markoff chain taking on values in an abstract measure space $(\Omega, \mathcal{F})$ with transition probability $P(x, E)=\operatorname{Pr}\left\{X_{k+1} \in E \mid X_{k}=x\right\}, x \in \Omega, E \in \mathcal{F}$. Suppose that $V(x), x \in \Omega$, is real-valued, non-negative and measurable, and that $\sum_{n=0}^{\infty} P^{(n+1)}(x, E) z^{n}=h(z) Q(E)+h_{1}(x, z, E)$ where $P^{(k)}(x, E)$ is the $k$-step transition probability, $h(z) \rightarrow \infty, z \rightarrow 1,0<\int V(x) Q(d x)<\infty,(1 / h(z)) \int V(x) h_{1}(x, z, d x) \rightarrow 0, z \rightarrow 1$. Then the necessary and sufficient condition that there exist constants $a_{n}$ such that $\left(1 / a_{n}\right) \sum_{k=1}^{n} V\left(X_{k}\right)$ has a nondegenerate limiting distribution is that $h(z)$ is $(1-z)^{-\alpha}$ $\cdot L(1 /(1-z)), 0 \leqq \alpha<1$, where $L(x)$ is a slowly varying function. We can then choose $a_{n}=h(1-1 / n)$ and the limiting distribution is necessarily a Mittag-Leffler-Pollard distribution of index $\alpha$. Exactly the same theorems result, mutatis mutandis, for a process $X(t)$ with continuous time. These results give immediately, and generalize, known theorems on duration times, renewal theory, changes of sign, etc. for sums of independent random variables. (Received July 19, 1955.)

816t. Aryeh Dvoretzky, Jack Kiefer, and Jacob Wolfowitz: Asymptotic minimax character of the sample distribution function and of the classical multinomial estimator.

The asymptotic minimax character of the sample distribution function (d.f.) for estimating an unknown d.f. $F$ (in the class $C$ of all continuous univariate d.f.'s, or of all univariate d.f.'s) is proved for a wide variety of weight functions. The weight functions include nondecreasing functions of the normalized maximum deviation $n^{1 / 2} \delta_{n}$ between estimated and true d.f., weight functions obtained as integrals, over the line, of nondecreasing functions of the deviation at individual points on the line, and many other weight functions for which the risk function of the sample d.f. need not even be constant over $C$. For the multinomial estimation problem, the classical estimator is 
shown to be asymptotically minimax for a wide variety of weight functions. Byproducts of the investigation are a general discussion of minimization of symmetric convex or monotone functionals of symmetric random processes, and a proof that, as $n \rightarrow \infty$, the moment generating function of $n^{1 / 2} \delta_{n}$ (for $F \in C$ ) converges to that of the limiting d.f. of $n^{1 / 2} \delta_{n}$. (Received May 26, 1955.)

817t. Jack Kiefer and Jacob Wolfowitz: On the characteristics of the general queueing process, with applications to random walk.

This paper continues the study of the general queueing process (many servers, arbitrary distributions of service time and time between successive arrivals) initiated by the authors in Trans. Amer. Math. Soc. vol. 78 (1955) pp. 1-18, the notation of which paper is used here. A typical result in the case $\rho<1$ where a limiting distribution function (d.f.) exists (the case $\rho \geqq 1$ is trivial) is that $\lim _{n \rightarrow \infty} n^{-1} \sum_{i=1}^{n}\left(w_{i 1}\right)^{k}=m_{k}$ with probability one, where $m_{k}(k>0)$ is the $k$ th moment of the limiting d.f. of the waiting time $w_{i 1}$, with analogous results for the mean queue length and mean busy period. Necessary and sufficient conditions for the finiteness of various moments are given, with the following random walk result as a special case: Let $x_{1}, x_{2}, \cdots$, be independent and identically distributed random variables with $-\infty<E x_{1}<0$. Let $S_{0}=0$ and $S_{n}=\sum_{1}^{n} x_{i}$. Let $v=\sup _{n} \geqq 0, S_{n}$. Then for any positive $k, E v^{k}<\infty$ if and only if $E\left(x_{1}^{+}\right)^{k+1}$ $<\infty$. (Received May 26, 1955.)

\section{8t. Seymour Sherman: $A$ theorem on convex sets with statistical ap-} plications.

Let $C_{0}$ be the closed convex cone generated in the uniform norm (that is, $\|f\|$ $=\sup \{|f(x)|\})$ by the characteristic functions of symmetric, compact, convex sets in real $n$ space, $\mathcal{E}$. Let $\mathcal{C}_{1}$ be the closed convex cone generated in the $L_{1}$ norm (i.e. $\|f\|_{1}$ $\left.=\int|f(x)| d x\right)$ by the characteristic functions of symmetric, compact, convex sets in $\mathcal{E}$. Consider the norm \|\|$_{3}$ given by $\|f\|_{3}=\max \left\{\|f\|,\|f\|_{1}\right\}$. Let $\mathcal{C}_{3}$ be the closed convex cone generated in the \|\|$_{3}$ norm by the characteristic functions of symmetric, compact, convex sets in $\mathcal{E}$. Note that for $E, K$ compact, convex and symmetric, the convolution of $\chi_{K}$ and $\chi_{E}$, the characteristic functions of $K$ and $E$ respectively, evaluated at $y$ becomes $\chi_{K} * \chi_{E}(y)=\int \chi_{E}(x) \chi_{E}(y-x) d x$. THEOREM 1. $\bigodot_{1} * \bigodot_{1} \subset_{C_{1}}$ and $\bigodot_{3} * C_{3} \subset C_{3}$. THEOREM 2. Let $E$ be a convex set in $\mathcal{E}$, symmetric about the origin. Let $f$ be a non-negative function such that (i) $f(x)=f(-x)$ for each $x \in \mathcal{E}$, (ii) $\{x \mid f(x) \geqq u\}$ $=K_{u}$ is convex for each $u, 0 \leqq u<\infty$, and (iii) $\int_{E} f(x) d x<\infty$, then $\int_{E} f(x+y) d x \in \bigodot_{3}$. This is a little stronger than a theorem of Anderson [The integral of a symmetric unimodal function over a symmetric convex set and some probability inequalities, Proc. Amer. Math. Soc. vol. 6 (1955) p. 170] and in fact the proof uses ideas in his proof but squeezes additional conclusions from the argument. The paper also gives a generalization to the multivariate case (with slight weakening of the symmetry assumptions in the hypothesis even in the univariate case) of results of $\mathrm{Z}$. W. Birnbaum [On random variables with comparable peakedness, Ann. Math. Statist. vol. 19 (1948) pp. 176-181]. (Received July 8, 1955.)

819. F. L. Spitzer: On the maximum of partial sums of random variables.

Let $\left\{X_{k}\right\}$ be a sequence of identically distributed random variables and $\left\{S_{k}\right\}$ the sequence of their successive partial sums. $S_{k}^{+}$is taken to equal $S_{k}$ if $S_{k} \geqq 0$ and zero otherwise. The characteristic functions of the random variables $S_{k}^{+}$are called $\psi_{k}(\lambda)$ 
and those of the random variables $\max _{1 \leqq k \leqq n} S_{k}^{+}$are called $\phi_{k}(\lambda)$ for $k \geqq 1$, while $\phi_{0}(\lambda)=1$. Then it follows from certain very general combinatorial identities for partial sums of real numbers that the following relation holds. For $|t|<1, \sum_{0}^{\infty} \phi_{n}(\lambda) t^{n}$ $=\exp \sum_{1}^{\infty}\left(\psi_{k}(\lambda) / k\right) t^{k}$. This result generalizes apparently dissimilar identities discovered by E. Sparre Andersen and M. Kac. (Received July 19, 1955.)

\section{0t. F. L. Spitzer: Some limit theorems for random variables.}

Let $X_{1}, X_{2}, \cdots$ be identically distributed independent random variables with distribution $F(x)$, and $S_{n}=X_{1}+\cdots+X_{n}, a_{n}=\operatorname{Pr}\left\{S_{n}>0\right\}, p_{n}=\operatorname{Pr}\left\{S_{1}>0, S_{2}>0\right.$, $\left.\cdots, S_{n}>0\right\}$ for $n \geqq 1$ and $p_{0}=1$. E. Sparre Andersen (Math. Scand. vol. 2 (1954) pp. 195-223) proved that $\sum_{0}^{\infty} p_{n} t^{n}=\exp \left(\sum_{1}^{\infty}\left(a_{n} / n\right) t^{n}\right)$ for $|t|<1$. The next three theorems follow from his result. I: $\operatorname{Pr}\left\{\sup _{n} \geqq 1 S_{n}>0\right\}=1-\exp \left(-\sum_{1}^{\infty} a_{n} / n\right)$. II: $\operatorname{Pr}\left\{S_{n}>0\right.$ i.o. $\}=\operatorname{Pr}\left\{\lim \sup _{n \rightarrow \infty} S_{n}=+\infty\right\}=1$ or 0 according as $\sum_{1}^{\infty} a_{n} / n=\infty$ or $<\infty$. III: $\sum_{1}^{\infty}(1 / n) \operatorname{Pr}\left\{\left|S_{n} / n-m\right|>\epsilon\right\}<\infty$ for every $\epsilon>0$ if and only if $\int_{-\infty}^{\infty} x d F(x)$ $=m$. (Received July 15, 1955.)

\section{TOPOLOGY}

\section{1t. F. W. Anderson: Ordered systems of regular open sets.}

Define the transitive binary relation « on the family $R(X)$ of all regular open subsets of the completely regular space $X$ by $U \ll V$ in case $U$ and the complement of $V$ are completely separated in $X$. Shirota (Osaka Math. J. vol. 4 (1952) pp. 121-132) proved that if $X$ is locally compact and $B C R(X)$ consists of those regular open sets with compact closure, then the system $(B, \ll)$ characterizes $X$. Using Shirota's result it is proved that the system $(R(X), \ll)$ characterizes the Stone-Cech compactification $\beta X$. In view of Tukey's generalization of Urysohn's Lemma (Convergence and uniformity in topology, Princeton, 1940, p. 53) this provides a completely "internal" construction of $\beta X$. It should be noted that, in general, $(R(X), \ll)$ fails to characterize the $Q$-space $\nu X$ (cf. Hewitt, Trans. Amer. Math. Soc. vol. 64 (1948) p. 88). Finally, it is observed that an arbitrary basis $B C R(X)$ need not characterize $\beta X$, but if $X$ is metrizable and $B$ contains all bounded $U$ in $R(X)$, then $(B, \ll)$ characterizes $X$. (Received July 18,1955 .)

\section{R. W. Bagley: A note on topologies on 2 R.}

The following topologies on $2 R$ are considered; the sequential order topology, $\tau_{1}$; the order topology, $\tau_{2}$; the topology of pointwise convergence, $\tau_{3}$ (here we consider $2 R$ as the cartesian product "R" times of $\{0,1\}$ with the discrete topology on $\{0,1\}$ ); and the interval topology, $\tau_{4}$ (the closed intervals are taken as a sub-basis for the closed sets). It is shown that when $R$ is countable $\tau_{1}=\tau_{2}=\tau_{3}=\tau_{4}$. However, when $R$ is uncountable $\tau_{1}<\tau_{2}<\tau_{3}=\tau_{4}$, where $\tau \leqq \tau^{\prime}$ means that every set open under $\tau^{\prime}$ is also open under $\tau$. A partial solution to problem 76 (Birkhoff, Lattice theory) is given (see also Northam, Proc. Amer. Math. Soc. (1953) pp. 824-827). (Received June 3, 1955.)

\section{3t. B. J. Ball: A note on the separability of an ordered space.}

It is shown that a connected ordered space $S$ is separable provided it satisfies Souslin's condition (there does not exist an uncountable collection of mutually exclusive open subsets of $S$ ) and there is a countable family $F$ of continuous functions of $S$ into itself such that each point $p$ of $S$ is a limit point of $\{f(p) \mid f \in F\}$. If $S$ is not required to satisfy Souslin's condition, the existence of such a family $F$ does not imply that $S$ is separable; however, if no element of $F$ has a fixed point or if the elements of $F$ can be arranged in a sequence $\left\{f_{n}\right\}$ such that for each point $p$ of $S,\left\{f_{n}(p)\right\} \rightarrow p$, 
then $S$ must satisfy Souslin's condition and hence must be separable. (Received July 19, 1955.)

\section{4t. R. H. Bing: Approximating wild surfaces with polyhedral ones.}

Suppose $S$ is a continuum in $E^{3}$ topologically equivalent to the surface of a sphere. It may be wild and its complement topologically different from the complement of a polyhedral surface. However, it is shown that for each positive number $\epsilon$, there is a polyhedral 2-sphere $S^{\prime}$ in $E^{3}$ and a homeomorphism $h$ of $S$ onto $S^{\prime}$ such that if $s \in S$, $\rho(s, h(s))<\epsilon$. The method used is to take a ruling of $E^{3}$ of small mesh, consider how $S$ intersects the boundaries of this ruling, and then construct the polyhedral surface $S^{\prime}$ near $S$ so that it intersects the boundaries of the ruling in a fashion approximating the way in which $S$ intersects the boundaries. Point set methods are used in the proof. (Received July 18, 1955.)

\section{R. L. Blair: A note on lattices of continuous functions. II.}

If $X$ is a $T_{1}$-space and $R$ a chain with its order topology, let $C(X, R)\left(C^{*}(X, R)\right)$ be the lattice of all continuous (bounded) functions from $X$ to $R$. If $\alpha, \beta \in R$, then $\alpha \ll \beta$ if (i) $\alpha \leqq \beta$ and (ii) either $\alpha<\beta$ or $\beta$ is minimal in $R$; dually for $\alpha \gg \beta$. Consider the following conditions on a sublattice $B$ of $C(X, R): \mathrm{C}_{1}$. Each prime ideal of $B$ is associated (cf. Kaplansky, Bull. Amer. Math. Soc. vol. 53 (1947) p. 618) with some $x \in X$. $C_{2}$. For $f, g \in B$ and disjoint closed sets $F, G$ of $X$ there is an $h \in B$ with $h(x) \ll f(x)$, $h(y) \gg g(y)$ for all $x \in F, y \in G$. $C_{3}$. Same as $\mathrm{C}_{2}$ except $F, G$ are required only to be distinct points of $X . \mathrm{C}_{4}$. For each prime ideal $P$ of $B$, if $P$ is associated with $x, y$, then $f(x)=f(y)$ for all $f \in B$. If $B$ satisfies $C_{1}$ then $B$ is characterizing (c-characterizing, wcharacterizing) if $B$ also satisfies $\mathrm{C}_{2}\left(\mathrm{C}_{3}, \mathrm{C}_{4}\right)$. Generalizing Kaplansky's Theorem 1 (loc. cit.) one has: If $B$ is a characterizing sublattice of $C(Y, R)$ then $Y$ is a continuous (1-1 continuous, homeomorphic) image of $X$ if and only if $B$ is isomorphic to a wcharacterizing ( $c$-characterizing, characterizing) sublattice of $C(X, R)$. For $X$ completely regular and $R=$ reals, constructions of $\beta X$ (Stone-Cech compactification) are given in terms of $C(X, R)$ and $C^{*}(X, R)$. For $X$ normal, characterizations of compactness in terms of $C(X, R)$ are also obtained. (Received July 18, 1955.)

\section{6t. C. E. Burgess: $A$ note on the separation of connected sets by} finite sets.

An elementary proof is presented to show that if $n$ is an integer greater than two and every set consisting of $n$ points separates the connected topological space $M$, then some set consisting of $n-1$ points separates $M$. This theorem was first proved for plane continuum by J. R. Kline [Closed connected sets which are disconnected by the removal of a finite number of points, Proc. Nat. Acad. Sci. U.S.A. vol. 9 (1923) pp. 712], and G. T. Whyburn has obtained a stronger result for locally compact metric continua [Local separating points of continua, Monatshefte für Mathematik und Physik vol. 36 (1929) pp. 305-314]. (Received July 18, 1955.)

827. C. E. Capel (p) and W. L. Strother: Traces for multi-valued functions.

Let $S(X)$ denote the space of non-null, closed subsets of a compact, Hausdorff space $X$. If $A \subset S(X)$ then a continuous function $s: \mathcal{A} \rightarrow X$ is a selection on $\mathcal{A}$ if $s(A) \in A$, for $A$ in $\mathcal{A}$. E. Michael (Topologies on spaces of subsets, Trans. Amer. Math. Soc. vol. 71 (1951) pp. 152-182) has shown there is a selection on $S(X)$ if $X$ is linearly ordered in a continuous sense. This idea is extended to finding selections on subsets of 
$S(X)$ when $X$ has a continuous partial order (for definition see L. E. Ward, Jr., Partially ordered topological spaces, Proc. Amer. Math. Soc. vol. 5 (1954) pp. 144161). It can then be shown, for a suitable space $X$ such as an $n$-cell or Tychonoff cube, that a continuous multi-valued function $F: X \rightarrow X$, with restrictions on the image of a point such as point-convex, has a trace and hence a fixed point. (Received July 8,1955 .)

\section{8t. W. H. Cockcroft: The cohomology groups of certain fibre spaces.}

Let $E$ be a fibre space, with fibre $F$ a space of type $K(\pi, n)$, base space $B$ simply connected, and characteristic class (minus transgression of fundamental class $u^{n}$ of fibre) $k^{n+1} \in H^{n+1}(B ; \pi)$. Standard spectral sequence arguments yield the following results. For a field $A$ of coefficients, $H^{i}(E ; A) \approx H^{i}(B ; A), 0 \leqq i \leqq n-1 ; H^{n}(E ; A)$ is a group extension, of the subgroup $\left\{\rho \in \operatorname{Hom}(\pi, A) \mid \rho k^{n+1}=0\right\} \subset \operatorname{Hom}(\pi, A)$, by $H^{n}(B ; A) ; H^{n+1}(E ; A) \approx H^{n+1}(B ; A) /\left\{\rho k^{n+1} \mid \rho \in \operatorname{Hom}(\pi, A)\right\}$; there is a composition series $H^{n+2}(E ; A) \supset D_{1} \supset D_{2} \supset 0$, where $H^{n+2}(E ; A) / D_{1} \approx\left\{S q^{2} \rho u^{n} \in H^{n+2}(F ; A) \mid \rho\right.$ $\in$ Hom $\left.(\pi, A), S q^{2} \rho k^{n+1}=0\right\}, \quad D_{1} / D_{2} \approx\left\{\sum b^{2} \otimes \rho u^{n} \in H^{2}(B ; A) \otimes H^{n}(F ; A) \mid b^{2}\right.$ $\left.\in H^{2}(B ; A), \rho \in \operatorname{Hom}(\pi, A), \sum b^{2} \cup_{\rho k^{n+1}}=0\right\}$, and $D_{2} \approx H^{n+2}(B ; A)$. In case $B$ is of type $K\left(\pi^{\prime}, n^{\prime}\right), n>n^{\prime}>1$, (and thus $E$ has two nonvanishing homotopy groups $\pi, \pi^{\prime}$ ) these results extend those obtainable from the relevant Eilenberg-MacLane theorem (Theorem IV, Ann. of Math. vol. 51 (1950) pp. 514-533). (Received June 9, 1955.)

\section{A. H. Copeland, Jr.: Binary operations on sets of mapping classes.}

Let $\pi(X ; Y)$ be the set of homotopy classes of maps $f: X \rightarrow Y$, where $X, Y$ are topological spaces, topological pairs, etc. If $X$ is a space of Lusternik-Schnirelmann category 2, $x_{0} \in X$, then for all spaces $Y$ and $y_{0} \in Y$ the set $\pi\left(X, x_{0} ; Y, y_{0}\right)$ has a binary operation such that the class of the constant map is a (2-sided) identity and a map $g: Y, y_{0} \rightarrow Y^{\prime}, y_{0}^{\prime}$ induces a natural homomorphism $g_{\sharp}: \pi\left(X, x_{0} ; Y, y_{0}\right) \rightarrow \pi\left(X, x_{0} ; Y, y_{0}\right)$. The converse to this statement is proved. A similar study is made for the case $Y$ fixed and $X$ arbitrary. Here, the existence of a binary operation is related to $Y$ being an $H$-space. As applications, a new proof that a contractible fibre is an $H$-space is found, and it is proved that if $X$ is a suspended space, $x_{1}, \cdots, x_{r} \in H^{n}(X ; G), y \in H^{q}(G, n ; H)$ then the Eilenberg-MacLane product $\left[x_{1}, \cdots, x_{r} ; y\right]=0 \in H^{q}(X ; H)$. (Received July $5,1955$.

830. H. S. M. Coxeter: Abstract definitions for the plane crystallographic groups.

The 17 two-dimensional space-groups (i.e., the 17 ways of repeating a flat design, as on wallpaper) were discovered empirically by the Moors in their decoration of the Alhambra. The first mathematical treatment was by Fricke and Klein (Vorlesungen über die Theorie der automorphen Funktionen, Leipzig, 1897). The groups were rediscovered by Pólya and Niggli (1924) and were shown by Nowacki (Schweiz. Min. Petr. Mitt. vol. 34 (1954) pp. 160-168) to be abstractly distinct. The present paper gives an abstract definition for each group in terms of two, three, or four generators. The two cases in which a single relation suffices have a connection with topology: $X Y=Y X$, generated by two translations, and $P^{2}=Q^{2}$, generated by two parallel glide-reflections, are the fundamental groups of the torus and the Klein bottle, respectively. (Received July 15, 1955.)

831. M. L. Curtis: The covering homotopy theorem. 
Let $E$ and $B$ be spaces and $p: E \rightarrow B$ be a map. A test space $C$ is constructed such that if $(E, B, p)$ satisfies the covering homotopy theorem for the space $C$, then it satisfies the covering homotopy theorem with respect to any topological space. For $(E, B, p)$ a locally trivial fiber space, Huebsch has proved that one has the covering homotopy theorem for paracompact spaces. So, for example, if $(E, B, p)$ is a locally trivial fiber space and $E$ and $B$ are metric, then one has the covering homotopy theorem for arbitrary spaces. (Received July 5,1955 .)

\section{M. L. Curtis and R. K. Lashof (p): Homotopy equivalence of fiber bundles.}

For a fiber bundle with fiber $F$, an associated fiber space with an $H$-space $M$ as fiber is constructed. $M$ consists of all maps of $F$ into $F$ which have homotopy inverses. These associated spaces play the same role for fiber homotopy equivalence that associated principal bundles play for bundle equivalence. Bundles are also classified according to fiber homotopy equivalence by a map of the loops of the base into the $H$-space $M$. It is known that any two tangent sphere bundles of a differentiable manifold are fiber homotopy equivalent, and the above theory is applied to this situation to show that some characteristic classes are independent of the differential structure. (Received July 13, 1955.)

\section{W. F. Davison: An equivalence relation for compact Hausdorff} varieties.

The basic structure is a compact Hausdorff space $X$ and a class $F$ of functions from $X$ into a set $Y$, with the compatibility condition: for all $f, g \in F$, if $E$ in $X$ is closed then $g^{-1} f(E)$ is closed. A subset $g(X)$ of $Y$ is topologized by defining $G$ in $g(X)$ to be closed if and only if $g^{-1}(G)$ is closed; this topology is compact Hausdorff and $g$ is closed and continuous. If $f(X) \subset g(X)$ then $f$ is closed and continuous. If $\Phi$ is the unique uniformity for $X$ then for $U \in \Phi$ and $x \in g(X), g U g^{-1}(x)$ is a neighborhood of $x$. The collection of relations $\{V(U): U \in \Phi\}$, where $V(U)=g U g^{-1} g U g^{-1}$, is a basis for the unique uniformity for $g(X)$. Define $f \sim g$ if and only if for every $U \in \Phi$ there is a homeomorphism $h: X \rightarrow X$ such that $f h \subset V(U) g$. Then $\sim$ is an equivalence relation and $f \sim g$ implies $f(X)=g(X)$. If $Y$ is topologized so that every compact set is closed then the continuous functions are compatible and the $Y$ topology relativized to $g(X)$ agrees with the above topology. If $X$ is Peanean and $Y$ metric, then continuous functions are -equivalent if and only if they are Frechét equivalent. (Received July 18, 1955.)

\section{D. O. Ellis: The topology of finitary approximation.}

A topology previously employed ( $R$. Vaidyanathaswami, Treatise on set topology I, Madras, Indian Mathematical Society, 1947, p. 154) in the space of mappings of an arbitrary set into the unit interval is generalized to the case of an arbitrary metric space as range and is compared with the topologies induced by pointwise and uniform convergence. The methods are metric and topolattice-theoretic (Robert Bagley and David Ellis, On the topolattice and permutation group of an infinite set, Mathematica Japonicae vol. 3 (1954) pp. 63-70). (Received June 29, 1955.)

\section{M. K. Fort, Jr.: Extensions of mappings into n-cubes.}

Let $X$ be an $m$-dimensional separable metric space, and let $h$ be a mapping on a closed subset $K$ of $X$ into the closed $n$-cube $I^{n}, m \geqq n>0$. It is proved that $h$ can be extended to a mapping $f$ on $X$ into $I^{n}$ such that $\operatorname{dim} f^{-1}(y) \cap(X-K) \leqq m-n$ for each 
$y \in I^{n}$. It is also shown that if $m \geqq n>0$ and $X$ is a compact metric space, then $\operatorname{dim} X$ $\leqq m$ if and only if there exists a mapping $f$ on $X$ into $I^{n}$ such that $\operatorname{dim} f^{-1}(y) \leqq m-n$ for each $y \in I^{n}$. For the case $m=n$, this last result was obtained by M. L. Curtis and G. S. Young in $A$ theorem on dimension, Proc. Amer. Math. Soc. vol. 3 (1952) pp. 159161. (Received June 6, 1955.)

836. R. E. Fullerton: The Carathéodory mapping theorem for twodimensional manifolds.

Let $M$ be a compact two-dimensional manifold with boundary and $G$ an open subset of $M$ with a finite number of boundary components. Ends and prime ends of $G$ can be defined in a manner similar to that used for simply connected plane regions. Certain ordered segments of ends and prime ends are defined and by rectifying these segments it is shown that there exists a mapping of $\bar{G}$ onto a subset $K \subset M$ which is a two manifold with boundary. This mapping is a piecewise linear homeomorphism on the interior of $\bar{G}$ and defines a homeomorphism between segments of ends and prime ends of $G$ and boundary components of $K$ where the order topology is used on the segments. (Received July 18, 1955.)

\section{7t. C. D. Gorman: $A$ note on recurrent flows.}

The following theorem is proved which answers a question by NemyckiY concerning a characterization of pseudominimal sets: if $f$ is a continuous flow in a metric space $R$, consider the statements: (I) $f$ is recurrent; (II) there exists a sequence $t_{1}<t_{2}<\cdots \rightarrow \infty$ of real numbers such that $\lim _{n \rightarrow \infty} f\left(A, t_{n}\right)=A$ for every closed subset $A$ of $R^{\prime}$, (III) there exists a sequence $t_{1}<t_{2}<\cdots \rightarrow \infty$ of real numbers such that $\lim \sup _{n \rightarrow \infty} f\left(A, t_{n}\right) \subset A$ for every closed subset $A$ of $R$; then (1) I implies II; II implies III, (2) if $R$ is compact, then I, II, III are equivalent. (Received June 22, 1955.)

\section{Alexandre Grothendieck: On Stein manifolds.}

Let $X$ be a connected Stein manifold of dimension $n \geqq 2$, and $Y$ any Stein manifold. A holomorphic function on a connected neighborhood $\mathcal{U}$ of $\infty$ in $X$, with values in $Y$, extends holomorphically over $X$ (this result is well known if $Y$ is the complex field $C$ ). Hence the analogous result for holomorphic sections of an analytic fibre bundle $E$ over $X$, with fibre $Y$, holds true if we know that $E$ is Stein, for instance, if $E$ is principal with linear complex structure group $Y$. Hence, if such a bundle is trivial on a neighborhood of $\infty$, it is trivial throughout. Interesting information on the fundamental group of $X$, including $H_{1}(\mathcal{U}, Z) \rightarrow H_{1}(X, Z)$ is onto, is obtained by taking $Y$ as a discrete linear group. Another example: $Y=C^{*}$ (multiplicative group of $C$ ), then the preceding result becomes $H_{*}^{1}\left(X, 0 C^{*}\right)$ (cohomology with compact supports, coeffcients in the bundle of germs of nonvanishing holomorphic functions), which classically means: $H_{*}^{2}(X, Z)=0$, that is $H_{2 n-2}(X, Z)=0$ for $n \geqq 3, H_{*}^{2}(X, Z)=H_{2}(X, Z)$ without torsion if $n=2$ ( $Z=$ integers). (The corresponding result for Betti numbers is known.) (Received May 2, 1955.)

\section{Mario Gutiérrez-Burzaco: Uniform homotopy groups.}

Let $X, Y$ be uniform spaces, and $I=[0,1]$. A uniformly continuous map (abbreviated u-map) $h: X \times I \rightarrow Y$ is called a uniform homotopy (u-homotopy). Consider an arcwise connected subset $A \subset Y$ and a base point $y_{0} \in A$. Let $I^{n}$ be the unit $n$-cube in some Euclidean space, and let $\dot{I}^{n}$ be its boundary. For $0<p<n$ let $I^{n, p}=I^{n}-\left\{x \in I^{n}\right.$ $\left.\mid 0<x_{i}<1,1 \leqq i \leqq p ; x_{j}=1 / 2, p<j \leqq n\right\}$. The set of classes of u-homotopic u-maps $f:\left(I^{n, p}, \dot{I}^{n}\right) \rightarrow\left(A, y_{0}\right)$ forms a group denoted by $\pi_{n, p}(A)$. Let $I_{-}^{n, p}=\left\{\left(x_{1}, \cdots, x_{n}\right)\right.$ 
$\left.\mid\left(x_{1}, \cdots, x_{n}+1\right) \in I^{n, p}\right\}$. Using $I^{n} \bigcup I^{n, p}$ as antecedent, one defines similarly the group $\pi_{n, p}(Y, A)$; for $0<p<n$ these are "intermediate" between the usual relative homotopy group $\pi_{n}(Y, A)$ and the absolute h. g. $\pi_{n}(Y)$. The following homomorphisms are then defined among the above four types of groups: $\partial: \pi_{n+1, p+1}(Y, A) \rightarrow \pi_{n}(A)$, $i: \pi_{n}(A) \rightarrow \pi_{n}(Y), j: \pi_{n}(Y) \rightarrow \pi_{n, p}(Y), k: \pi_{n, p}(A) \rightarrow \pi_{n, p}(Y, A), l: \pi_{n}(A) \rightarrow \pi_{n, p}(A)$. For each positive $n-p$ a branched sequence can be formed thus: upper branches: $\cdots, \partial$, $i, j, \partial, \cdots ;$ lower branches: $\cdots, \partial, l, k, \partial, \cdots$. The following "exactness" properties hold: (i) $\partial=i^{-1}(0) \cap l^{-1}(0)$, (ii) $\partial^{-1}(0)=j+k$ (sum of complexes), (iii) $i=j^{-1}[k]$, (iv) $l=k^{-1}[j]$, (v) $j^{-1}(0)=i\left[l^{-1}(0)\right]$, (vi) $k^{-1}(0)=l\left[i^{-1}(0)\right]$, where $\partial$ stands for the image of $\partial$, and similarly for the other homomorphisms. The proof uses an extension theorem for u-homotopies defined on uniform absolute neighborhood retract (u-ANR) subsets of u-ANR metric spaces. (Received June 30, 1955.)

840. Frank Harary: The number of dissimilar supergraphs of a linear graph.

Two subgraphs of a linear graph are similar if there exists an automorphism sending one onto the other. A $(p, q)$ graph is one with $p$ vertices and $q$ lines. A formula is obtained for the number of dissimilar occurrences of a given $(a, b)$ graph $G$ as a subgraph of all $(p, q)$ graphs, $a \leqq p, b \leqq q$, i.e., for the number of dissimilar $(p, q)$ supergraphs of $G$. The enumeration methods are those of P6lya, Acta Math. vol. 68 (1937) pp. 145-254. This result is then applied to obtain formulas for the number of dissimilar cycles and the number of dissimilar complete subgraphs (cliques) among all $(p, q)$ graphs. The formula for the number of rooted graphs obtained in Trans. Amer. Math. Soc. vol. 78 (1955) pp. 445-463 is a special case of the number of dissimilar cliques. This note complements The number of dissimilar line-subgraphs of a given graph, Pacific Journal of Mathematics (to appear) in which the number of dissimilar $(p, k)$ subgraphs of a given $(p, q)$ graph is obtained. Unsolved problem. What is the number of dissimilar Hamilton lines in an $n$-cube? And in general, how many dissimilar times does a fixed graph $H$ occur as a subgraph of a fixed graph $G$ ? (Acknowledgment: This work was supported by the Bell Telephone Laboratories, Summer 1955.) (Received July $14,1955$.

\section{L. J. Heider: Directed limits on rings of continuous functions.}

Let $\beta X$ be the collection of all maximal proper dual ideals $\alpha$ of subsets of set $X$. Real valued function $f$ on $X$ has a directed limit at $\alpha$ with value $\bar{f}(\alpha)$ provided a number $\bar{f}(\alpha)$ exists such that, for every $\epsilon>0,|\bar{f}(\alpha)-f(x)|<\epsilon$ for all $x$ in some subset of $X$ in $\alpha$. Every real bounded function on $X$ has a unique directed limit at every $\alpha$. Hausdorff quotient spaces of $\beta X$ as under the Wallman topology together with the $\bar{f}$ determine $X_{T}, \beta X_{T}, v X_{T}, C^{*}\left(X_{T}\right), C\left(X_{T}\right), C\left(\beta X_{T}\right)$ and $C\left(v X_{T}\right)$ for every completely regular topology $T$ on set $X$. Here $\beta X_{T}$ and $v X_{T}$ denote the Čech compactification and the Hewitt $Q$-space associated with $X_{T}$. Maximal ideals of $C\left(X_{T}\right)$ consist of elements $f$ with zero sets $Z(f)$ in some $\alpha$ contained in a given maximal collection of $\alpha$ identified on $C^{*}\left(X_{T}\right)$. The real maximal ideals of $C\left(X_{T}\right)$ consist of elements $f$ with $\bar{f}(\alpha)=0$ on a given $\alpha$ such that $\bar{f}(\alpha)$ exists for all $f$ in $C\left(X_{T}\right)$. Alternatively, they consist of elements with $\bar{f}(\alpha)=0$ on a given $\alpha$ such that $\bar{f}(\alpha)=0$ for an $f$ in $C^{*}\left(X_{T}\right)$ if and only if $Z(f)$ is nonempty. (Received July 13, 1955.)

842. Erik Hemmingsen: Some regular homeomorphisms in three space.

Let $M$ be a continuum which is the closure of an open set in $E^{3}$ and let $f$ be a regu- 
lar homeomorphism of $M$ onto itself, i.e., one whose iterates form an equicontinuous family. If there is a point of $M$ whose orbit closure (under the iterates of $f$ ) is connected, then the orbit closures of all the non-fixed points of $M$ must be either simple closed curves or the product of two simple closed curves. (Received June 20,1955.)

843t. V. L. Klee, Jr.: Some finite-dimensional affine topological spaces.

In Euclidean $m$-space $E^{m}$ with $m \geqq 2$, the following enlargements of the usual topology are considered: $\left(T_{1}\right)$ A subset $X$ of $E^{m}$ is $T_{1}$-open if and only if each point of $X$ is radially interior to $X$. $\left(\mathrm{T}_{2}\right)$ A subset $X$ of $E^{m}$ is $\mathrm{T}_{2}$-open if and only if it can be expressed in the form $X=G \cup H$, where $G$ is an open subset of $E^{m}$ and each point $h$ of $H$ is radially interior to some closed subset of $G \cup\{h\}$. For each $i,\left(E^{m}, T_{i}\right)$ is an affine topological space in the sense of Fréchet, though not a linear topological space in the sense of Tychonoff. It is proved that $\left(E^{m}, \mathrm{~T}_{1}\right)$ is not regular, that $\left(E^{m}, \mathrm{~T}_{2}\right)$ is completely regular but not normal, and that for each $i,\left(E^{m}, \mathrm{~T}_{i}\right)$ is separable but lacks the Lindelöf property and fails to satisfy the first axiom of countability. (Received June 15, 1955.)

844t. S. D. Liao: Periodic transformations and fixed point theorems. I. Cup products and special cohomology.

The main aim of this paper is to introduce certain relationships which connect (i) the special cohomology modulo $p$ arising from a transformation of prime period $p$ acting on a space, and (ii) the multiplicative structure modulo $p$ of the cohomology of the space and the fixed point set. Let $T$ be a periodic transformation on a compact Hausdorff space $X$ of period $p$ with fixed point set $F$. Let $X_{0}$ be a closed subset of $X$, invariant under $T$. Certain relations, called "special relatedness," given by $T$ between a (finite) set $\Delta$ of cohomology classes over the pair $\left(F, X_{0} \cap F\right)$ and a cohomology class $\alpha_{q}$ over the pair $\left(X, X_{0}\right)$ are considered. Let $X_{0}^{\prime}$ be also a closed subset of $X$, invariant under $T$. If a set $\Delta_{1}$ of cohomology classes over $\left(F, X_{0} \cap F\right)$ is specially related to a cohomology class $\alpha_{q}$ over $\left(X, X_{0}\right)$ and if a set $\Delta_{2}$ of cohomology classes over $\left(F, X_{0}^{\prime} \cap F\right)$ is specially related to a cohomology class $\beta_{r}$ over $\left(X, X_{0}^{\prime}\right)$, then special relatedness exists between $\Delta_{1} \bigcup * \Delta_{2}$ and $\lambda \alpha_{q} \bigcup_{\beta_{r}}$ in most cases, where $\Delta_{1} \bigcup_{* \Delta_{2}}$ con. sists of certain linear combinations of cohomology classes $\gamma_{1} \cup_{\gamma_{2}}$ over $\left(F,\left(X_{0} \cup X_{0}^{\prime}\right)\right.$ $\cap F)$ with $\gamma_{1}$ in $\Delta_{1}, \gamma_{2}$ in $\Delta_{2}$, and $\lambda$ is \pm 1 or $\pm(1-T)^{p-2}$. The result is also generalized to relations concerning local special cohomology groups ${ }^{\rho} H^{i}\left(X_{0}\left|X,\left(X_{0} \cap F\right)\right| F ; Z_{p}\right)$ etc. and local cup products $H^{i}\left(X_{0}, Z_{p}\right) \cup H^{i}\left(X_{0} \mid X, Z_{p}\right) \rightarrow H^{i+i}\left(X_{0} \mid X, Z_{p}\right)$ etc. (Received July 25, 1955.)

845t. S. D. Liao: Periodic transformations and fixed point theorems. II. Manifolds. Preliminary report.

Let $M^{n}$ be an $n$-dimensional compact connected, orientable manifold, and let $X_{0}$ be a closed subset of $M^{n}$. The Poincaré-Alexander duality in $M^{n}$ can be inversed in terms of local cup or cap products between the cohomology or homology over $X_{0}$ and those in the neighborhoods of $X_{0}$ in $M^{n}$. The results in the previous paper (Periodic transformations and fixed point theorems. I) are applied to study the homology properties of fixed point sets of periodic transformations acting on manifolds. The main result of this paper is the following "torsion abelian group" theorem: The fixed point set of a torsion abelian group of differentiable transformations of class $C^{\infty}$ acting on a sphere is a homology sphere over rationals. Other results are also established. For instance: Let $T$ be a periodic transformation on an $n$-sphere $S^{n}$ of prime period $p$. 
Let $M^{q}, N^{r}$ be respectively $q$-dimensional and $r$-dimensional compact connected orientable submanifolds in $S^{n}$ with $q+r=n-1$. Suppose that $M^{q}$ and $N^{r}$ are disjoint, invariant under $T$, and linked modulo $p$. Then, if $M^{q}$ contains no fixed points of $S^{n}$, $N^{r}$ contains all the fixed points of $S^{n}$. (Received July 25,1955 .)

\section{G. R. Livesay: Involutions on a manifold.}

Let $T: M \rightarrow M$ be a simplicial mapping of the triangulated compact manifold $M$ onto itself, with no fixed points. The Smith-Richardson homology theory shows that there exists a "fundamental domain," $\left|C_{n}\right|$, of $T$ satisfying (a) $C_{n}+T C_{n}$ is the fundamental $n$-cycle on $M$, (b) $\partial C_{n}$ is a $T$ invariant $n-1$ cycle not $T$-homologous to zero, (c) $C_{n}$ is $n-1$ connected, (d) if $A$ and $B$ are closed subcomplexes of $M$ such that $A \cup B=M$, and no component of $A$ contains an involution pair, then $\partial C_{n} \subset B$. If, in addition, $M$ is unicoherent, and $T_{1}$ and $T_{2}$ are isometric involutions, then it follows that the boundaries of the corresponding fundamental domains must intersect. This result implies the following conjecture of M. H. Steinhaus and B. Knaster [Colloquium Mathematicum 1, 1947-1948]: Let $f: S_{2} \rightarrow E_{1}$ be continuous, $p_{1}, p_{2}, p_{3}$ any three points of $S_{2}\left(=2\right.$-sphere). Then there exists a rotation $r$ of $S_{2}$ such that $f\left(r p_{1}\right)=f\left(r p_{2}\right)=f\left(r p_{3}\right)$. The manifold $M$, in this application, is $S O(3) . T_{1}$ and $T_{2}$ are the involutions obtained by right multiplication by $r_{2}$ and $r_{3}$, where $r_{i}$ is defined by $r_{i} p_{i}=p_{1}, r_{i}{ }^{2}=$ identity. (Received May 2, 1955.)

\section{7t. E. A. Michael: On the completion of a uniformly $L C^{n}$ or $A N R$} metric space.

Methods developed by J. Dugundji show that the results in $\$ 1$ of S. Eilenberg and R. L. Wilder [Amer. J. Math. vol. 64 (1942) pp. 613-622] are valid without separability restrictions. This implies, in particular, that if $Y$ is a dense subset of a metric space $Z$, and if $Y$ is uniformly $\mathrm{LC}^{n}$, then so is $Z$; in fact, if every continuous image of a $k$-sphere $(k \leqq n)$ in $Y$ of diameter $<\delta(\epsilon)$ is contractible over a subset of $Y$ of diameter $\langle\epsilon$, then the same is true for $Z$ with $\delta(\epsilon)$ replaced by $\delta(\epsilon / 2)$. The same methods yield analogous results for spaces $\gamma$ which are uniformly $\mathrm{ANR}_{\text {metrio }}$ (i.e. to every $\epsilon>0$ there corresponds a $\delta(\epsilon)>0$ such that every partial realization of a simplicial $\mathrm{CW}$-complex into $Y$ of mesh $<\delta(\epsilon)$ can be extended to a full realization of mesh $<\epsilon$ ). Combined with Theorems 1 and $1^{\prime}$ of J. Dugundji and E. Michael [To appear in Proc. Amer. Math. Soc.], this yields the following corollary. If a metrizable space $Y$ is $\mathrm{ANR}_{\text {metric }}$, or $\mathrm{AR}_{\text {metric, }}$ or $\mathrm{LC}^{n}$, or $\mathrm{LC}^{n}$ and $\mathrm{C}^{n}$, then so is the completion of $Y$ with some admissible metric $\rho$; moreover, $\rho$ can be chosen to be uniformly finer than any given admissible metric. (Received July 18, 1955.)

848t. E. A. Michael: On two extension theorems of Hanner and Dowker.

The corollary in the preceding abstract yields the following sharpening of two theorems of O. Hanner and C. H. Dowker, concerning the extension of continuous functions whose domain is normal or collectionwise normal: If $X$ is normal (resp. collectionwise normal), if $A \subset X$ is closed, and if $Y$ is a metric (resp. separable metric) ANR $_{\text {metric }}$ with completion $\bar{Y}$, then every continuous $f: A \rightarrow Y$ can be extended to a continuous $g: U \rightarrow \bar{Y}$ for some open $U \subset A$; if moreover $Y$ is an $\mathrm{AR}_{\text {metrio, one can take }}$ $U=X$. The $n$-dimensional analogues of the theorems of Hanner and Dowker (E. Michael, Bull. Amer. Math. Soc. Abstract 61-5-631) can be similarly sharpened; one then obtains the above result with $\mathrm{ANR}_{\text {metric }}$ replaced by $\mathrm{LC}^{n}$, and $\mathrm{AR}_{\text {metrio }}$ replaced 
by $C^{n}$, provided one also assumes that every closed (in $X$ ) subset of $X-A$ has Lebesgue dimension $\leqq n+1$. (Received July 18, 1955.)

849t. Deane Montgomery, Hans Samelson, and Leo Zippin: Singular points of a compact transformation group.

Let $G$ be a compact Lie group which acts on an $n$-dimensional manifold $M$. Let $s$ be the maximum of the dimensions of the orbits of $G$ in $M$; it is known that the union of all orbits of dimension $s$ is an open set $X$. It is proved now that the singular set, the union of all orbits of dimension less than $s$, has no interior points and is of dimension at most $n-2$; it follows that the set $X$ is connected. In more detail it is shown that the subset of the singular set, made up of orbits of any fixed dimension $s^{\prime}(<s)$, has dimension at most $n-1-\left(s-s^{\prime}\right)$. Finally it is shown that the orbit space $M^{*}$ (the orbits under $G$ considered as points) has dimension at most $n-s$. The proofs utilize the homology characterization of dimension and Gleason's construction of a cross section of an invariant set, if all the stability groups are conjugate to each other. (Received July 15, 1955.)

850t. P. S. Mostert and A. L. Shields: Differentiability in semigroups on manifolds with boundary.

Let $S$ be a compact manifold with connected boundary $B$. Let $S$ be a topological semigroup with identity, such that $B$ is a Lie group. A. D. Wallace has raised the question (Bull. Amer. Math. Society Research Problem 61-1-10) whether the multiplication may be assumed differentiable throughout all of $S$. This is in general false. In fact, it is true if and only if the quotient semigroup $S / B$ is the unit interval $[0,1]$ under ordinary multiplication. In general, one has differentiability in a neighborhood of any point $x$ such that $x^{2} B$ contains no idempotents. (Received June 13,1955 .)

851t. P. S. Mostert and A. L. Shields: On abelian semigroups which are topologically manifolds with boundary.

Let $S$ be a compact manifold with connected boundary $B$. Suppose $S$ is an abelian topological semigroup with identity, such that $B$ is a sub-semigroup. Then $B$ is a toroidal group, and $S$ is topologically and algebraically of the form: $D \times B$. Here $D$ is one of the following: I. If $S$ is orientable, then $D$ is a semi-group on the unit disc with boundary which is a circle group. II. If $S$ is non-orientable, then $D$ is a semigroup on the Moebius strip with boundary circle a circle group. (Received June 13, 1955.)

852. P. S. Mostert ( $\mathrm{p}$ ) and A. L. Shields: On the existence of oneparameter subsemigroups in topological semigroups.

Define an adequate local semigroup to be a Hausdorf space $S$ and a neighborhood $W$ in $S$ with the following properties: there is a continuous, associative multiplication defined for elements of $\bar{W}$ with products in $S$, there is an element $1 \in W$ such that $x 1=1 x=x$ for all $x \in \bar{W}$, and the set $H(1)=[x \in \bar{W}$ : there is a $y \in \bar{W}$ with $x y=y x=1]$ is a topological group contained in $W$. (In particular, a compact semigroup with identity is an adequate local semigroup.) Theorem: Let $S$ be an adequate local semigroup with $\bar{W}$ compact. Suppose, moreover, that $\bar{W}$ contains no idempotents except 1 and that $H(1)$ is a Lie group and is not an open set. Then there exists a continuous function $f:[0,1]$ to $\bar{W}$, such that $f(0)=1, f(a+b)=f(a) f(b)$ for $0 \leqq a+b \leqq 1, f$ is $1-1$, and $f(a)$ is not in $H(1)$ for $a \neq 0$. (Received June 13, 1955.) 
853. P. S. Mostert and A. L. Shields (p): On the structure of semigroups which are topologically manifolds with boundary.

Let $S$ be a compact manifold with connected boundary $B$. Let $S$ be a topological semigroup with identity, 1 , such that $B$ is a Lie group. Pierre Conner has shown that $K$, the minimal two-sided ideal of $S$, must be a group. Let $e$ be the idempotent in $K$. Then there is an $\operatorname{arc} J$ in $S$ from $e$ to 1 such that: (1) $J$ is a subsemigroup of the type described in a previous abstract on the unit interval, (2) $J$ is in the center of $S$, (3) $S=J B$, (4) if $J_{0}=J-\{e\}$, and $S_{0}=S-K$, then $J_{0} B, J_{0} \times B$, and $S_{0}$ are all isomorphic and homeomorphic under the natural map. This completely determines the topological and algebraic structure of $S$. (Received June 13, 1955.)

854t. P. S. Mostert and A. L. Shields: On topological semigroups on the unit interval.

Let $A=[a, b]$ be a closed interval with a continuous, associative multiplication such that: $a x=x a=a$ and $b x=x b=x$ for all $x$ ( $b$ is an identity and $a$ is a zero). If $A$ contains no other idempotents, then $A$ is topologically and algebraically isomorphic to one of the following: I. $[0,1]$ under ordinary multiplication; II. $[1 / 2,1]$ with the multiplication $x * y=\max [1 / 2, x y]$, where $x y$ denotes the ordinary product, and $*$ denotes the semigroup product. In the general case, let $E$ be the set of idempotents of $A$. Then $E$ is closed and contains $a$ and $b$. Its complement consists of disjoint open intervals $(c, d)$. The closure of each such interval is a subsemigroup of the form I or II. Finally, if we order $A$ in the usual way from left to right, and if $x, y$ are not in the same complementary interval of $E$, then $x * y=\min (x, y)$. (Monroe Faucett, Compact semigroups irreducibly connected between two idempotents, Proc. Amer. Math. Soc. vol. 6 (1955) pp. 741-747 has shown that $A$ must be abelian, and that if $A$ contains no idempotents other than $a$ and $b$, and has no nilpotent elements, then $A$ is $I$.) (Received June 13, 1955.)

\section{Mary E. Rudin: A separable normal non-paracompact space.}

It has been shown that every normal Hausdorff space with a countable base is paracompact. The purpose of this paper is to show that there is a separable, normal Hausdorff space that is not paracompact. (Received July 11, 1955.)

856. W. L. Strother (p) and C. E. Capel: Fixed point property for the space of subsets of an absolute retract.

Wojdyslawski [Retracts absolus et hyperspaces des continus, Fund. Math. vol. 32 (1939) pp. 184-192 ] asked "If $X$ is a CAR* (= retract of a cube) is the space of closed subsets $S(X)$ a CAR*?" The question has been answered in the affirmative [W. L. Strother, Fixed points, fixed sets, and $M$-retracts, Duke Math. J., to appear] for the special case in which $X$ is a Peano continuum. This result is used here to prove that if $X$ is a CAR* then $S(X)$ has the fixed point property. (Received July 22,1955.)

\section{Emery Thomas: A generalization of the Pontrjagin square cohomology operation.}

Let $K$ be a finite regular cell complex and $n$ an even integer. For each prime $p$ we define a function $\mathfrak{P}_{p}: H^{n}\left(K ; \bmod p^{r}\right) \rightarrow H^{p n}\left(K ; \bmod p^{r+1}\right)(r \geqq 1)$, such that $\mathfrak{P}_{2}$ is the Pontrjagin square cohomology operation. Each function $\mathfrak{P}_{p}$ is topologically invariant, and hence is a cohomology operation. Using the complex projective space of $2 p$ real 
dimensions, we construct an example of two complexes which known cohomology invariants fail to distinguish as to homotopy type, but which the operation $\mathfrak{B}_{p}$ does distinguish. The operation $\mathfrak{P}_{p}$ is then generalized to an operation $\mathfrak{P}_{t}(t=0,1, \cdots)$ such that $\mathfrak{P}_{t}: H^{n}(K ; \Pi) \rightarrow H^{t n}\left(K ; \Gamma_{t}(\Pi)\right)$, where $\Pi$ is a finitely generated abelian group and $\Gamma_{t}(\Pi)$ is a subgroup of the ring $\Gamma(I I)$ defined by Eilenberg and MacLane [Ann. of Math. vol. 60 (1954) p. 107]. The sequence of functions $\mathfrak{P}=\left[\mathfrak{P}_{t}\right]_{t=0}^{\infty}$ then satisfies the same set of relations as do the generators of the ring $\Gamma(\Pi)$ : namely, if $u \in H^{n}(K ; \Pi)$, (i) $\mathfrak{B}_{0}(u)=1$, the unit of the cohomology ring of $K$ with integer coefficients; $\mathfrak{B}_{1}(u)$ $=u$; (ii) $\mathfrak{B}_{r}(u) \cup \mathfrak{B}_{s}(u)=C_{r+s, r} \mathfrak{P}_{r+s}(u)$; (iii) $\mathfrak{P}_{t}(u+v)=\sum_{r+s=t} \mathfrak{B}_{r}(u) \cup \mathfrak{P}_{\mathrm{s}}(v)$, for $u, v \epsilon H^{n}(K ; \Pi)$. (Received July 7, 1955.)

858t. A. D. Wallace: One-dimensional homogeneous clans are groups.

The theorem of the title is proved.

\section{C. T. Yang: Mappings from spheres to euclidean spaces.}

The following theorem is proved: A mapping from an $(m n)$-sphere to the euclidean $m$-space maps the end points of some $n$ mutually orthogonal diameters into a single point. A Borsuk-Ulam's theorem (Fund. Math. (1933)), a Dyson's theorem (Ann. of Math. (2) (1951)) and some results of the author (Ann. of Math. (2) (1954) and Bull. Amer. Math. Soc. Abstract 60-6-547) are all included here as special cases. The proof of this theorem needs a generalized Borsuk-Ulam's theorem and a generalized Kakutani-Yamabe-Yujobô's theorem, established by the author (Ann. of Math. (2) (1954)), and the following lemma. Let $X$ be a compact subset of a real projective space $P$ such that the homomorphism $H_{m n+n}(X) \rightarrow H_{m n+n}(P)$, induced by the inclusion mapping, is not trivial, where homology groups are Cech groups with integers mod 2 as coefficients. Then for any mapping $f$ of $X$ into the euclidean $m$-space $E$ there is a point $y$ of $E$ such that the homomorphism $H_{n}\left(f^{-1}(y)\right) \rightarrow H_{n}(P)$ is not trivial. (Received July 15,1955 .)

\section{J. A. Zilber: Type-complexes. Preliminary report.}

Let $\left\{\pi_{n}\right\}$ denote a sequence of groups, $n=1,2, \cdots$, abelian for $n>1$, together with an operation of $\pi_{1}$ on each $\pi_{n}, n>1$. For any complete semi-simplicial (css) complex $K$, let $|K|$ be the topological space obtained from a family $\left\{\Delta_{\sigma}\right\}$ of disjoint euclidean simplexes, $\sigma \in K$, $\operatorname{dim} \Delta_{\sigma}=\operatorname{dim} \sigma$, with the identification induced by the barycentric maps $\alpha: \Delta_{\sigma \alpha} \rightarrow \Delta_{\sigma}$. An algebraic construction is described which assigns to any system $\left\{\pi_{n}\right\}$ a family $\Re\left\{\pi_{n}\right\}$ of css complexes, called type-complexes, and an equivalence relation in $\Omega$. The study of homotopy type of polyhedra and homotopy classes of maps is equivalent with the study of type-complexes and simplicial maps thereof, in consequence of the following facts. 1 . For any space $X$, every minimal complex at $x \in X$ is isomorphic with every member of a uniquely determined equivalence class in $\Omega\left\{\pi_{n}(X, x)\right\}$. 2. For any abstract system $\left\{\pi_{n}\right\}$, any complex $K \in \Omega\left\{\pi_{n}\right\}$ is isomorphic with the minimal complex of the topological space $|K|$, and the system $\left\{\pi_{n}\right\}$ is isomorphic with the system $\left\{\pi_{n}(|K|, e)\right\}, e$ the 0 -simplex of $K$. 3. For any css complex $L$, if $M$ is a minimal complex of $|L|$, then $|M|$ and $|L|$ are of the same homotopy type. 4 . Any continuous map of $|L|$ into $|K|, K \in \Omega$, is homotopic to a simplicial map. (Received July 18, 1955.) 